\title{
Synthesis and Conformational Analysis of Novel Trimeric Maleimide Cross-
} Linking Reagents

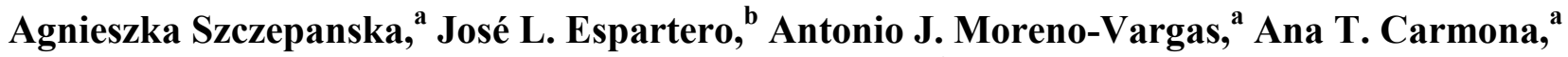 \\ Inmaculada Robina ${ }^{\mathrm{a}}$ * \\ a. Dept. Organic Chemistry, Faculty of Chemistry, University of Seville, P.O. Box 553, E-41071 Seville, SPAIN. E- \\ mail:robina@us.es. \\ b. Dept. Organic and Pharmaceutical Chemistry, Faculty of Pharmacy, University of Seville, SPAIN.
}

\section{Sarah Remmert and Carol Parish*}

Dept. of Chemistry, University of Richmond, Gottwald Science Center, Richmond, VA, 23173, U.S.A. E-mail: cparish@richmond.edu

\section{SUPPORTING INFORMATION}

Table of contents.

\section{Synthesis and NMR}

Data for compounds: 8(a-c), 9 (a-c), 10(b-c) 4(a-c), 5(a-c), 6(b-c) (Pages S2 - S6)

\section{NMR studies at different emperatures}

NOE contacts for 4a. NOESY experiments (Pages S5- S6)

NOE contacts for 5c. NOESY experiments (Pages S7 - S8)

NOE contacts for $\mathbf{6 c}$. (Pages S9)

${ }^{1} \mathrm{H}-\mathrm{NMR}$ and ${ }^{13} \mathrm{C}-\mathrm{NMR}$ Spectra of compounds 5b, 5c, 6a, 6b, 9b, 9c, 10b (Pages S10 - S16)

\section{Computational data}

Table S1. Force field parameter analysis for Compounds $\mathbf{4 c}, \mathbf{5 c}$ and $\mathbf{6 c}$ (Page S17)

Table S2. LM:MC conformational search results on the OPLS2005/GBSA(water) surface of 4a. (Page S18)

Table S3. LM:MC conformational search results on the OPLS2005/GBSA(water) surface for 5a. (Page S19)

Table S4. LM:MC conformational search results on the OPLS2005/GBSA(water) surface for 6a. (Page S20)

Table S5. LM:MC conformational search results on the OPLS2005/GBSA(water) surface for $\mathbf{4 b .}$ (Page S21)

Table S6. LM:MC conformational search results on the OPLS2005/GBSA(water) surface for $\mathbf{5 b}$. (Page S22)

Table S7. LM:MC conformational search results on the OPLS2005/GBSA(water) surface for $\mathbf{6 b}$. (Page S23)

Table S8. LM:MC conformational search results on the OPLS2005/GBSA(water) surface for 4c. (Page S24)

Table S9. LM:MC conformational search results on the OPLS2005/GBSA(water) surface for 5c. (Page S25)

Table S10. LM:MC conformational search results on the OPLS2005/GBSA(water) surface for 6c. (Page S26 - S27)

Cartesian coordinates for $\mathbf{4 a}$ (Page S28)

Cartesian coordinates for $\mathbf{4 b}$ (Page S29)

Cartesian coordinates for $\mathbf{4 c}$ (Page S31)

Cartesian coordinates for 5a (Page S33)

Cartesian coordinates for $\mathbf{5 b}$ (Page S34)

Cartesian coordinates for 5c (Page S35)

Cartesian coordinates for $\mathbf{6 a}$ (Page S37)

Cartesian coordinates for $\mathbf{6 b}$ (Page S38)

Cartesian coordinates for $\mathbf{6 c}$ (Page S39)

Conformational search details (Page S42 - S44) 
Density functional theory results (methodological treatment, program, input and Cartesian coordinates for 4a (Page S44-S49)

Boc-protected triamine templates 8(a-c), 9(a-c), 10 (a-c).

KTA-( $\boldsymbol{N}$-t-butoxycarbonyl-ethylenediamine $)_{3}$, 8a:See reference 6 , and ${ }^{1} \mathrm{H}$ NMR $\left(300 \mathrm{MHz}, \mathrm{CDCl}_{3}\right)$ : $\delta 1.05(\mathrm{~d}, 3 \mathrm{H}, \mathrm{J}=15.6 \mathrm{~Hz}), 1.21(\mathrm{~s}, 9 \mathrm{H}), 1.43(\mathrm{~s}, 27 \mathrm{H}), 2.86(\mathrm{~d}, 3 \mathrm{H}, \mathrm{J}=15.6 \mathrm{~Hz}), 3.21$ (bs, 12H), 5.84 (bs, $3 \mathrm{H}), 7.43$ (bs, 3H).

KTA-(1-t-Butoxycarbonylamino-3-oxa-pentan-5-amine), 8b: See reference 6 and ${ }^{1} \mathrm{H}$ NMR (300 $\left.\mathrm{MHz}, \mathrm{CDCl}_{3}\right) \delta: 1.07$ (d, 3H, J=15.6Hz), $1.24(\mathrm{~s}, 9 \mathrm{H}), 1.43$ (s, 27H), 2.95 (d, 3H, J=15.3Hz), 3.20-3.41 $(\mathrm{m}, 12 \mathrm{H}), 3.47(\mathrm{t}, 6 \mathrm{H}, \mathrm{J}=5.1 \mathrm{~Hz}), 3.54(\mathrm{t}, 6 \mathrm{H}, \mathrm{J}=5.1 \mathrm{~Hz}), 6.49(\mathrm{bs}, 3 \mathrm{H}), 7.68(\mathrm{t}, 3 \mathrm{H}, \mathrm{J}=4.3 \mathrm{~Hz})$.

KTA-(1-t-Butoxycarbonylamino-3,6-dioxa-octan-8-amine), 8c: See reference, 6 and ${ }^{1} \mathrm{H}$ NMR (300 $\left.\mathrm{MHz}, \mathrm{CDCl}_{3}\right), \delta 1.05(\mathrm{~d}, 3 \mathrm{H}, \mathrm{J}=15.3 \mathrm{~Hz}), 1.20(\mathrm{~s}, 9 \mathrm{H}), 1.44(\mathrm{~s}, 27 \mathrm{H}), 2.87(\mathrm{~d}, 3 \mathrm{H}, \mathrm{J}=15.6 \mathrm{~Hz}), 3.25-3.38$ $(\mathrm{m}, 12 \mathrm{H}), 3.49(\mathrm{t}, 6 \mathrm{H}, \mathrm{J}=6.3 \mathrm{~Hz}), 3.55(\mathrm{t}, 6 \mathrm{H}, \mathrm{J}=5.1 \mathrm{~Hz}), 3.61(\mathrm{bs}, 12 \mathrm{H}), 5.23(\mathrm{bs}, 3 \mathrm{H}), 7.56(\mathrm{t}, 3 \mathrm{H}$, $\mathrm{J}=5.1 \mathrm{~Hz})$.

Cis,cis-Cyclohexane-1,3,5-tricarboxylic acid-( $N$-t-butoxycarbonyl-ethylenediamine) $)_{3}$, 9a:

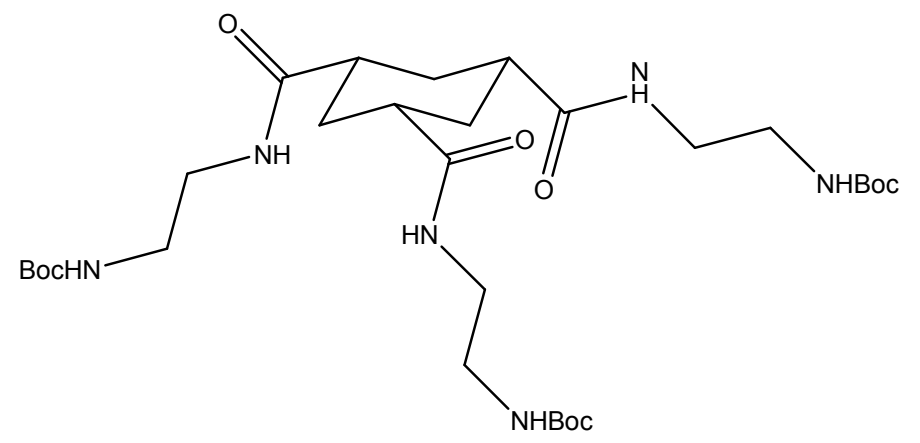

$54 \%$ Yield. White solid (m.p. $200^{\circ} \mathrm{C}$ decomp.). The product proved to have a very poor solubility in most organic solvents. MS (FAB) TIOG. +NaI: 343 (14) $[\mathrm{M}-3 \mathrm{Boc}+\mathrm{H}]+, 365(22)[\mathrm{M}-3 \mathrm{Boc}+\mathrm{Na}]^{+}, 565$ (5) $[\mathrm{M}-\mathrm{Boc}+\mathrm{Na}]^{+}, 665$ (68) $\quad[\mathrm{M}+\mathrm{Na}]^{+}, 707$ (100) $\quad[\mathrm{M}+\mathrm{Na}]^{+}$. HRMS (FAB): calc. $[\mathrm{M}+\mathrm{Na}]^{+}$ $\mathrm{C}_{30} \mathrm{H}_{54} \mathrm{~N}_{6} \mathrm{O}_{9} \mathrm{Na}$ 665.3850. Found: 665.3872.

Cis,cis-Cyclohexane-1,3,5-tricarboxylic acid-(1-t-Butoxycarbonylamino-3-oxa-pentan-5-amine), 9b:

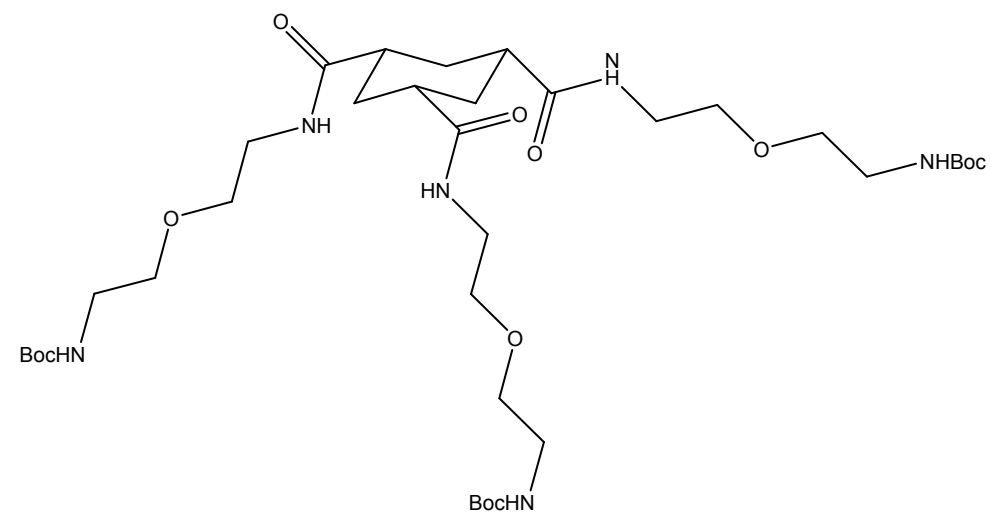

29\% Yield. White solid (m.p.181-183 ${ }^{\circ} \mathrm{C}$ ). ${ }^{1} \mathrm{H}$ NMR $\left(\mathrm{CD}_{3} \mathrm{OD}, 300 \mathrm{MHz}\right) \delta: 1.45$ (s, 27H), 1.63 (dd, $\left.3 \mathrm{H}, J_{A B}=12.6 \mathrm{~Hz}\right), 1.92(\mathrm{bd}, 3 \mathrm{H}, J=12.6 \mathrm{~Hz}), 2.38(\mathrm{tt}, J=12.6 \mathrm{~Hz}, J=3.3 \mathrm{~Hz}, 3 \mathrm{H}), 3.22(\mathrm{t}, 6 \mathrm{H}, J=5.7 \mathrm{~Hz})$, $3.36(\mathrm{t}, 6 \mathrm{H}, J=5.7 \mathrm{~Hz}), 3.49(\mathrm{t}, 6 \mathrm{H}, J=5.7 \mathrm{~Hz}), 3.52(\mathrm{t}, 6 \mathrm{H}, J=5.4 \mathrm{~Hz}), 4.62(\mathrm{bs}, 6 \mathrm{H}) .{ }^{13} \mathrm{C} \mathrm{NMR}\left(\mathrm{CD}_{3} \mathrm{OD}\right.$, $75 \mathrm{MHz}) \delta: 28.9,32.9,40.4,41.4,44.9,70.5,71.0,80.3,158.7,177.6$. MS (FAB) TIOG. +NaI: 475 
(24) $[\mathrm{M}-3 \mathrm{Boc}+\mathrm{H}]^{+}, 497(10)[\mathrm{M}-3 \mathrm{Boc}+\mathrm{Na}]^{+}, 597 \quad$ (5) $[\mathrm{M}-2 \mathrm{Boc}+\mathrm{Na}]^{+}, 675$ (5) $[\mathrm{M}-\mathrm{Boc}+\mathrm{Na}]^{+}, 797$ (100) $[\mathrm{M}+\mathrm{Na}]^{+}$. HRMS (FAB): calc. $[\mathrm{M}+\mathrm{Na}]^{+} \mathrm{C}_{36} \mathrm{H}_{66} \mathrm{~N}_{6} \mathrm{O}_{12} \mathrm{Na}$ 797.4636. Found: 797.4630.

Cis,cis-Cyclohexane-1,3,5-tricarboxylic acid -(1-t-Butoxycarbonylamino-3,6-dioxa-octan-8amine) $)_{3}, 9 \mathrm{c}$

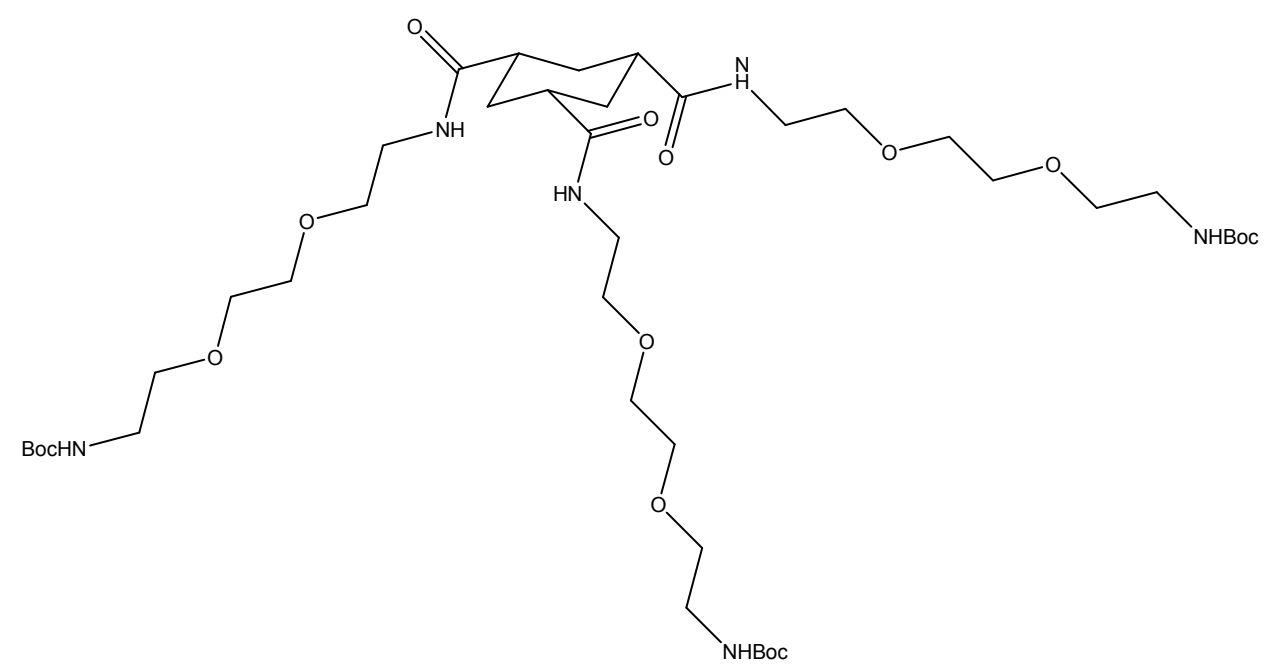

$49 \%$ Yield. White solid (m.p. $\left.129-131^{\circ} \mathrm{C}\right) .{ }^{1} \mathrm{H}$ NMR $\left(\mathrm{CDCl}_{3}, 300 \mathrm{MHz}\right) \delta: 1.44(\mathrm{~s}, 27 \mathrm{H}), 1.62(\mathrm{dd}, 3 \mathrm{H}$, $\left.J_{A B}=12.6 \mathrm{~Hz}\right), 2.09(\mathrm{~d}, 3 \mathrm{H}, J=12.6 \mathrm{~Hz}), 2.25(\mathrm{t}, 3 \mathrm{H}, J=11.7 \mathrm{~Hz}), 3.25-3.36(\mathrm{~m}, 6 \mathrm{H}), 3.40-3.49(\mathrm{~m}, 6 \mathrm{H})$, 3.50-3.65 (m, 24H), 5.19 (bs, 3H), 6.28 (bs, 3H). ${ }^{13} \mathrm{C} \mathrm{NMR}\left(\mathrm{CDCl}_{3}, 75 \mathrm{MHz}\right)$ 8: 28.4, 31.7, 39.2, 40.3, 44.0, 69.7, 70.1, 79.3, 156.1, 174.2. MS (FAB) TIOG. +NaI: 607 (7) $[\mathrm{M}-3 \mathrm{Boc}+\mathrm{H}]^{+}, 929$ (25) $[\mathrm{M}+\mathrm{Na}]^{+}$. HRMS (FAB): calc. $[\mathrm{M}+\mathrm{Na}]^{+} \mathrm{C}_{42} \mathrm{H}_{78} \mathrm{~N}_{6} \mathrm{O}_{15} \mathrm{Na} 929.5423$. Found: 929.5427.

Trimesic acid-(1-t-Butoxycarbonylamino-3-oxa-pentan-5-amine) ${ }_{3}$, 10b:<smiles></smiles>

37\% Yield. Colorless oil. ${ }^{1} \mathrm{H}$ NMR $\left(\mathrm{CDCl}_{3}, 300 \mathrm{MHz}\right) \delta: 1.38(\mathrm{~s}, 27 \mathrm{H}), 3.20-3.40(\mathrm{bm}, 6 \mathrm{H}), 3.57(\mathrm{t}$, $6 \mathrm{H}, J=5.1 \mathrm{~Hz}), 3.66$ (bs, 12H), 5.18 (bs, 3H), 7.35 (bs, 3H), 8.36 (s, 3H). ${ }^{13} \mathrm{C} \mathrm{NMR}\left(\mathrm{CDCl}_{3}, 75 \mathrm{MHz}\right)$ $\delta: 28.3,40.0,40.4,69.4,70.2,79.4,128.5,134.9,156.3,166.2$. MS (FAB) TIOG. +NaI: 469 (100) $[\mathrm{M}-3 \mathrm{Boc}+\mathrm{H}]^{+}, 491(57)[\mathrm{M}-3 \mathrm{Boc}+\mathrm{Na}]^{+}, 591(15)[\mathrm{M}-2 \mathrm{Boc}+\mathrm{Na}]^{+}, 791$ (73) $[\mathrm{M}+\mathrm{Na}]^{+}$. HRMS (FAB): calc. $[\mathrm{M}+\mathrm{Na}]^{+} \mathrm{C}_{36} \mathrm{H}_{60} \mathrm{~N}_{6} \mathrm{O}_{12} \mathrm{Na}$ 791.4167. Found: 791.4166.

Trimesic acid-(1-t-Butoxycarbonylamino-3,6-dioxa-octan-8-amine) $)_{3}$, 10c:

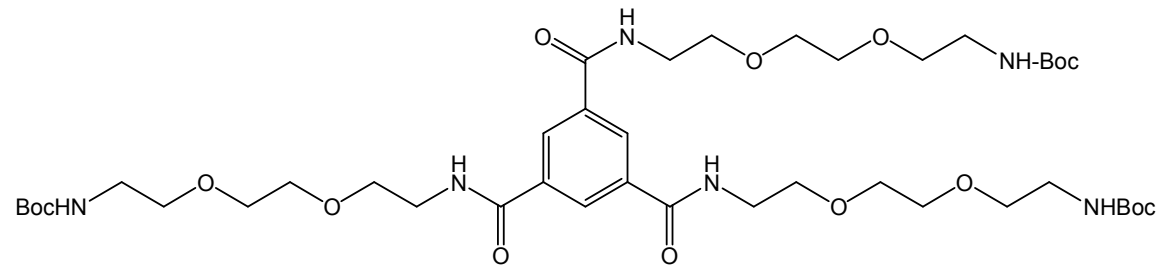

Colorless oil. ${ }^{1} \mathrm{H}$ NMR $\left(\mathrm{CDCl}_{3}, 300 \mathrm{MHz}\right) \delta$ : $1.40(\mathrm{~s}, 27 \mathrm{H}), 3.22-3.34(\mathrm{~m}, 6 \mathrm{H}), 3.56(\mathrm{t}, 6 \mathrm{H}, \mathrm{J}=5.4 \mathrm{~Hz})$, 3.60-3.73 (m, 24H), 5.34 (bs, 3H), 7.31 (bs, 3H), 8.45 (s, 3H). ${ }^{13} \mathrm{C} \mathrm{NMR}\left(\mathrm{CDCl}_{3}, 75 \mathrm{MHz}\right) \delta: 28.4$, 
40.0, 40.3, 69.7, 70.1, 70.2, 79.2, 128.6, 135.0, 156.1, 165.9. MS (FAB+) TIOG. +NaI: 601 (100) $[\mathrm{M}-3 \mathrm{Boc}+\mathrm{H}]^{+}, 623(60)[\mathrm{M}-3 \mathrm{Boc}+\mathrm{Na}]^{+}, 723(6)[\mathrm{M}-2 \mathrm{Boc}+\mathrm{Na}]^{+}, 801(5)[\mathrm{M}-\mathrm{Boc}+\mathrm{H}]^{+}, 923(75)$ $[\mathrm{M}+\mathrm{Na}]^{+}$. HRMS (FAB+): calc. $[\mathrm{M}+\mathrm{Na}]^{+} \mathrm{C}_{42} \mathrm{H}_{72} \mathrm{~N}_{6} \mathrm{O}_{15} \mathrm{Na}$ 923.4953. Found: : 923.4961.

KTA-(1-maleimide-ethylenamine) $)_{3}$, 4a: See reference 6 and ${ }^{1} \mathrm{H}$ NMR $\left(300 \mathrm{MHz}, \mathrm{CDCl}_{3}\right), \delta 1.00(\mathrm{~d}$, $3 \mathrm{H}, \mathrm{J}=15.6 \mathrm{~Hz}), 1.13(\mathrm{~s}, 9 \mathrm{H}), 2.73(\mathrm{~d}, 3 \mathrm{H}, \mathrm{J}=15.6 \mathrm{~Hz}), 3.25\left(\mathrm{dd}, 6 \mathrm{H}, \mathrm{J}_{\mathrm{AB}}=5.7 \mathrm{~Hz}\right), 3.60(\mathrm{t}, 6 \mathrm{H}, \mathrm{J}=5.7 \mathrm{~Hz})$, $6.71(\mathrm{~s}, 6 \mathrm{H}), 7.44(\mathrm{t}, 3 \mathrm{H}, \mathrm{J}=5.7 \mathrm{~Hz})$.

Cis,cis-Cyclohexane-1,3,5-tricarboxylic acid 1-maleimide-ethylenamine) $)_{3}$, 5a

98\% Yield. White solid. ${ }^{1} \mathrm{H}$ NMR (DMSO, $\left.300 \mathrm{MHz}\right) \delta: 1.15-1.40$ (m, 3H), 1.57 (d, 3H, $J=11.7 \mathrm{~Hz}$ ), 1.90-2.10 (m, 3H), $3.18(\mathrm{~m}, 6 \mathrm{H}), 3.43(\mathrm{~m}, 6 \mathrm{H}), 6.98(\mathrm{~s}, 6 \mathrm{H}), 7.85(\mathrm{t}, 3 \mathrm{H}, J=5.7 \mathrm{~Hz}) .{ }^{13} \mathrm{C}$ NMR (DMSO, $75 \mathrm{MHz}) \delta: 31.0,36.7,37.2,42.8,134.5,171.0,174.3$. MS (FAB) NITR. +NaI: 605 (24) [M+Na] HRMS (FAB): calc. [M+Na] ${ }^{+} \mathrm{C}_{27} \mathrm{H}_{30} \mathrm{~N}_{6} \mathrm{O}_{9} \mathrm{Na}$ 605.1972. Found: 605.1959.

\section{Trimesic acid-(1-maleimide-ethylenamine) $)_{3}, 6 a$}

White solid (m.p. 177-180 ${ }^{\circ} \mathrm{C}$ ). ${ }^{1} \mathrm{H}$ NMR (DMSO, $\left.300 \mathrm{MHz}\right) \delta: 3.43$ (dd, $6 \mathrm{H}, J_{A B}=5.4 \mathrm{~Hz}$ ), 3.61 (dd, $6 \mathrm{H}, J=5.1 \mathrm{~Hz}, J=5.7 \mathrm{~Hz}), 7.01(\mathrm{~s}, 6 \mathrm{H}), 8.27(\mathrm{~s}, 3 \mathrm{H}), 8.81(\mathrm{t}, 3 \mathrm{H}, J=5.7 \mathrm{~Hz}) .{ }^{13} \mathrm{C}$ NMR (DMSO, $\left.75 \mathrm{MHz}\right)$ $\delta: 37.5,38.2,128.8,135.0,135.2,166.1,171.5$. MS (FAB) NITR. +NaI: $577(8)[\mathrm{M}+\mathrm{H}]^{+}, 599(8)$ $[\mathrm{M}+\mathrm{Na}]^{+}$. HRMS (FAB): calc. $[\mathrm{M}+\mathrm{Na}]^{+} \mathrm{C}_{27} \mathrm{H}_{24} \mathrm{~N}_{6} \mathrm{O}_{9} \mathrm{Na}$ 599.1502. Found: 599.1487 .

KTA-(1-maleimide-3-oxa-pentan-5-amine $)_{3}$, 4b: See reference 6 and ${ }^{1} \mathrm{H}$ NMR $\left(300 \mathrm{MHz}, \mathrm{CDCl}_{3}\right), \delta$ $1.01(\mathrm{~d}, 3 \mathrm{H}, \mathrm{J}=15.6 \mathrm{~Hz}), 1.18(\mathrm{~s}, 9 \mathrm{H}), 2.82(\mathrm{~d}, 3 \mathrm{H}, \mathrm{J}=15.6 \mathrm{~Hz}), 3.20\left(\mathrm{dd}, 6 \mathrm{H}, \mathrm{J}_{\mathrm{AB}}=6.0 \mathrm{~Hz}\right), 3.43(\mathrm{t}, 6 \mathrm{H}$, $\mathrm{J}=6.0 \mathrm{~Hz}), 3.65\left(\mathrm{~A}_{2} \mathrm{~B}_{2}\right.$ system, $\left.12 \mathrm{H}\right), 6.70(\mathrm{~s}, 6 \mathrm{H}), 7.44(\mathrm{t}, 3 \mathrm{H}, \mathrm{J}=5.4 \mathrm{~Hz})$.

\section{Cis,cis-Cyclohexane-1,3,5-tricarboxylic acid-(1-maleimide-3-oxa-pentan-5-amine) $)_{3}, 5 b$}

53\% Yield. White solid (m.p. 200-205 ${ }^{\circ} \mathrm{C}$ ). ${ }^{1} \mathrm{H}$ NMR (DMSO, $\left.300 \mathrm{MHz}\right) \delta: 1.34$ (m, 3H), 1.65 (d, 3H), 2.08-2.22 (m, 3H), $3.12\left(\mathrm{dd}, 6 \mathrm{H}, J_{A B}=5.7 \mathrm{~Hz}\right), 3.36-3.41(\mathrm{~m}, 6 \mathrm{H}), 3.52\left(\mathrm{~A}_{2} \mathrm{~B}_{2}\right.$ system, $\left.12 \mathrm{H}\right), 7.00(\mathrm{~s}$, $6 \mathrm{H}), 7.69$ (t, 3H, $J=5.7 \mathrm{~Hz}) .{ }^{13} \mathrm{C}$ NMR (DMSO, $\left.75 \mathrm{MHz}\right) \delta: 31.5,36.7,38.3,42.6,66.8,68.6,134.5$, 170.9, 174.3. MS (FAB) NITR. +NaI: $715(5)[\mathrm{M}+\mathrm{H}]^{+}, 737$ (8) $[\mathrm{M}+\mathrm{Na}]^{+}$. HRMS (FAB): calc. $[\mathrm{M}+\mathrm{Na}]^{+} \mathrm{C}_{33} \mathrm{H}_{42} \mathrm{~N}_{6} \mathrm{O}_{12} \mathrm{Na}$ 737.2758. Found: 737.2754.

\section{Trimesic acid-(1-maleimide-3-oxa-pentan-5-amine) $)_{3}, 6 \mathrm{~b}$}

61\% Yield. Yellow oil. ${ }^{1} \mathrm{H}$ NMR $\left(\mathrm{CDCl}_{3}, 300 \mathrm{MHz}\right) \delta: 3.58-3.70(\mathrm{~m}, 18 \mathrm{H}), 3.71(\mathrm{~m}, 6 \mathrm{H}), 6.77(\mathrm{~s}, 6 \mathrm{H})$, $7.18(\mathrm{bm}, 3 \mathrm{H}), 8.52(\mathrm{~s}, 3 \mathrm{H}) .{ }^{13} \mathrm{C} \mathrm{NMR}\left(\mathrm{CDCl}_{3}, 75 \mathrm{MHz}\right) \delta: 37.1,40.1,68.3,68.8,128.7,134.3,135.0$, 165.9, 171.2. MS (FAB) NITR. +NaI: $709(5)[\mathrm{M}+\mathrm{H}]^{+}, 731$ (7) $[\mathrm{M}+\mathrm{Na}]^{+}$. HRMS (FAB): calc. $[\mathrm{M}+\mathrm{Na}]^{+} \mathrm{C}_{33} \mathrm{H}_{36} \mathrm{~N}_{6} \mathrm{O}_{12} \mathrm{Na}$ 731.2289. Found: 731.2285.

KTA-(1-maleimide-3,6-dioxa-octan-8-amine) ${ }_{3}$, 4c: See reference 6 and ${ }^{1} \mathrm{H}$ NMR $\left(300 \mathrm{MHz}, \mathrm{CDCl}_{3}\right)$, $\delta 1.04(\mathrm{~d}, 3 \mathrm{H}, \mathrm{J}=15.6 \mathrm{~Hz}), 1.20(\mathrm{~s}, 9 \mathrm{H}), 2.87(\mathrm{~d}, 3 \mathrm{H}, \mathrm{J}=15.3 \mathrm{~Hz}), 3.26\left(\mathrm{dd}, 6 \mathrm{H}, \mathrm{J}_{\mathrm{AB}}=5.7 \mathrm{~Hz}\right), 3.45(\mathrm{t}, 6 \mathrm{H}$, $\mathrm{J}=5.7 \mathrm{~Hz}), 3.58\left(\mathrm{~A}_{2} \mathrm{~B}_{2}\right.$ system, $\left.18 \mathrm{H}\right), 3.69\left(\mathrm{~A}_{2} \mathrm{~B}_{2}\right.$ system, 6H), $6.72(\mathrm{~s}, 6 \mathrm{H}), 7.52(\mathrm{t}, 3 \mathrm{H}, \mathrm{J}=5.4 \mathrm{~Hz})$.

Cis,cis-Cyclohexane-1,3,5-tricarboxylic acid -(1-maleimide-3,6-dioxa-octan-8-amine) 3 , 5c

$52 \%$ Yield. White solid (m.p. $\left.120-123^{\circ} \mathrm{C}\right) .{ }^{1} \mathrm{H}$ NMR $\left(\mathrm{CDCl}_{3}, 300 \mathrm{MHz}\right) \delta: 1.67\left(\mathrm{dd}, 3 \mathrm{H}, J_{A B}=12.6 \mathrm{~Hz}\right)$, $2.12(\mathrm{~d}, 3 \mathrm{H}, J=12.9 \mathrm{~Hz}), 2.34(\mathrm{t}, 3 \mathrm{H}, J=12.3 \mathrm{~Hz}), 3.35-3.44(\mathrm{~m}, 6 \mathrm{H}), 3.45-3.61(\mathrm{~m}, 18 \mathrm{H}), 3.68\left(\mathrm{~A}_{2} \mathrm{~B}_{2}\right.$ system, $12 \mathrm{H}), 6.32(\mathrm{t}, 3 \mathrm{H}, J=5.4 \mathrm{~Hz}), 6.76(\mathrm{~s}, 6 \mathrm{H}) .{ }^{13} \mathrm{C} \mathrm{NMR}\left(\mathrm{CDCl}_{3}, 75 \mathrm{MHz}\right) \delta: 31.7,37.2,39.1$, 44.1, 67.9, 69.8, 69.9, 70.2, 134.2, 170.8, 174.3. MS (FAB) NITR. +NaI: 847 (6) [M+Na] $]^{+}, 869$ (20) $[\mathrm{M}+\mathrm{Na}]^{+}$. HRMS (FAB): calc. $[\mathrm{M}+\mathrm{Na}]^{+} \mathrm{C}_{39} \mathrm{H}_{54} \mathrm{~N}_{6} \mathrm{O}_{15} \mathrm{Na} 869.3545$. Found: 869.3575 .

Cis,cis-Cyclohexane-1,3,5-tricarboxylic acid-(1-maleimide-3,6-dioxa-octan-8-amine), 6c: See reference 6 and ${ }^{1} \mathrm{H}$ NMR $\left(300 \mathrm{MHz}, \mathrm{CDCl}_{3}\right), \delta 3.55-3.76(\mathrm{~m}, 36 \mathrm{H}), 6.68(\mathrm{~s}, 6 \mathrm{H}), 7.26(\mathrm{bm}, 3 \mathrm{H}), 8.47$ (s, 3H). ${ }^{13} \mathrm{C}$ NMR $\left(75 \mathrm{MHz}, \mathrm{CDCl}_{3}\right), \delta 37.1,40.1,67.9,69.7,70.0,70.2,128.5,134.1,135.1,166.0$, 170.7. FABMS (NBA): $841(5)[\mathrm{M}+\mathrm{H}]^{+}, 863(24)[\mathrm{M}+\mathrm{Na}]^{+}$. HRFABMS calcd. $[\mathrm{M}+\mathrm{Na}]^{+}$ $\mathrm{C}_{39} \mathrm{H}_{48} \mathrm{~N}_{6} \mathrm{O}_{15} \mathrm{Na}$ 863.3075; Found: 863.3099. 
NMR studies at different emperatures

NOE contacts for $\mathbf{4 a}$.

NOESY experiments
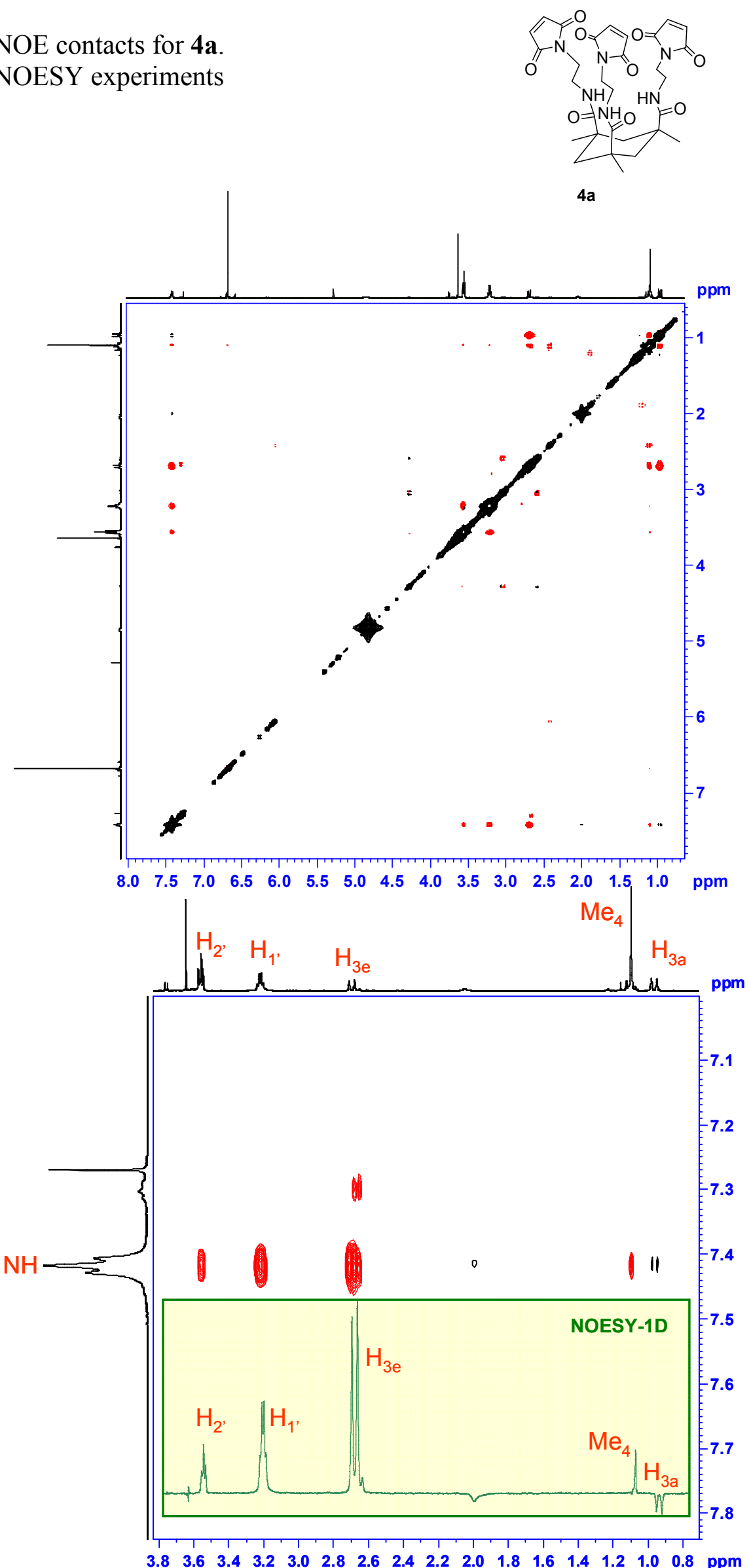


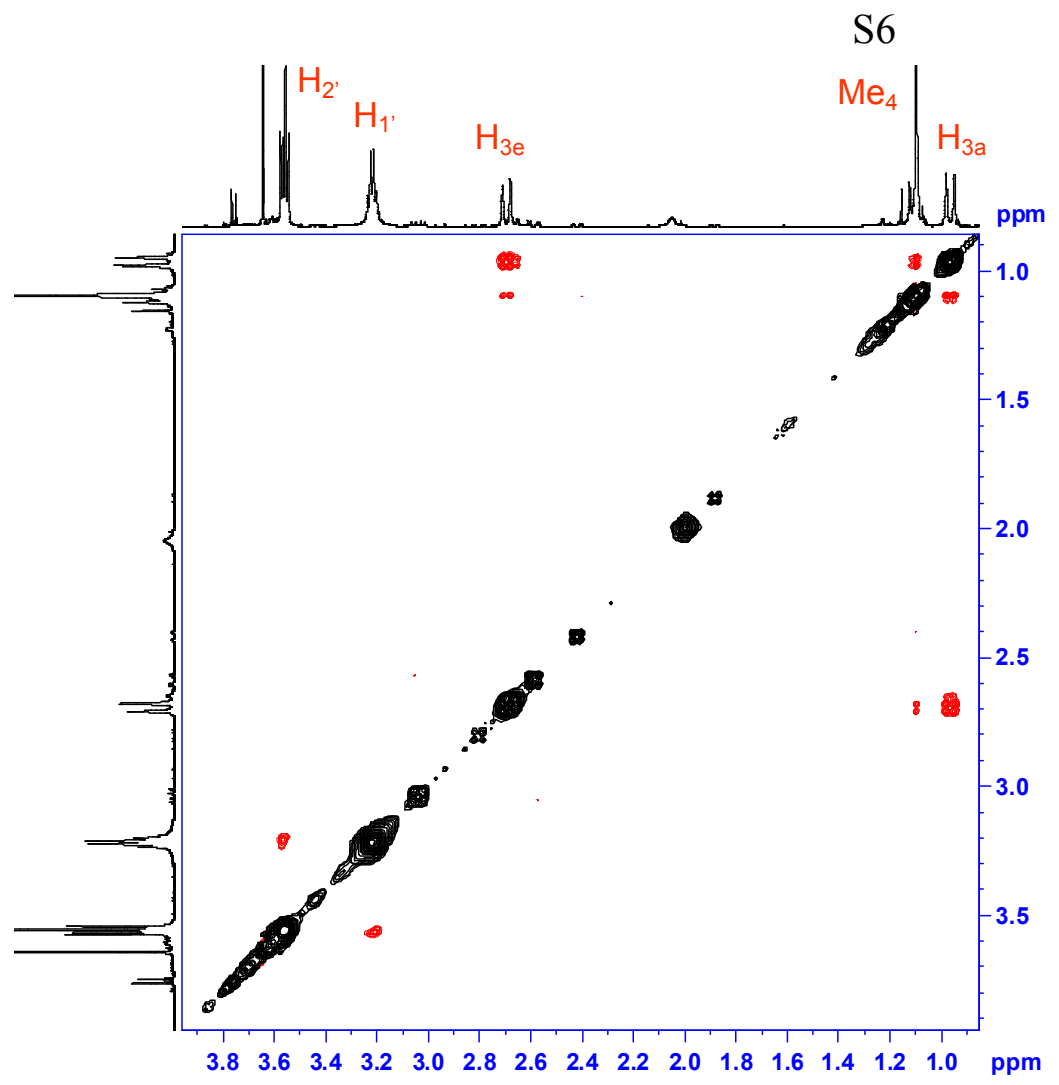


NOE contacts for $\mathbf{5 c}$.

NOESY experiments
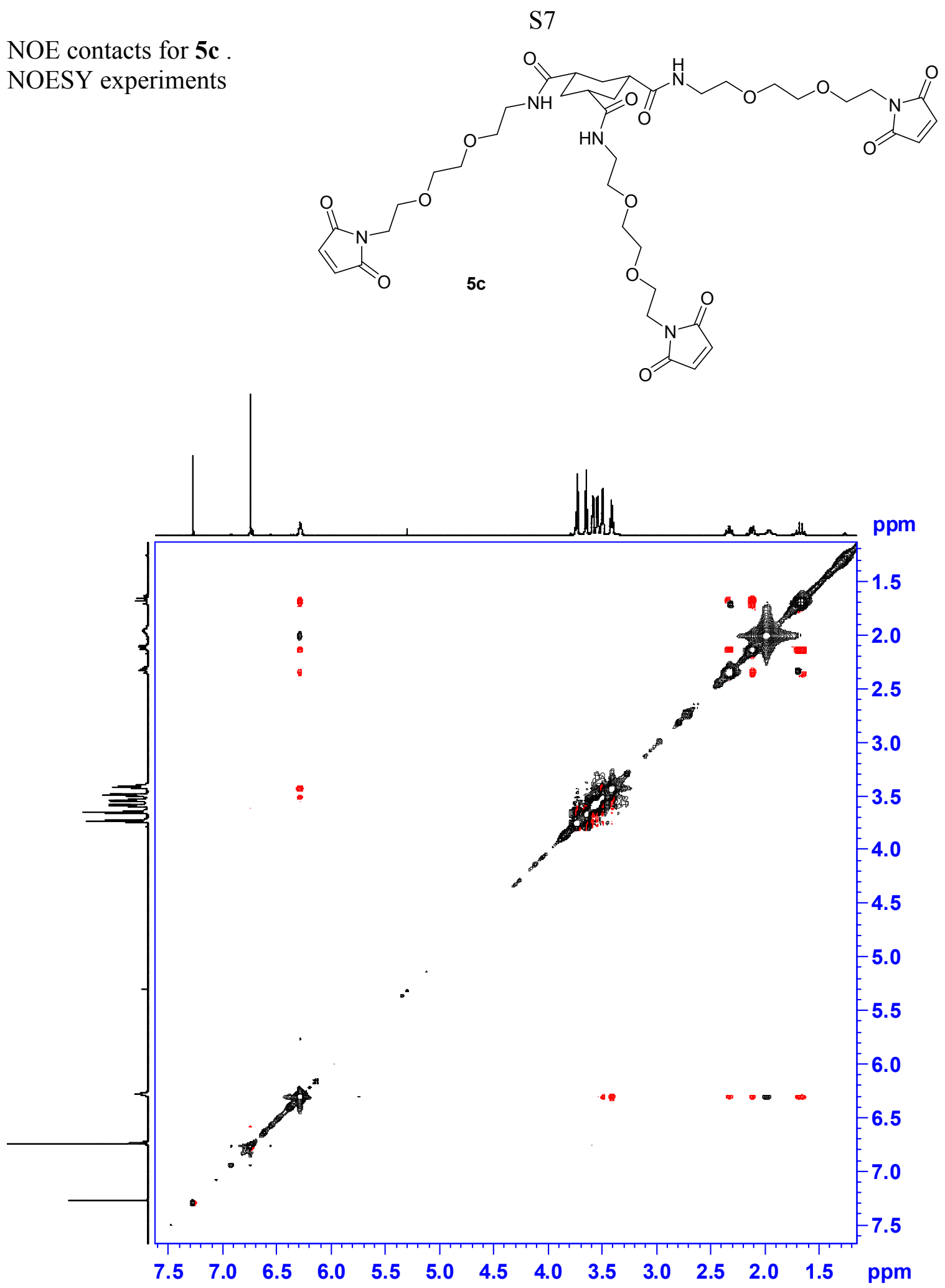

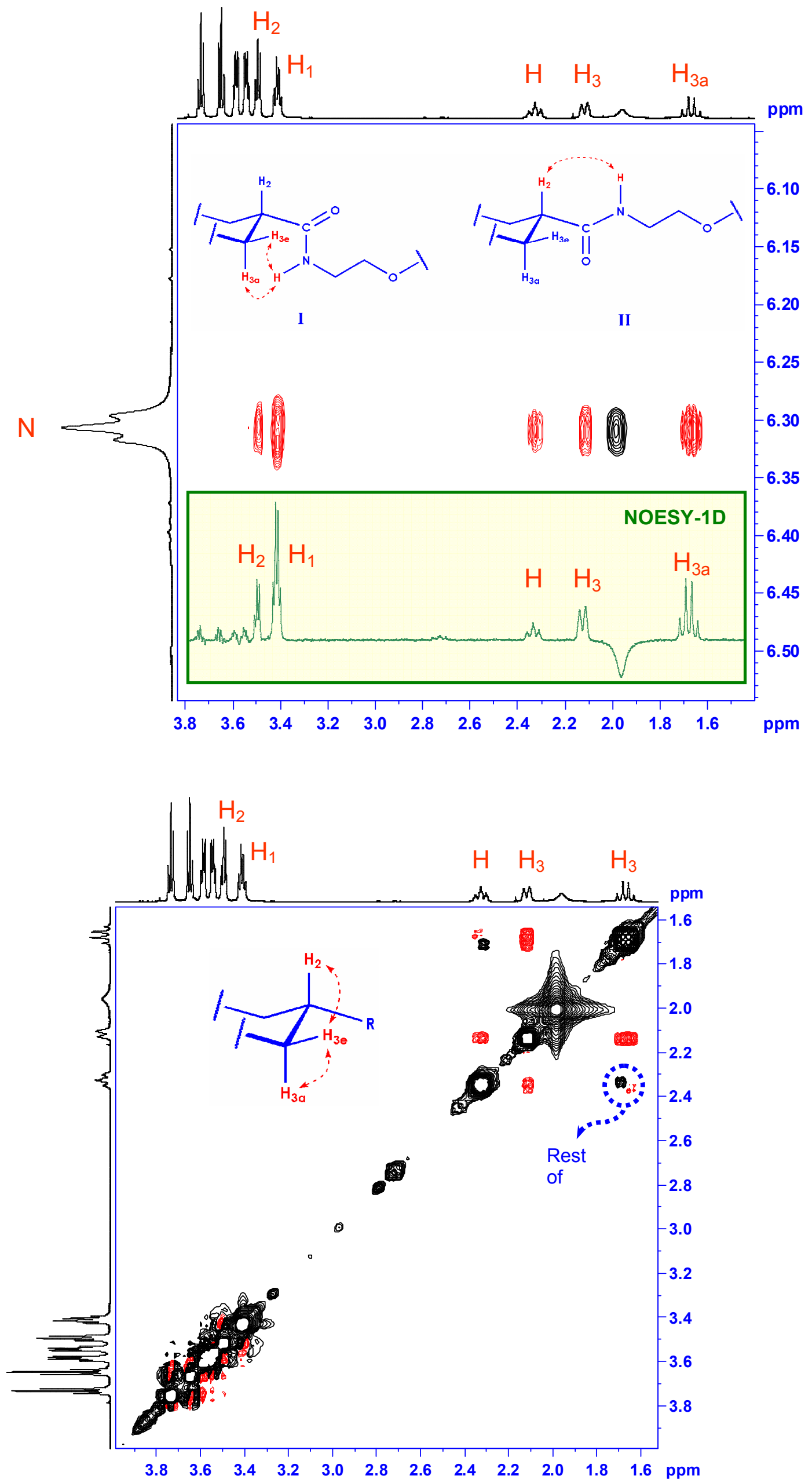
NOE contacts for $\mathbf{6 c}$

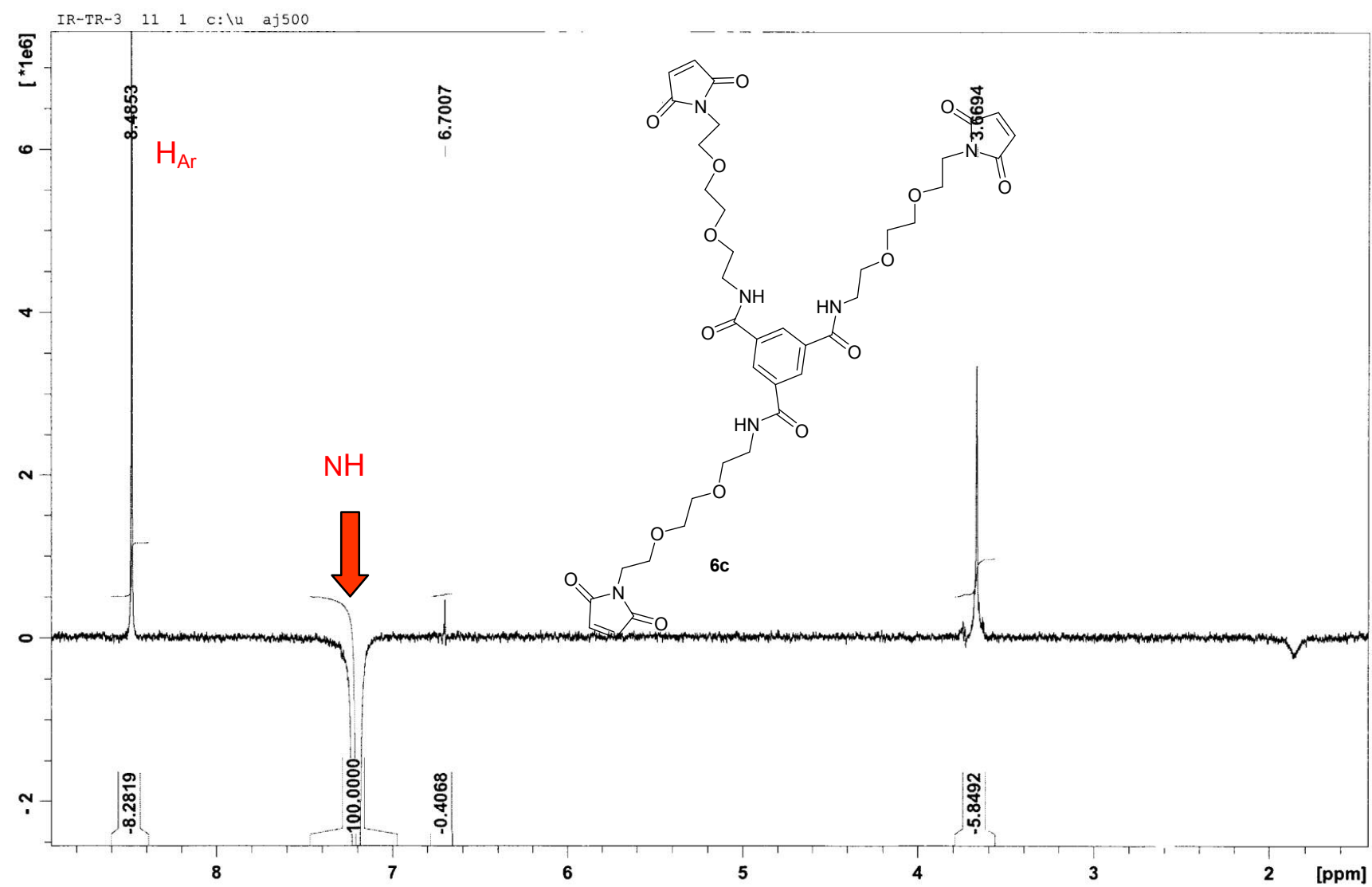


Spectra for compounds: 5b, 5c, 6a, 6b, 9b, 9c, $10 b$
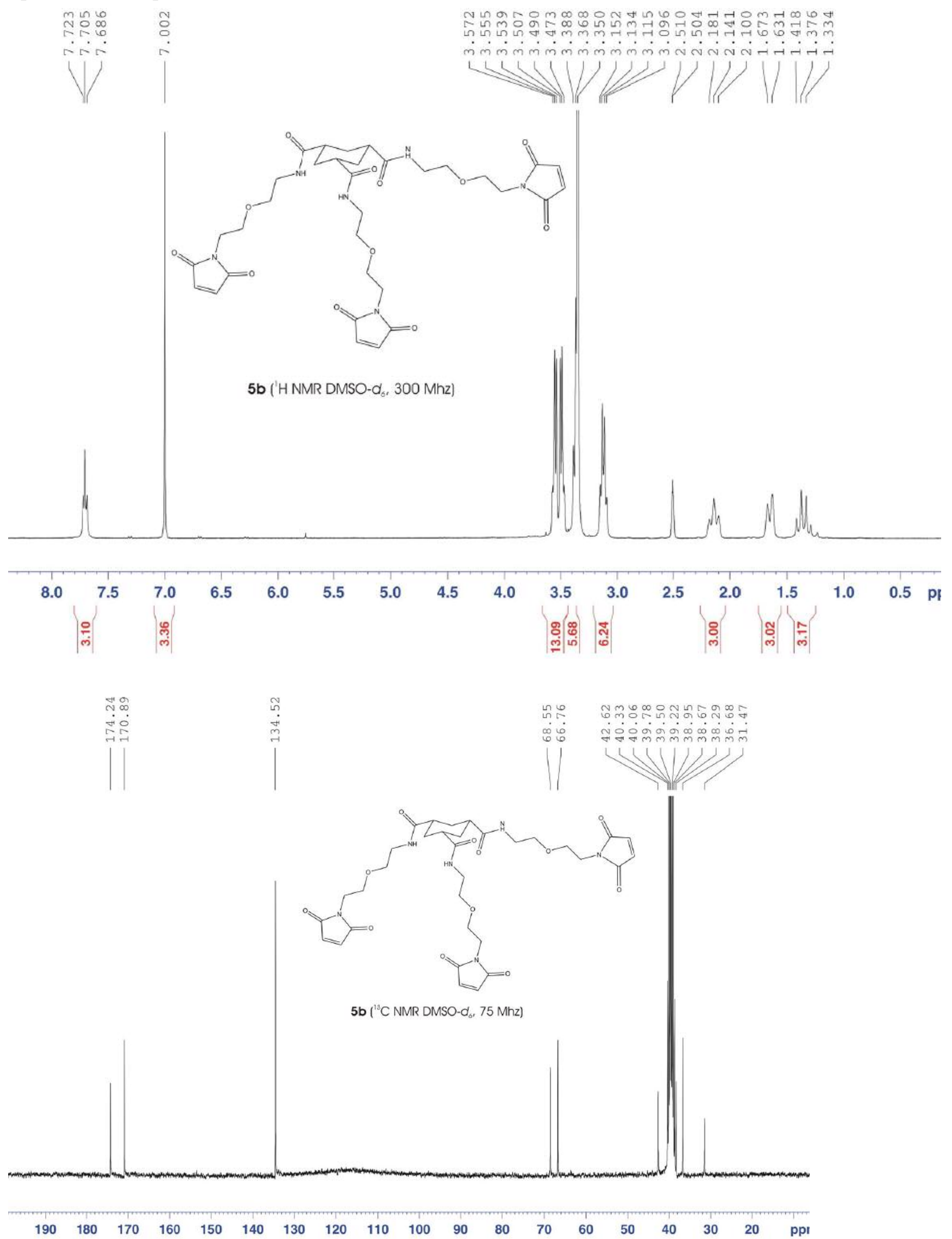

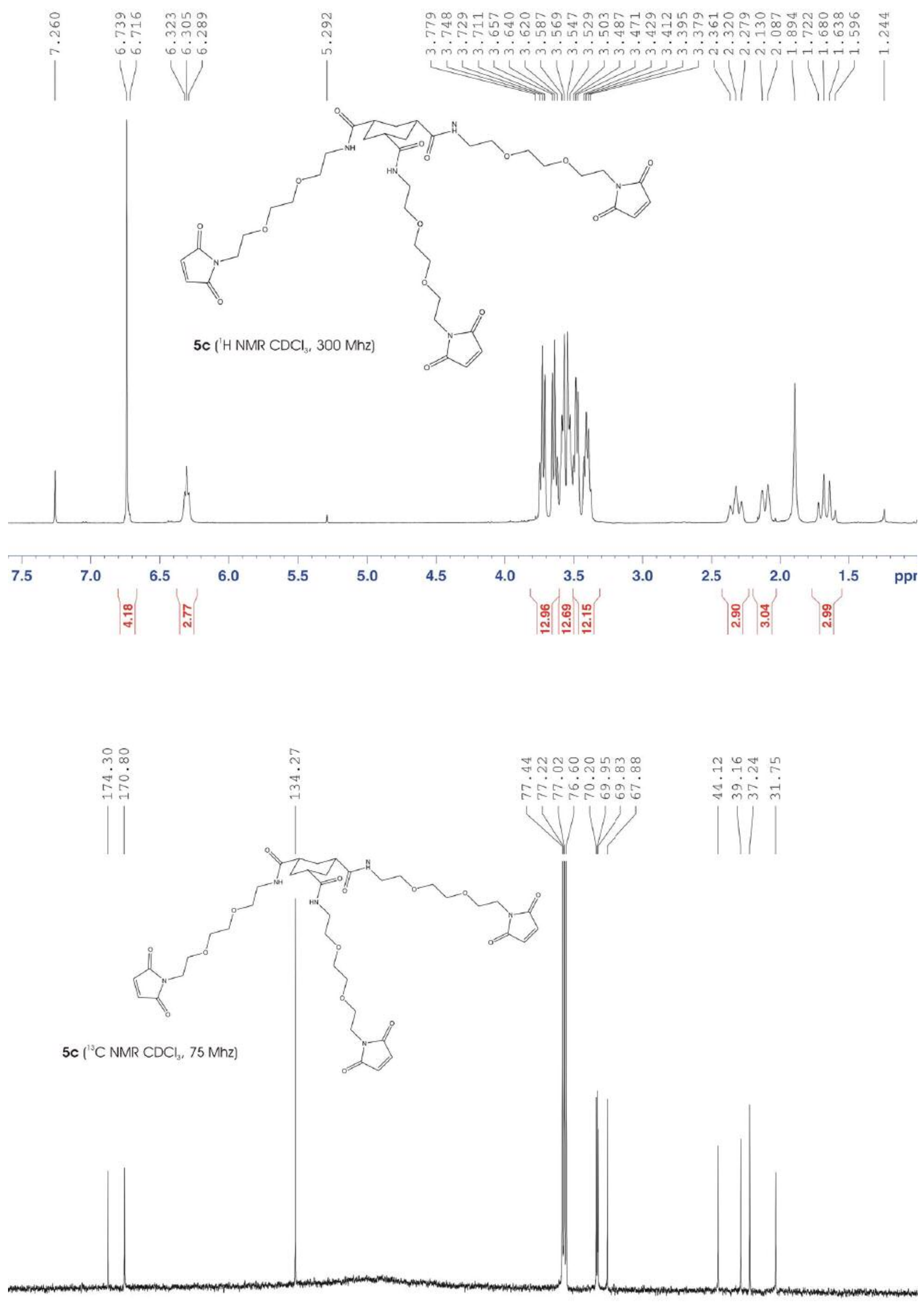

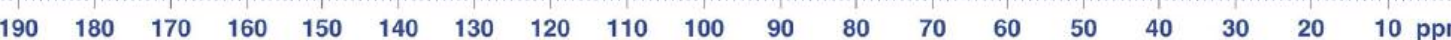



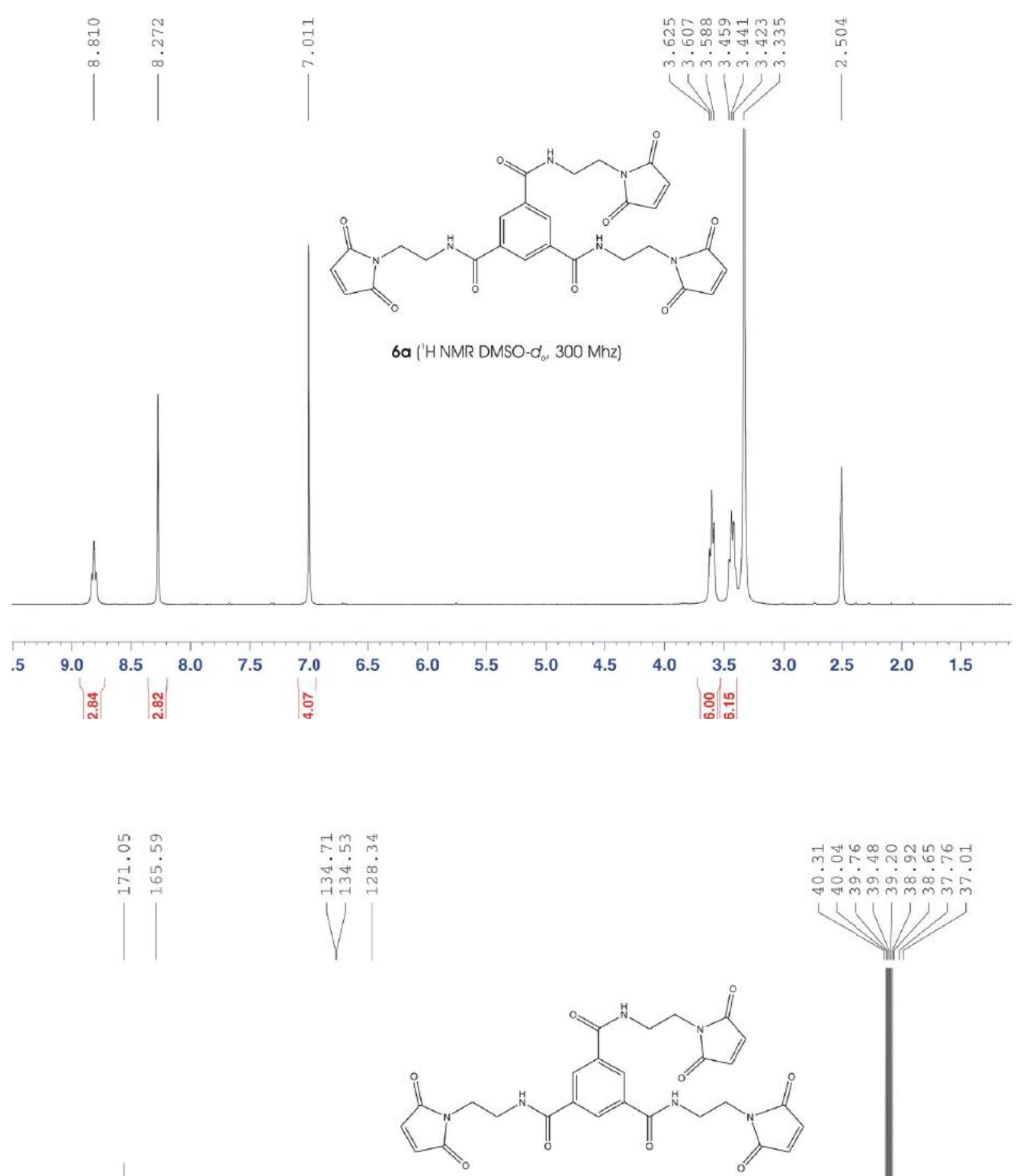

6a ( ${ }^{13} \mathrm{C}$ NMR DMSO- $\left.d_{6,}, 75 \mathrm{Mhz}\right)$

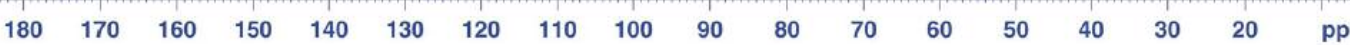




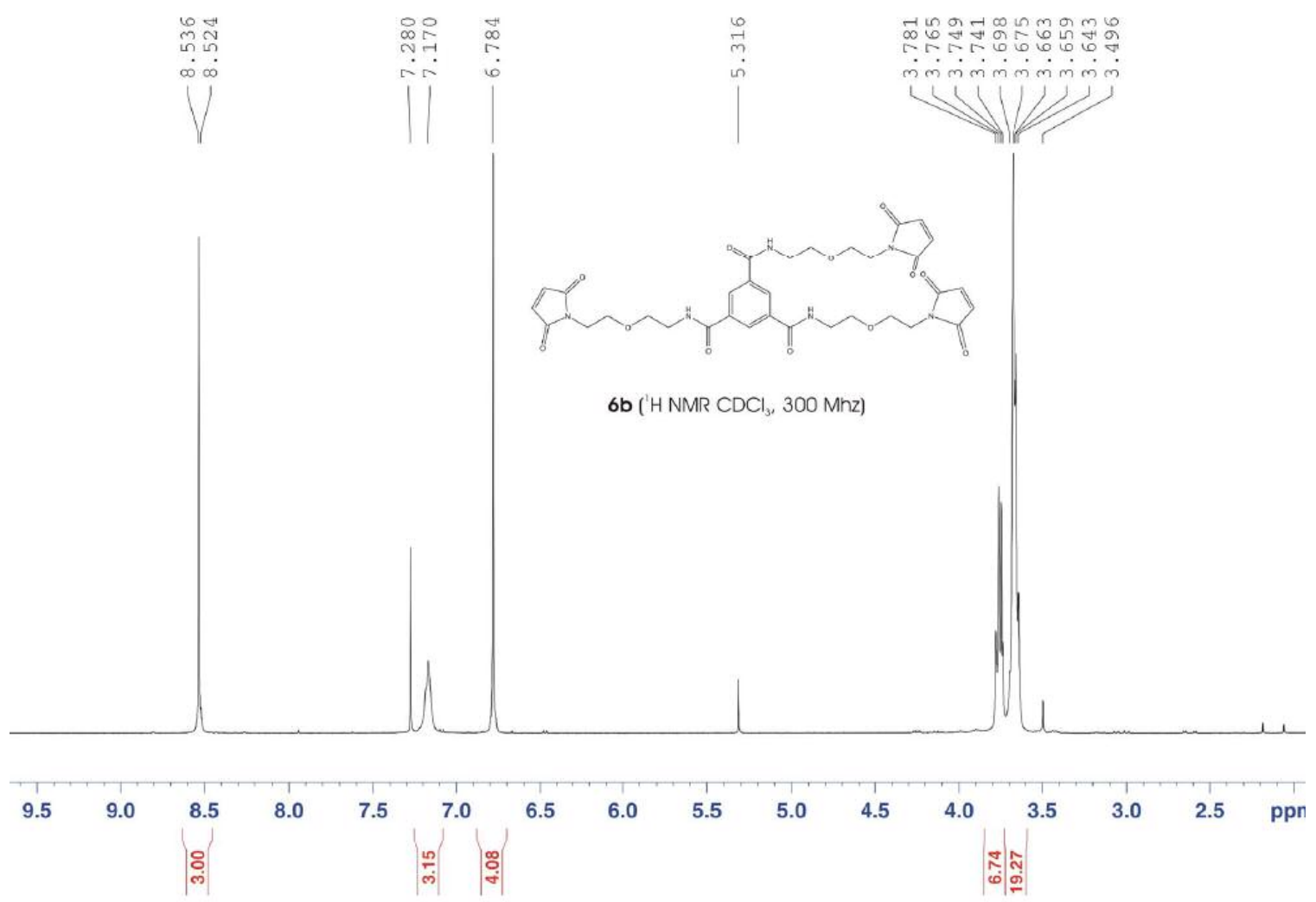

กั

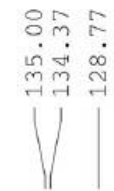

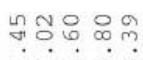

금

है

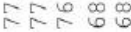

VV

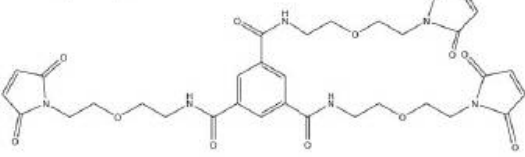

6b ( $\left({ }^{13} \mathrm{C} \mathrm{NMR} \mathrm{CDCl} 3,75 \mathrm{Mhz}\right)$

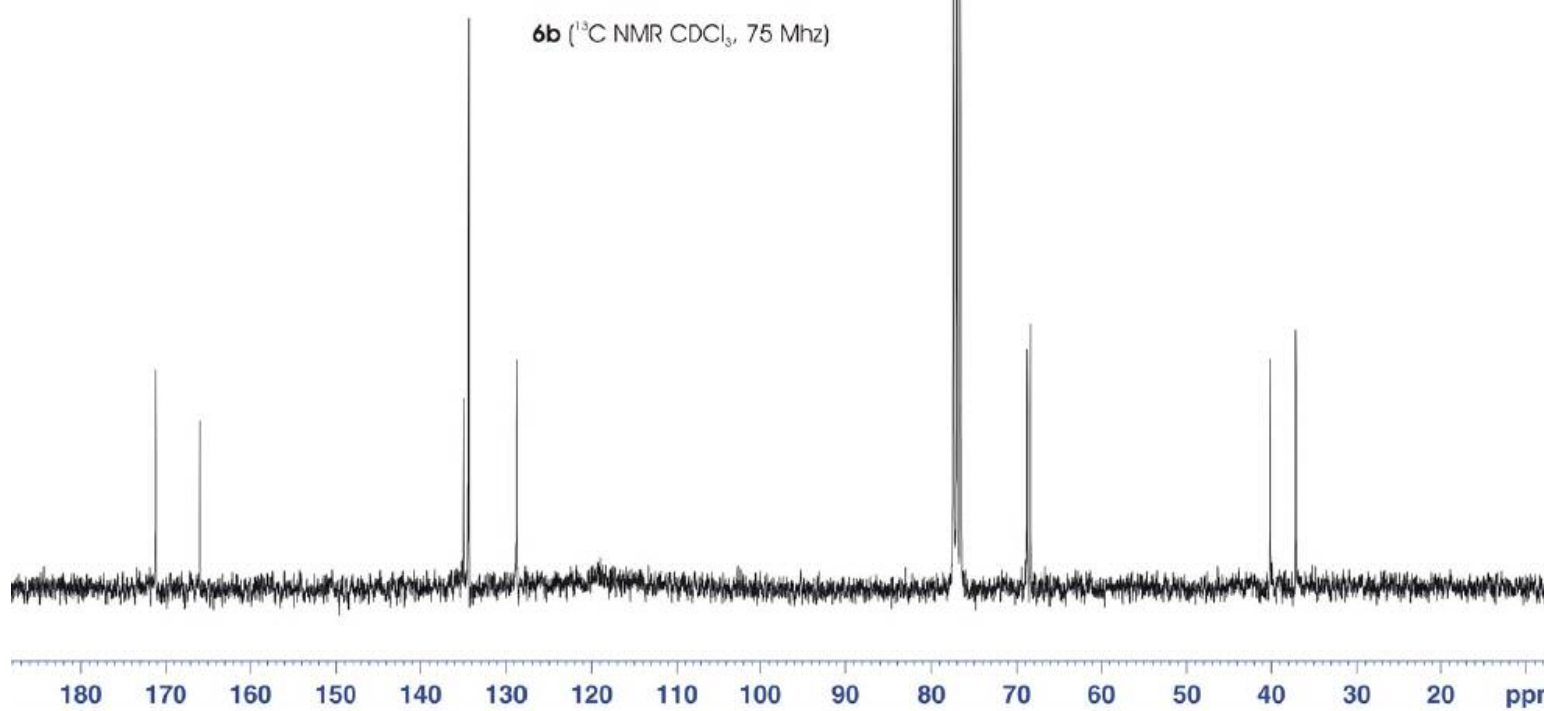



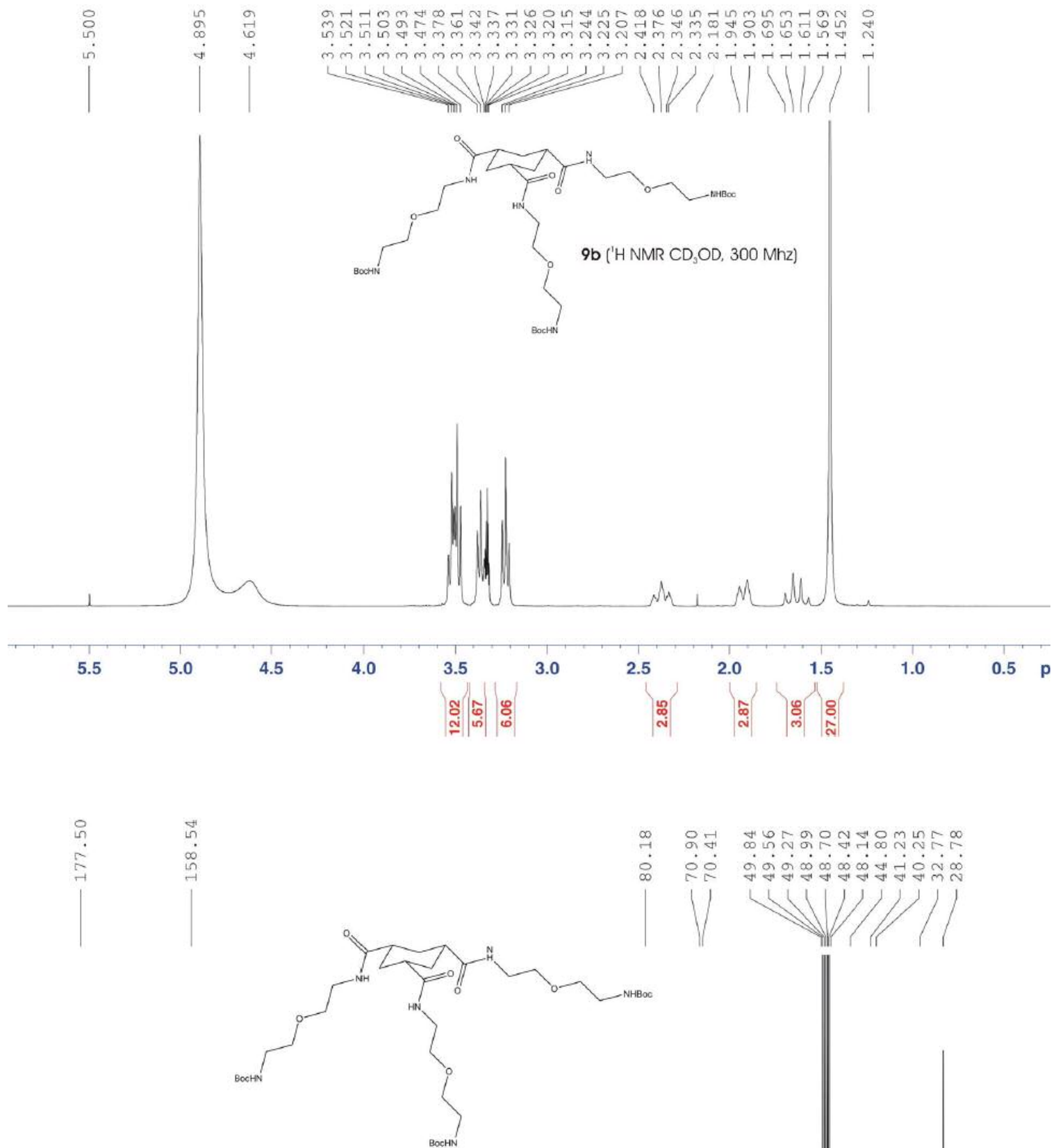

9b $\left({ }^{13} \mathrm{C}\right.$ NMR $\left.\mathrm{CD}_{3} \mathrm{OD}, 75 \mathrm{Mhz}\right)$

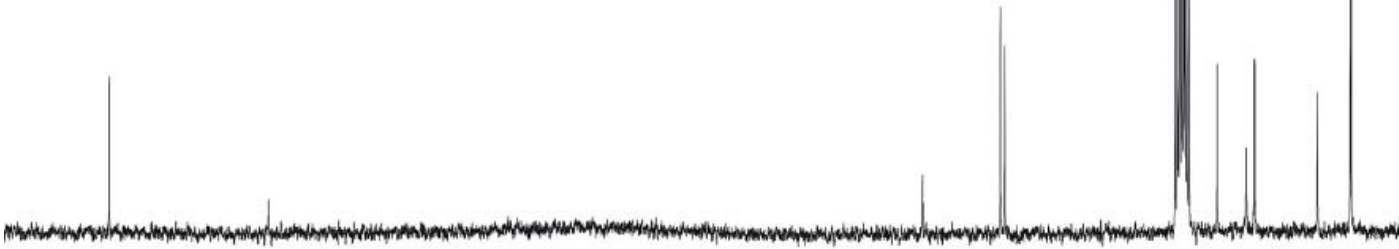

$\begin{array}{llllllllllllllllll}180 & 170 & 160 & 150 & 140 & 130 & 120 & 110 & 100 & 90 & 80 & 70 & 60 & 50 & 40 & 30 & 20 & \mathrm{ppr}\end{array}$ 

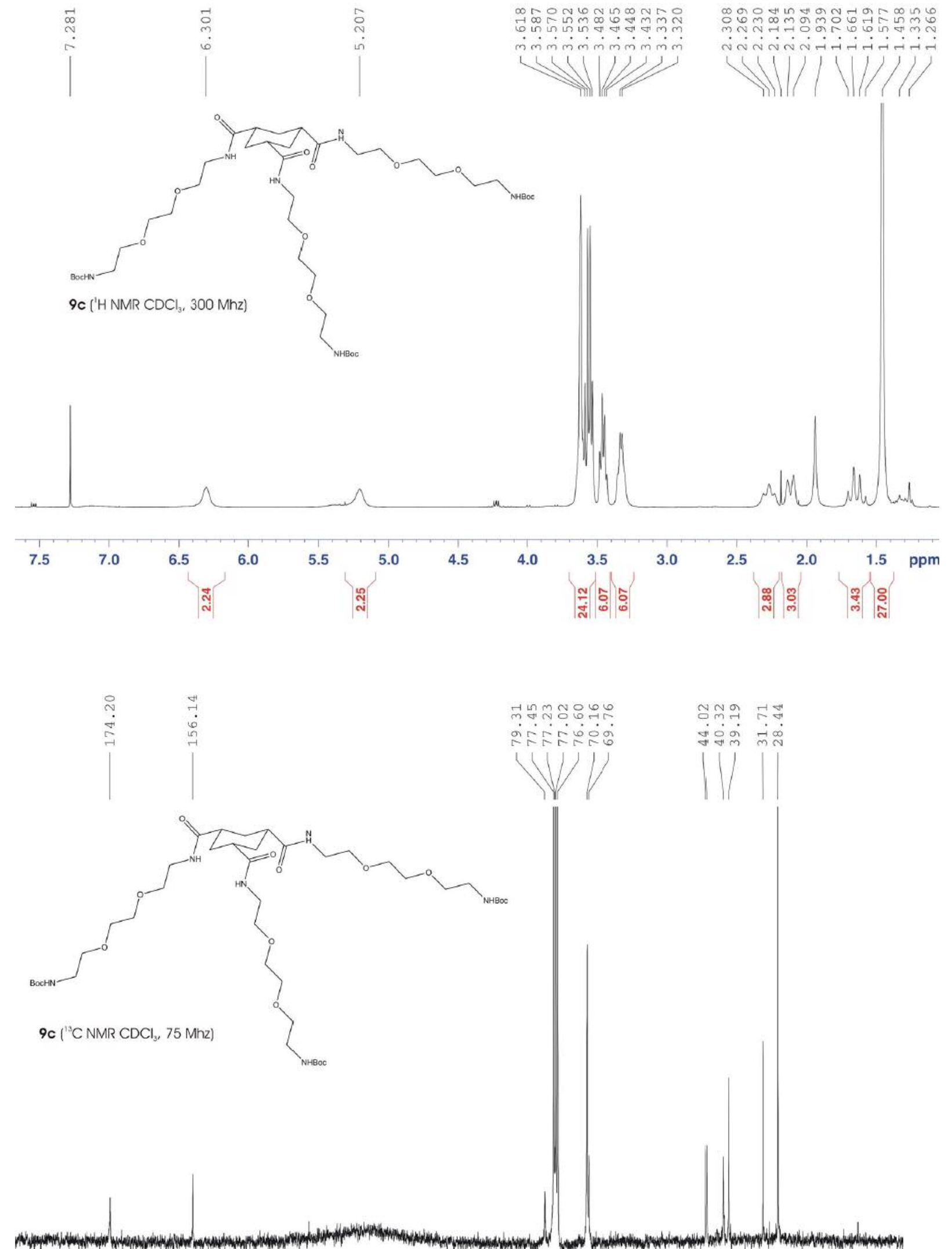

$9 \mathrm{c}\left({ }^{13} \mathrm{C} \mathrm{NMR} \mathrm{CDCl}_{3}, 75 \mathrm{Mhz}\right.$

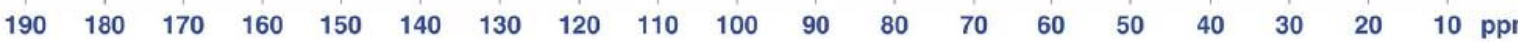



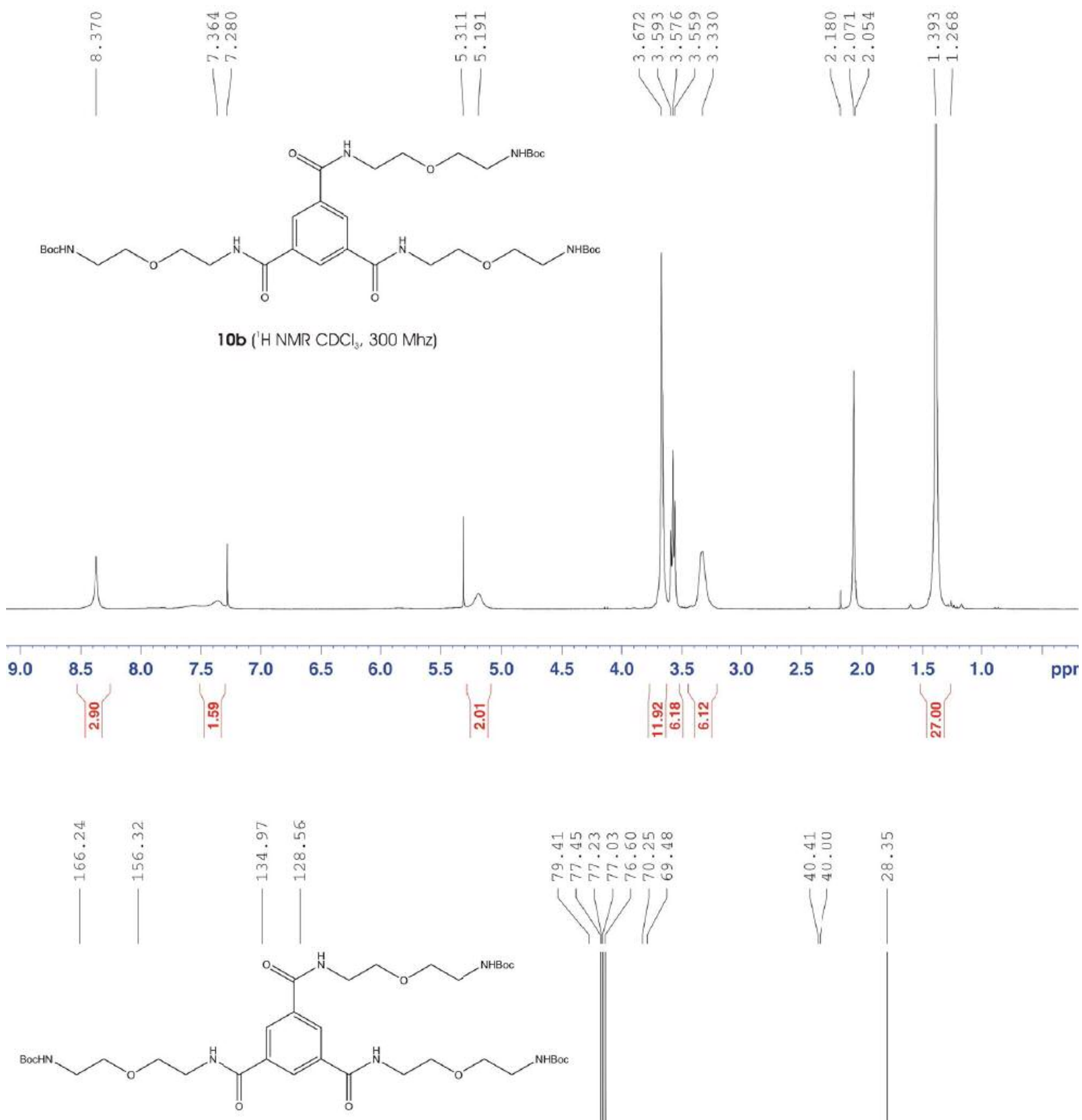

$10 b\left({ }^{13} \mathrm{C}\right.$ NMR $\left.\mathrm{CDCl}_{3}, 75 \mathrm{Mhz}\right)$

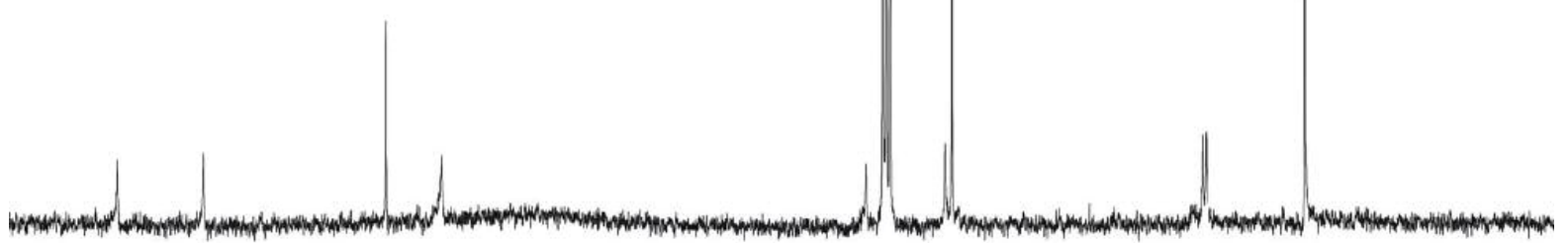

$\begin{array}{llllllllllllllllll}170 & 160 & 150 & 140 & 130 & 120 & 110 & 100 & 90 & 80 & 70 & 60 & 50 & 40 & 30 & 20 & 10 & \mathrm{ppm}\end{array}$ 


\section{Computational data}

Table S1. Force field parameter analysis for Compounds $\mathbf{4 c}, \mathbf{5 c}$ and $\mathbf{6 c}$

$\mathrm{H}=$ high quality parameter, $\mathrm{M}=$ medium quality parameter, $\mathrm{L}=$ low quality parameter.

\begin{tabular}{|c|c|c|c|c|c|c|c|c|c|c|}
\hline \multirow[t]{2}{*}{ Force Field } & \multirow{2}{*}{$\begin{array}{c}\text { Interaction } \\
\text { Type }\end{array}$} & \multicolumn{3}{|c|}{ Compound $4 c$} & \multicolumn{3}{|c|}{ Compound 5c } & \multicolumn{3}{|c|}{ Compound $\mathbf{6 c}$} \\
\hline & & $\mathrm{H}$ & M & $\mathrm{L}$ & $\mathrm{H}$ & $\mathrm{M}$ & $\mathrm{L}$ & $\mathrm{H}$ & M & $\mathrm{L}$ \\
\hline \multirow{3}{*}{$\begin{array}{l}\text { AMBER* } \\
\text { all-atom }\end{array}$} & stretch & 74 & 6 & 3 & 70 & 6 & 3 & 60 & 9 & 3 \\
\hline & bend & 198 & 12 & 0 & 180 & 12 & 0 & 153 & 21 & 0 \\
\hline & torsion & 156 & 73 & 0 & 132 & 74 & 0 & 75 & 75 & 0 \\
\hline \multirow[t]{3}{*}{ MMFF } & stretch & 126 & 0 & 0 & 117 & 0 & 0 & 111 & 0 & 0 \\
\hline & bend & 231 & 0 & 0 & 213 & 0 & 0 & 192 & 0 & 3 \\
\hline & torsion & 303 & 0 & 21 & 276 & 0 & 21 & 228 & 0 & 33 \\
\hline \multirow[t]{3}{*}{ MM2* } & stretch & 105 & 27 & 0 & 96 & 27 & 0 & 81 & 27 & 0 \\
\hline & bend & 189 & 63 & 0 & 171 & 63 & 0 & 138 & 63 & 0 \\
\hline & torsion & 192 & 177 & 21 & 165 & 177 & 21 & 102 & 165 & 24 \\
\hline \multirow[t]{3}{*}{ MM3* } & stretch & 60 & 12 & 33 & 54 & 12 & 30 & 48 & 9 & 24 \\
\hline & bend & 150 & 54 & 0 & 135 & 51 & 0 & 105 & 45 & 0 \\
\hline & torsion & 189 & 63 & 6 & 162 & 63 & 6 & 111 & 48 & 12 \\
\hline \multirow[t]{3}{*}{ OPLS } & stretch & 84 & 0 & 9 & 75 & 0 & 9 & 60 & 3 & 9 \\
\hline & bend & 216 & 0 & 0 & 198 & 0 & 0 & 177 & 3 & 0 \\
\hline & torsion & 174 & 63 & 0 & 153 & 57 & 0 & 93 & 51 & 6 \\
\hline \multirow[t]{3}{*}{ OPLS2001 } & stretch & 126 & 0 & 0 & 117 & 0 & 0 & 111 & 0 & 0 \\
\hline & bend & 231 & 0 & 0 & 213 & 0 & 0 & 195 & 0 & 0 \\
\hline & torsion & 303 & 0 & 0 & 297 & 0 & 0 & 261 & 0 & 0 \\
\hline \multirow[t]{3}{*}{ OPLS2005 } & stretch & 126 & 0 & 0 & 117 & 0 & 0 & 111 & 0 & 0 \\
\hline & bend & 231 & 0 & 0 & 213 & 0 & 0 & 195 & 0 & 0 \\
\hline & torsion & 318 & 6 & 0 & 291 & 6 & 0 & 255 & 6 & 0 \\
\hline
\end{tabular}


Table S2. LM:MC conformational search results on the OPLS2005/GBSA(water) surface of 4a.

\begin{tabular}{|c|c|c|c|c|c|c|}
\hline $\begin{array}{l}\text { Number } \\
\text { of Steps }\end{array}$ & $\begin{array}{c}\text { Number of } \\
\text { Conformations } \\
\text { Found }\end{array}$ & $\begin{array}{c}\text { Number of } \\
\text { Conformations } \\
\text { Minimized }\end{array}$ & $\begin{array}{c}\text { Number } \\
\text { of Global } \\
\text { Minimum } \\
\text { Visits }\end{array}$ & $\begin{array}{c}\text { Minimum } \\
\text { Energy } \\
(\mathrm{kJ} / \mathrm{Mol})\end{array}$ & $\begin{array}{c}\text { CPU } \\
\text { Time } \\
\text { (Hours) }\end{array}$ & $\begin{array}{l}\text { Number of } \\
\text { Conformations } \\
\text { Found Per } \\
\text { Hour }\end{array}$ \\
\hline 5000 & 12 & 12 & 93 & -517.16 & 1.30 & 9 \\
\hline 5000 & 12 & 12 & 196 & -517.15 & 1.30 & 9 \\
\hline 5000 & 12 & 11 & 289 & -517.15 & 1.30 & 9 \\
\hline 5000 & 13 & 13 & 393 & -517.15 & 1.28 & 10 \\
\hline 5000 & 12 & 12 & 495 & -517.14 & 1.30 & 9 \\
\hline 5000 & 13 & 13 & 583 & -517.14 & 1.28 & 10 \\
\hline 5000 & 13 & 13 & 688 & -517.14 & 1.30 & 10 \\
\hline 5000 & 12 & 12 & 766 & -517.16 & 1.30 & 9 \\
\hline 5000 & 12 & 12 & 848 & -517.15 & 1.30 & 9 \\
\hline 5000 & 12 & 12 & 932 & -517.15 & 1.30 & 9 \\
\hline 5000 & 13 & 13 & 1026 & -517.15 & 1.30 & 10 \\
\hline 5000 & 12 & 12 & 1099 & -517.15 & 1.30 & 9 \\
\hline 5000 & 12 & 12 & 1168 & -517.15 & 1.30 & 9 \\
\hline 5000 & 12 & 12 & 1245 & -517.15 & 1.32 & 9 \\
\hline 5000 & 12 & 12 & 1336 & -517.15 & 1.30 & 9 \\
\hline 5000 & 13 & 13 & 1408 & -517.14 & 1.32 & 10 \\
\hline 5000 & 12 & 12 & 1408 & -517.11 & 0.00 & \\
\hline
\end{tabular}

* Within a $16.736 \mathrm{~kJ} / \mathrm{mol}$ energetic window above the lowest energy structure. 
S19

Table S3. LM:MC conformational search results on the OPLS2005/GBSA(water) surface for 5a.

\begin{tabular}{|c|c|c|c|c|c|c|}
\hline $\begin{array}{l}\text { Number } \\
\text { of Steps }\end{array}$ & $\begin{array}{c}\text { Number of } \\
\text { Conformations } \\
\text { Found }\end{array}$ & $\begin{array}{l}\text { Number of } \\
\text { Conformations } \\
\text { Minimized }\end{array}$ & $\begin{array}{l}\text { Number of } \\
\text { Global } \\
\text { Minimum } \\
\text { Visits }\end{array}$ & $\begin{array}{l}\text { Minimum } \\
\text { Energy } \\
(\mathrm{kJ} / \mathrm{Mol})\end{array}$ & $\begin{array}{c}\text { CPU } \\
\text { Time } \\
\text { (Hours) }\end{array}$ & $\begin{array}{c}\text { Number of } \\
\text { Conformations } \\
\text { Found Per } \\
\text { Hour }\end{array}$ \\
\hline 5000 & 191 & 190 & 32 & -529.02 & 1.15 & 166 \\
\hline 5000 & 203 & 202 & 72 & -529.02 & 1.13 & 179 \\
\hline 5000 & 219 & 219 & 106 & -529.00 & 1.15 & 190 \\
\hline 5000 & 214 & 214 & 140 & -529.01 & 1.13 & 189 \\
\hline 5000 & 211 & 211 & 177 & -529.03 & 1.12 & 189 \\
\hline 5000 & 270 & 270 & 202 & -529.01 & 1.15 & 235 \\
\hline 5000 & 298 & 297 & 222 & -529.02 & 1.15 & 259 \\
\hline 5000 & 312 & 310 & 249 & -529.01 & 1.15 & 271 \\
\hline 5000 & 315 & 311 & 268 & -529.03 & 1.15 & 274 \\
\hline 5000 & 300 & 300 & 295 & -529.02 & 1.15 & 261 \\
\hline 5000 & 312 & 309 & 318 & -529.01 & 1.18 & 264 \\
\hline 5000 & 308 & 307 & 333 & -529.02 & 1.15 & 268 \\
\hline 5000 & 310 & 308 & 363 & -529.01 & 1.13 & 274 \\
\hline 5000 & 308 & 307 & 381 & -529.02 & 1.18 & 260 \\
\hline 5000 & 322 & 320 & 408 & -529.00 & 1.13 & 284 \\
\hline 5000 & 321 & 320 & 436 & -529.01 & 1.17 & 275 \\
\hline 5000 & 313 & 311 & 454 & -529.01 & 1.13 & 276 \\
\hline 5000 & 312 & 311 & 477 & -529.01 & 1.17 & 267 \\
\hline 5000 & 309 & 307 & 494 & -528.99 & 1.12 & 277 \\
\hline 5000 & 317 & 315 & 513 & -528.99 & 1.17 & 272 \\
\hline 5000 & 315 & 315 & 527 & -529.02 & 1.15 & 274 \\
\hline 5000 & 305 & 304 & 544 & -529.01 & 1.13 & 269 \\
\hline 5000 & 306 & 305 & 574 & -529.01 & 1.18 & 259 \\
\hline 5000 & 297 & 296 & 598 & -529.01 & 1.17 & 255 \\
\hline 5000 & 310 & 309 & 619 & -529.00 & 1.17 & 266 \\
\hline 5000 & 307 & 306 & 643 & -529.01 & 1.17 & 263 \\
\hline 5000 & 315 & 315 & 658 & -529.01 & 1.17 & 270 \\
\hline 5000 & 307 & 306 & 677 & -529.01 & 1.13 & 271 \\
\hline 5000 & 318 & 317 & 699 & -529.01 & 1.12 & 285 \\
\hline 5000 & 312 & 312 & 715 & -529.01 & 1.18 & 264 \\
\hline 5000 & 310 & 306 & 731 & -529.00 & 1.15 & 270 \\
\hline 5000 & 322 & 321 & 745 & -528.99 & 1.12 & 288 \\
\hline 5000 & 323 & 320 & 761 & -529.00 & 1.15 & 281 \\
\hline 5000 & 309 & 306 & 782 & -529.00 & 1.15 & 269 \\
\hline 5000 & 303 & 302 & 800 & -529.01 & 1.17 & 260 \\
\hline mini & 271 & 271 & 800 & -528.99 & & \\
\hline
\end{tabular}

\footnotetext{
* Within a $16.736 \mathrm{~kJ} / \mathrm{mol}$ energetic window above the lowest energy structure.
} 
Table S4. LM:MC conformational search results on the OPLS2005/GBSA(water) surface for 6a.

\begin{tabular}{|c|c|c|c|c|c|c|}
\hline $\begin{array}{l}\text { Number } \\
\text { of Steps }\end{array}$ & $\begin{array}{c}\text { Number of } \\
\text { Conformations } \\
\text { Found }\end{array}$ & $\begin{array}{c}\text { Number of } \\
\text { Conformations } \\
\text { Minimized }\end{array}$ & $\begin{array}{l}\text { Number of } \\
\text { Global } \\
\text { Minimum } \\
\text { Visits }\end{array}$ & $\begin{array}{c}\text { Minimum } \\
\text { Energy } \\
(\mathrm{kJ} / \mathrm{Mol})\end{array}$ & $\begin{array}{c}\text { CPU } \\
\text { Time } \\
\text { (Hours) }\end{array}$ & $\begin{array}{c}\text { Number of } \\
\text { Conformations } \\
\text { Found Per } \\
\text { Hour }\end{array}$ \\
\hline 5000 & $2340 *$ & 2340 & 3 & -482.45 & 1.03 & 2265 \\
\hline 5000 & 3280 & 3279 & 4 & -482.57 & 1.45 & 2262 \\
\hline 5000 & 3763 & 3762 & 4 & -482.58 & 1.68 & 2235 \\
\hline 5000 & 4032 & 4028 & 7 & -482.59 & 1.83 & 2199 \\
\hline 5000 & 4210 & 4210 & 8 & -482.59 & 1.92 & 2197 \\
\hline 5000 & 4313 & 4313 & 9 & -482.61 & 1.98 & 2175 \\
\hline 5000 & 4408 & 4404 & 13 & -482.59 & 2.02 & 2186 \\
\hline 5000 & 4441 & 4437 & 16 & -482.60 & 2.05 & 2166 \\
\hline 5000 & 4477 & 4473 & 16 & -482.56 & 2.07 & 2166 \\
\hline 5000 & 4510 & 4506 & 18 & -482.59 & 2.10 & 2148 \\
\hline 5000 & 4531 & 4525 & 18 & -482.56 & 2.12 & 2141 \\
\hline 5000 & $4258^{\dagger}$ & 4254 & 19 & -482.58 & 2.05 & 2077 \\
\hline 5000 & 4265 & 4261 & 21 & -482.56 & 2.05 & 2080 \\
\hline 5000 & 4273 & 4273 & 21 & -482.56 & 2.03 & 2101 \\
\hline 5000 & 4293 & 4292 & 21 & -482.56 & 2.07 & 2077 \\
\hline 5000 & 4305 & 4304 & 25 & -482.56 & 2.07 & 2083 \\
\hline 5000 & 4308 & 4307 & 28 & -482.56 & 2.07 & 2085 \\
\hline 5000 & 4306 & 4305 & 31 & -482.56 & 2.07 & 2084 \\
\hline 5000 & 4301 & 4298 & 33 & -428.57 & 2.07 & 2081 \\
\hline 5000 & 4305 & 4300 & 36 & -428.59 & 2.08 & 2066 \\
\hline 5000 & 4320 & 4320 & 39 & -482.59 & 2.07 & 2090 \\
\hline 5000 & 2570 & 2568 & 41 & -482.58 & 1.52 & 1695 \\
\hline 5000 & 2576 & 2576 & 44 & -482.58 & 1.50 & 1717 \\
\hline 5000 & 2580 & 2579 & 45 & -482.58 & 1.50 & 1720 \\
\hline 5000 & 2583 & 2583 & 46 & -482.56 & 1.48 & 1741 \\
\hline 5000 & 2582 & 2581 & 49 & -482.55 & 1.50 & 1721 \\
\hline 5000 & 2576 & 2576 & 51 & -482.59 & 1.50 & 1717 \\
\hline 5000 & 2576 & 2576 & 55 & -482.59 & 1.48 & 1737 \\
\hline 5000 & 2572 & 2571 & 59 & -482.57 & 1.48 & 1734 \\
\hline 5000 & 2574 & 2573 & 60 & -482.57 & 1.50 & 1716 \\
\hline 5000 & 2575 & 2574 & 63 & -482.57 & 1.48 & 1736 \\
\hline 5000 & 2582 & 2582 & 66 & -482.57 & 1.48 & 1741 \\
\hline 5000 & 2581 & 2581 & 66 & -482.57 & 1.50 & 1721 \\
\hline 5000 & 2574 & 2574 & 67 & -482.57 & 1.48 & 1735 \\
\hline 5000 & 2578 & 2578 & 72 & -482.57 & 1.52 & 1700 \\
\hline $\operatorname{mini}$ & 2563 & 2563 & 72 & -482.57 & & \\
\hline
\end{tabular}

Within a $* 50 \mathrm{~kJ} / \mathrm{mol},{ }^{\dagger} 25 \mathrm{~kJ} / \mathrm{mol}$, or ${ }^{\ddagger} 16.736 \mathrm{~kJ} / \mathrm{mol}$ energetic window above the lowest energy structure 
Table S5. LM:MC conformational search results on the OPLS2005/GBSA(water) surface for $\mathbf{4 b .}$

\begin{tabular}{|c|c|c|c|c|c|c|}
\hline $\begin{array}{l}\text { Number } \\
\text { of Steps }\end{array}$ & $\begin{array}{c}\text { Number of } \\
\text { Conformations } \\
\text { Found }\end{array}$ & $\begin{array}{c}\text { Number of } \\
\text { Conformations } \\
\text { Minimized }\end{array}$ & $\begin{array}{l}\text { Number of } \\
\text { Global } \\
\text { Minimum } \\
\text { Visits }\end{array}$ & $\begin{array}{c}\text { Minimum } \\
\text { Energy } \\
(\mathrm{kJ} / \mathrm{Mol})\end{array}$ & $\begin{array}{c}\text { CPU } \\
\text { Time } \\
\text { (Hours) }\end{array}$ & $\begin{array}{c}\text { Number of } \\
\text { Conformations } \\
\text { Found Per } \\
\text { Hour }\end{array}$ \\
\hline 5000 & 143 & 143 & 35 & -641.23 & 2.12 & 68 \\
\hline 5000 & 220 & 220 & 71 & -641.23 & 2.12 & 104 \\
\hline 5000 & 222 & 222 & 101 & -641.22 & 2.07 & 107 \\
\hline 5000 & 233 & 233 & 137 & -641.23 & 2.07 & 113 \\
\hline 5000 & 233 & 233 & 165 & -641.22 & 2.07 & 113 \\
\hline 5000 & 257 & 257 & 193 & -641.23 & 2.07 & 124 \\
\hline 5000 & 247 & 247 & 223 & -641.23 & 2.07 & 120 \\
\hline 5000 & 249 & 249 & 257 & -641.22 & 2.08 & 120 \\
\hline 5000 & 257 & 257 & 288 & -641.22 & 2.08 & 123 \\
\hline 5000 & 260 & 260 & 332 & -641.22 & 2.10 & 124 \\
\hline 5000 & 253 & 253 & 376 & -641.22 & 2.07 & 122 \\
\hline 5000 & 274 & 274 & 415 & -641.23 & 2.10 & 130 \\
\hline 5000 & 273 & 273 & 450 & -641.22 & 2.08 & 131 \\
\hline 5000 & 278 & 278 & 487 & -641.23 & 2.07 & 135 \\
\hline 5000 & 260 & 260 & 523 & -641.23 & 2.08 & 125 \\
\hline 5000 & 266 & 266 & 563 & -641.23 & 2.07 & 129 \\
\hline 5000 & 274 & 274 & 604 & -641.23 & 2.08 & 132 \\
\hline 5000 & 272 & 272 & 637 & -641.23 & 2.10 & 130 \\
\hline 5000 & 269 & 269 & 665 & -641.23 & 2.08 & 129 \\
\hline 5000 & 274 & 274 & 701 & -641.23 & 2.08 & 132 \\
\hline 5000 & 271 & 271 & 741 & -641.28 & 2.08 & 130 \\
\hline 5000 & 271 & 271 & 777 & -641.27 & 2.10 & 129 \\
\hline 5000 & 271 & 271 & 814 & -641.23 & 2.08 & 130 \\
\hline 5000 & 271 & 271 & 860 & -641.25 & 2.10 & 129 \\
\hline 5000 & 311 & 310 & 897 & -641.23 & 2.12 & 147 \\
\hline 5000 & 281 & 281 & 925 & -641.23 & 2.08 & 135 \\
\hline 5000 & 285 & 285 & 957 & -641.25 & 2.10 & 136 \\
\hline 5000 & 287 & 287 & 993 & -641.23 & 2.08 & 138 \\
\hline 5000 & 281 & 281 & 1022 & -641.23 & 2.08 & 135 \\
\hline 5000 & 287 & 287 & 1062 & -641.23 & 2.10 & 137 \\
\hline 5000 & 291 & 291 & 1091 & -641.23 & 2.08 & 140 \\
\hline 5000 & 283 & 283 & 1120 & -641.22 & 2.08 & 136 \\
\hline 5000 & 281 & 281 & 1151 & -641.23 & 2.08 & 135 \\
\hline 5000 & 287 & 287 & 1186 & -641.23 & 2.12 & 136 \\
\hline 5000 & 292 & 291 & 1207 & -641.23 & 2.10 & 139 \\
\hline mini & 240 & 240 & 1207 & -641.22 & 0.02 & 14400 \\
\hline
\end{tabular}

\footnotetext{
* Within a $16.736 \mathrm{~kJ} / \mathrm{mol}$ energetic window above the lowest energy structure.
} 
Table S6. LM:MC conformational search results on the OPLS2005/GBSA(water) surface for $\mathbf{5 b}$.

\begin{tabular}{|c|c|c|c|c|c|c|}
\hline $\begin{array}{l}\text { Number } \\
\text { of Steps }\end{array}$ & $\begin{array}{c}\text { Number of } \\
\text { Conformations } \\
\text { Found }\end{array}$ & $\begin{array}{c}\text { Number of } \\
\text { Conformations } \\
\text { Minimized }\end{array}$ & $\begin{array}{l}\text { Number of } \\
\text { Global } \\
\text { Minimum } \\
\text { Visits }\end{array}$ & $\begin{array}{c}\text { Minimum } \\
\text { Energy } \\
(\mathrm{kJ} / \mathrm{Mol})\end{array}$ & $\begin{array}{c}\text { CPU } \\
\text { Time } \\
\text { (Hours) }\end{array}$ & $\begin{array}{c}\text { Number of } \\
\text { Conformations } \\
\text { Found Per } \\
\text { Hour }\end{array}$ \\
\hline 5000 & 572 & 564 & 4 & -673.02 & 2.08 & 275 \\
\hline 5000 & 867 & 865 & 4 & -673.34 & 2.07 & 420 \\
\hline 5000 & 1107 & 1096 & 2 & -673.57 & 2.15 & 515 \\
\hline 5000 & 1314 & 1304 & 2 & -673.57 & 2.23 & 588 \\
\hline 5000 & 1480 & 1474 & 2 & -673.58 & 2.35 & 630 \\
\hline 5000 & 1628 & 1622 & 3 & -673.58 & 2.33 & 698 \\
\hline 5000 & 1765 & 1754 & 4 & -673.58 & 2.42 & 730 \\
\hline 5000 & 1899 & 1888 & 4 & -673.59 & 2.45 & 775 \\
\hline 5000 & 2013 & 2007 & 4 & -673.58 & 2.53 & 795 \\
\hline 5000 & 2097 & 2090 & 5 & -673.58 & 2.60 & 807 \\
\hline 5000 & 2154 & 2143 & 6 & -673.59 & 2.60 & 828 \\
\hline 5000 & 2244 & 2234 & 7 & -673.57 & 2.60 & 863 \\
\hline 5000 & 2318 & 2303 & 9 & -673.59 & 2.68 & 864 \\
\hline 5000 & 2387 & 2379 & 11 & -673.57 & 2.73 & 873 \\
\hline 5000 & 2466 & 2454 & 11 & -673.59 & 2.75 & 897 \\
\hline 5000 & 2513 & 2503 & 13 & -673.57 & 2.78 & 903 \\
\hline 5000 & 2551 & 2538 & 13 & -673.56 & 2.80 & 911 \\
\hline 5000 & 2623 & 2614 & 14 & -673.60 & 2.82 & 931 \\
\hline 5000 & 2682 & 2673 & 14 & -673.52 & 2.83 & 947 \\
\hline 5000 & 2733 & 2720 & 16 & -673.58 & 2.87 & 953 \\
\hline 5000 & 2774 & 2762 & 17 & -673.56 & 2.92 & 951 \\
\hline 5000 & 2818 & 2805 & 18 & -673.56 & 2.97 & 950 \\
\hline 5000 & 2843 & 2827 & 19 & -673.58 & 2.95 & 964 \\
\hline 5000 & 2895 & 2885 & 21 & -673.59 & 2.98 & 970 \\
\hline 5000 & 2934 & 2923 & 22 & -673.56 & 3.03 & 967 \\
\hline 5000 & 2968 & 2955 & 23 & -673.55 & 3.12 & 952 \\
\hline 5000 & 3003 & 2991 & 25 & -673.56 & 3.08 & 974 \\
\hline 5000 & 3011 & 3004 & 31 & -673.56 & 3.07 & 982 \\
\hline 5000 & 3037 & 3025 & 33 & -673.57 & 3.08 & 985 \\
\hline 5000 & 3063 & 3053 & 34 & -673.55 & 3.08 & 993 \\
\hline 5000 & 3072 & 3062 & 25 & -673.60 & 3.12 & 986 \\
\hline 5000 & 3120 & 3111 & 37 & -673.56 & 3.15 & 990 \\
\hline 5000 & 3130 & 3122 & 42 & -673.60 & 3.13 & 999 \\
\hline 5000 & 3152 & 3142 & 46 & -673.56 & 3.17 & 995 \\
\hline 5000 & 3173 & 3162 & 47 & -673.56 & 3.15 & 1007 \\
\hline mini & 3098 & 3093 & 47 & -673.57 & 0.95 & 3261 \\
\hline
\end{tabular}

\footnotetext{
* Within a $16.736 \mathrm{~kJ} / \mathrm{mol}$ energetic window above the lowest energy structure.
} 
S23

Table S7. LM:MC conformational search results on the OPLS2005/GBSA(water) surface for $\mathbf{6 b}$.

\begin{tabular}{|c|c|c|c|c|c|c|}
\hline $\begin{array}{l}\text { Number } \\
\text { of Steps }\end{array}$ & $\begin{array}{c}\text { Number of } \\
\text { Conformations } \\
\text { Found }\end{array}$ & $\begin{array}{c}\text { Number of } \\
\text { Conformations } \\
\text { Minimized }\end{array}$ & $\begin{array}{l}\text { Number of } \\
\text { Global } \\
\text { Minimum } \\
\text { Visits }\end{array}$ & $\begin{array}{c}\text { Minimum } \\
\text { Energy } \\
(\mathrm{kJ} / \mathrm{Mol})\end{array}$ & $\begin{array}{c}\text { CPU } \\
\text { Time } \\
\text { (Hours) }\end{array}$ & $\begin{array}{c}\text { Number of } \\
\text { Conformations } \\
\text { Found Per } \\
\text { Hour }\end{array}$ \\
\hline 5000 & $2293^{*}$ & 2272 & 1 & -651.63 & 2.22 & 1034 \\
\hline 5000 & 4663 & 4645 & 1 & -651.65 & 2.40 & 1943 \\
\hline 5000 & $2533^{\dagger}$ & 2526 & 1 & -652.29 & 2.32 & 1093 \\
\hline 5000 & 3395 & 3388 & 2 & -653.17 & 2.58 & 1314 \\
\hline 5000 & 4398 & 4395 & 3 & -653.16 & 3.03 & 1450 \\
\hline 5000 & 5300 & 5296 & 3 & -653.16 & 3.57 & 1486 \\
\hline 5000 & 6185 & 6179 & 4 & -653.16 & 4.10 & 1509 \\
\hline 5000 & 7091 & 7081 & 4 & -653.13 & 4.82 & 1472 \\
\hline 5000 & 7980 & 7972 & 6 & -653.15 & 5.58 & 1429 \\
\hline 5000 & 8781 & 8769 & 6 & -653.15 & 6.40 & 1372 \\
\hline 5000 & 9545 & 9537 & 8 & -653.15 & 7.23 & 1320 \\
\hline 5000 & $4294^{*}$ & 4290 & 9 & -653.18 & 3.72 & 1155 \\
\hline 5000 & 4619 & 4618 & 11 & -653.19 & 3.88 & 1189 \\
\hline 5000 & 4932 & 4930 & 12 & -653.16 & 4.13 & 1193 \\
\hline 5000 & 5202 & 5199 & 12 & -653.14 & 4.43 & 1173 \\
\hline 5000 & 5458 & 5455 & 15 & -653.18 & 4.67 & 1170 \\
\hline 5000 & 5699 & 5696 & 16 & -653.17 & 4.92 & 1159 \\
\hline 5000 & 5944 & 5939 & 18 & -653.17 & 5.18 & 1147 \\
\hline 5000 & 6168 & 6167 & 18 & -653.17 & 5.45 & 1132 \\
\hline 5000 & 6372 & 6369 & 21 & -653.17 & 5.68 & 1121 \\
\hline 5000 & 6567 & 6565 & 23 & -653.17 & 5.97 & 1101 \\
\hline 5000 & 6748 & 6744 & 24 & -653.17 & 6.20 & 1088 \\
\hline 5000 & 6893 & 6889 & 25 & -653.18 & 6.38 & 1080 \\
\hline 5000 & 7067 & 7063 & 26 & -653.18 & 6.60 & 1071 \\
\hline 5000 & 7206 & 7203 & 27 & -653.17 & 6.82 & 1057 \\
\hline 5000 & 7362 & 7356 & 29 & -653.16 & 7.03 & 1047 \\
\hline 5000 & 7496 & 7490 & 29 & -653.16 & 7.30 & 1027 \\
\hline 5000 & 7669 & 7669 & 30 & -653.16 & 7.43 & 1032 \\
\hline 5000 & 7819 & 7814 & 30 & -653.16 & 7.68 & 1018 \\
\hline 5000 & 7940 & 7935 & 31 & -653.16 & 7.88 & 1007 \\
\hline 5000 & 8022 & 8018 & 32 & -653.19 & 8.02 & 1001 \\
\hline 5000 & 8122 & 8119 & 32 & -653.17 & 8.17 & 995 \\
\hline 5000 & 8260 & 8256 & 33 & -653.17 & 8.38 & 985 \\
\hline 5000 & 8370 & 8367 & 35 & -653.17 & 8.58 & 975 \\
\hline 5000 & 8473 & 8467 & 35 & -653.17 & 8.72 & 972 \\
\hline $\operatorname{mini}$ & 8449 & 8447 & 35 & -653.17 & & \\
\hline mini & 8430 & 8426 & 35 & -653.17 & & \\
\hline
\end{tabular}

Within a $* 50 \mathrm{~kJ} / \mathrm{mol},{ }^{\dagger} 25 \mathrm{~kJ} / \mathrm{mol}$, or ${ }^{\star} 16.736 \mathrm{~kJ} / \mathrm{mol}$ energetic window above the lowest energy structure 
Table S8. LM:MC conformational search results on the OPLS2005/GBSA(water) surface for 4c.

\begin{tabular}{|c|c|c|c|c|c|c|}
\hline $\begin{array}{l}\text { Number } \\
\text { of Steps }\end{array}$ & $\begin{array}{c}\text { Number of } \\
\text { Conformations } \\
\text { Found }\end{array}$ & $\begin{array}{c}\text { Number of } \\
\text { Conformations } \\
\text { Minimized }\end{array}$ & $\begin{array}{l}\text { Number of } \\
\text { Global } \\
\text { Minimum } \\
\text { Visits }\end{array}$ & $\begin{array}{l}\text { Minimum } \\
\text { Energy } \\
(\mathrm{kJ} / \mathrm{Mol})\end{array}$ & $\begin{array}{c}\text { CPU } \\
\text { Time } \\
\text { (Hours) }\end{array}$ & $\begin{array}{l}\text { Number of } \\
\text { Conformations } \\
\text { Found Per } \\
\text { Hour }\end{array}$ \\
\hline 5000 & 239 & 238 & 3 & -648.86 & 3.05 & 78 \\
\hline 5000 & 526 & 525 & 9 & -648.70 & 2.98 & 176 \\
\hline 5000 & 735 & 735 & 19 & -648.87 & 3.05 & 241 \\
\hline 5000 & 885 & 885 & 22 & -648.87 & 3.12 & 284 \\
\hline 5000 & 1035 & 1034 & 29 & -648.87 & 3.13 & 330 \\
\hline 5000 & 1150 & 1150 & 33 & -648.87 & 3.18 & 361 \\
\hline 5000 & 1282 & 1281 & 39 & -648.87 & 3.27 & 392 \\
\hline 5000 & 1365 & 1365 & 42 & -648.87 & 3.30 & 414 \\
\hline 5000 & 1474 & 1474 & 45 & -648.87 & 3.30 & 447 \\
\hline 5000 & 1557 & 1557 & 47 & -648.87 & 3.37 & 462 \\
\hline 5000 & 1648 & 1648 & 50 & -648.87 & 3.42 & 482 \\
\hline 5000 & 1740 & 1740 & 55 & -648.87 & 3.43 & 507 \\
\hline 5000 & 1810 & 1810 & 61 & -648.87 & 3.47 & 522 \\
\hline 5000 & 1674 & 1674 & 1 & -649.26 & 3.45 & 485 \\
\hline 5000 & 1732 & 1731 & 1 & -649.26 & 3.42 & 507 \\
\hline 5000 & 1800 & 1800 & 1 & -649.26 & 3.48 & 517 \\
\hline 5000 & 1862 & 1862 & 1 & -649.25 & 3.50 & 532 \\
\hline 5000 & 1909 & 1906 & 1 & -649.25 & 3.50 & 545 \\
\hline 5000 & 1954 & 1953 & 1 & -649.25 & 3.52 & 556 \\
\hline 5000 & 2013 & 2012 & 3 & -649.26 & 3.55 & 567 \\
\hline 5000 & 2062 & 2061 & 5 & -649.25 & 3.58 & 575 \\
\hline 5000 & 2124 & 2124 & 7 & -649.26 & 3.60 & 590 \\
\hline 5000 & 2171 & 2170 & 9 & -649.26 & 3.60 & 603 \\
\hline 5000 & 2216 & 2215 & 12 & -649.26 & 3.65 & 607 \\
\hline 5000 & 2298 & 2298 & 12 & -649.26 & 3.68 & 624 \\
\hline 5000 & 2318 & 2318 & 15 & -649.27 & 3.75 & 618 \\
\hline 5000 & 2377 & 2377 & 17 & -649.26 & 3.72 & 640 \\
\hline 5000 & 2426 & 2423 & 19 & -649.26 & 3.78 & 641 \\
\hline 5000 & 2464 & 2464 & 20 & -649.27 & 3.80 & 648 \\
\hline 5000 & 2496 & 2494 & 20 & -649.26 & 3.85 & 648 \\
\hline 5000 & 2537 & 2537 & 21 & -649.26 & 3.85 & 659 \\
\hline 5000 & 2584 & 2580 & 22 & -649.27 & 3.90 & 663 \\
\hline 5000 & 2593 & 2591 & 23 & -649.26 & 3.88 & 668 \\
\hline 5000 & 2624 & 2622 & 25 & -649.27 & 3.92 & 670 \\
\hline 5000 & 2671 & 2670 & 27 & -649.26 & 3.92 & 682 \\
\hline mini & 2626 & 2626 & 27 & -649.27 & 0.82 & \\
\hline
\end{tabular}

\footnotetext{
* Within a $16.736 \mathrm{~kJ} / \mathrm{mol}$ energetic window above the lowest energy structure.
} 
S25

Table S9. LM:MC conformational search results on the OPLS2005/GBSA(water) surface for 5c.

\begin{tabular}{|c|c|c|c|c|c|c|}
\hline $\begin{array}{l}\text { Number } \\
\text { of Steps }\end{array}$ & $\begin{array}{c}\text { Number of } \\
\text { Conformations } \\
\text { Found }\end{array}$ & $\begin{array}{c}\text { Number of } \\
\text { Conformations } \\
\text { Minimized }\end{array}$ & $\begin{array}{l}\text { Number of } \\
\text { Global } \\
\text { Minimum } \\
\text { Visits }\end{array}$ & $\begin{array}{l}\text { Minimum } \\
\text { Energy } \\
(\mathrm{kJ} / \mathrm{Mol})\end{array}$ & $\begin{array}{c}\text { CPU } \\
\text { Time } \\
\text { (Hours) }\end{array}$ & $\begin{array}{l}\text { Number of } \\
\text { Conformations } \\
\text { Found Per } \\
\text { Hour }\end{array}$ \\
\hline 5000 & 380 & 380 & 8 & -699.68 & 2.70 & 141 \\
\hline 5000 & 748 & 747 & 14 & -699.69 & 2.77 & 270 \\
\hline 5000 & 1053 & 1048 & 19 & -699.68 & 2.88 & 365 \\
\hline 5000 & 1332 & 1331 & 21 & -699.68 & 3.02 & 442 \\
\hline 5000 & 1614 & 1611 & 23 & -699.67 & 3.08 & 523 \\
\hline 5000 & 1865 & 1864 & 26 & -699.68 & 3.27 & 571 \\
\hline 5000 & 2070 & 2067 & 29 & -699.66 & 3.35 & 618 \\
\hline 5000 & 2284 & 2284 & 34 & -699.67 & 3.45 & 662 \\
\hline 5000 & 2452 & 2451 & 38 & -699.67 & 3.60 & 681 \\
\hline 5000 & 2631 & 2629 & 40 & -699.65 & 3.70 & 711 \\
\hline 5000 & 1208 & 1206 & 1 & -703.27 & 3.58 & 337 \\
\hline 5000 & 1267 & 1266 & 1 & -703.25 & 2.98 & 425 \\
\hline 5000 & 1341 & 1340 & 1 & -703.25 & 3.02 & 445 \\
\hline 5000 & 1390 & 1389 & 1 & -703.25 & 3.05 & 456 \\
\hline 5000 & 1433 & 1433 & 1 & -703.25 & 3.08 & 465 \\
\hline 5000 & 1480 & 1477 & 1 & -703.25 & 3.12 & 475 \\
\hline 5000 & 1511 & 1509 & 1 & -703.27 & 3.13 & 482 \\
\hline 5000 & 1537 & 1536 & 1 & -703.29 & 3.15 & 488 \\
\hline 5000 & 1569 & 1569 & 1 & -703.25 & 3.10 & 506 \\
\hline 5000 & 1608 & 1605 & 1 & -703.26 & 3.18 & 505 \\
\hline 5000 & 1648 & 1647 & 1 & -703.25 & 3.17 & 520 \\
\hline 5000 & 1676 & 1675 & 1 & -703.25 & 3.20 & 524 \\
\hline 5000 & 1712 & 1707 & 1 & -703.25 & 3.22 & 532 \\
\hline 5000 & 1743 & 1743 & 1 & -703.27 & 3.22 & 542 \\
\hline 5000 & 1768 & 1766 & 1 & -703.25 & 3.27 & 541 \\
\hline 5000 & 1783 & 1783 & 1 & -703.25 & 3.23 & 551 \\
\hline 5000 & 1813 & 1813 & 1 & -703.25 & 3.27 & 555 \\
\hline 5000 & 1840 & 1835 & 1 & -703.25 & 3.30 & 558 \\
\hline 5000 & 1871 & 1870 & 1 & -703.25 & 3.33 & 561 \\
\hline 5000 & 1884 & 1883 & 3 & -703.27 & 3.30 & 571 \\
\hline 5000 & 1900 & 1900 & 4 & -703.24 & 3.35 & 567 \\
\hline 5000 & 1919 & 1919 & 4 & -703.25 & 3.32 & 579 \\
\hline 5000 & 1940 & 1937 & 4 & -703.24 & 3.33 & 582 \\
\hline 5000 & 1962 & 1961 & 5 & -703.26 & 3.38 & 580 \\
\hline 5000 & 1967 & 1967 & 6 & -703.27 & 3.35 & 587 \\
\hline mini & 1955 & 1954 & 6 & -703.24 & 0.52 & 3784 \\
\hline
\end{tabular}

\footnotetext{
* Within a $16.736 \mathrm{~kJ} / \mathrm{mol}$ energetic window above the lowest energy structure.
} 
Table S10. LM:MC conformational search results on the OPLS2005 /GBSA(water) surface for 6c.

\begin{tabular}{|c|c|c|c|c|c|c|}
\hline $\begin{array}{l}\text { Number } \\
\text { of Steps }\end{array}$ & $\begin{array}{c}\text { Number of } \\
\text { Conformations } \\
\text { Found } *\end{array}$ & $\begin{array}{c}\text { Number of } \\
\text { Conformations } \\
\text { Minimized }\end{array}$ & $\begin{array}{l}\text { Number of } \\
\text { Global } \\
\text { Minimum } \\
\text { Visits }\end{array}$ & $\begin{array}{l}\text { Minimum } \\
\text { Energy } \\
(\mathrm{kJ} / \mathrm{Mol})\end{array}$ & $\begin{array}{c}\text { CPU } \\
\text { Time } \\
\text { (Hours) }\end{array}$ & $\begin{array}{l}\text { Number of } \\
\text { Conformations } \\
\text { Found Per } \\
\text { Hour }\end{array}$ \\
\hline 5000 & $1784^{*}$ & 1775 & 1 & -667.63 & 3.00 & 595 \\
\hline 5000 & 3614 & 3603 & 1 & -667.62 & 3.38 & 1068 \\
\hline 5000 & 5371 & 5361 & 1 & -668.40 & 4.03 & 1332 \\
\hline 5000 & 6296 & 6286 & 1 & -674.20 & 4.72 & 1335 \\
\hline 5000 & 7821 & 7804 & 2 & -674.18 & 5.52 & 1418 \\
\hline 5000 & $1458^{\dagger}$ & 1457 & 3 & -674.20 & 3.25 & 449 \\
\hline 5000 & 2028 & 2027 & 3 & -674.20 & 3.25 & 624 \\
\hline 5000 & 2618 & 2617 & 3 & -674.20 & 3.48 & 752 \\
\hline 5000 & 3202 & 3202 & 3 & -674.20 & 3.73 & 858 \\
\hline 5000 & 3746 & 3745 & 3 & -674.20 & 4.02 & 933 \\
\hline 5000 & 4225 & 4223 & 3 & -674.20 & 4.38 & 964 \\
\hline 5000 & 4781 & 4778 & 3 & -674.20 & 4.75 & 1007 \\
\hline 5000 & 5345 & 5345 & 4 & -674.20 & 5.22 & 1025 \\
\hline 5000 & 4750 & 4746 & 1 & -676.15 & 5.50 & 864 \\
\hline 5000 & 5220 & 5220 & 1 & -676.14 & 5.27 & 991 \\
\hline 5000 & 5659 & 5657 & 1 & -676.14 & 5.68 & 996 \\
\hline 5000 & 6129 & 6126 & 1 & -676.14 & 6.12 & 1002 \\
\hline 5000 & 6590 & 6586 & 1 & -676.14 & 6.63 & 993 \\
\hline 5000 & 7035 & 7032 & 1 & -676.14 & 7.15 & 984 \\
\hline 5000 & 7457 & 7456 & 3 & -676.19 & 7.57 & 986 \\
\hline 5000 & 4997 & 4997 & 1 & -679.46 & 7.97 & 627 \\
\hline 5000 & 5280 & 5280 & 1 & -679.42 & 5.57 & 949 \\
\hline 5000 & 4369 & 4369 & 1 & -680.78 & 5.73 & 762 \\
\hline 5000 & 4623 & 4623 & 1 & -680.77 & 5.02 & 922 \\
\hline 5000 & 4864 & 4862 & 1 & -680.77 & 5.18 & 938 \\
\hline 5000 & 5154 & 5154 & 1 & -680.77 & 5.48 & 940 \\
\hline 5000 & $751^{*}$ & 750 & 1 & -680.79 & 2.98 & 252 \\
\hline 5000 & 820 & 818 & 1 & -680.80 & 2.90 & 283 \\
\hline 5000 & 904 & 904 & 1 & -680.81 & 2.93 & 308 \\
\hline 5000 & 963 & 963 & 1 & -680.78 & 2.92 & 330 \\
\hline 5000 & 1018 & 1018 & 1 & -680.79 & 2.92 & 349 \\
\hline 5000 & 1067 & 1067 & 1 & -680.79 & 2.95 & 362 \\
\hline 5000 & 1125 & 1124 & 1 & -680.78 & 2.97 & 379 \\
\hline 5000 & 1178 & 1178 & 1 & -680.80 & 2.92 & 404 \\
\hline 5000 & 1223 & 1223 & 1 & -680.78 & 3.02 & 405 \\
\hline 5000 & 1263 & 1263 & 1 & -680.77 & 3.02 & 419 \\
\hline 5000 & 1299 & 1299 & 1 & -680.77 & 3.05 & 426 \\
\hline
\end{tabular}




\begin{tabular}{|lllrrrr|}
5000 & 1326 & 1326 & 1 & -680.79 & 3.02 & 440 \\
5000 & 1364 & 1364 & 1 & -680.80 & 3.05 & 447 \\
5000 & 1393 & 1392 & 1 & -680.77 & 3.07 & 454 \\
mini & 1388 & 1388 & 1 & -680.77 & & \\
\hline
\end{tabular}

Within a $* 50 \mathrm{~kJ} / \mathrm{mol},{ }^{\ddagger} 25 \mathrm{~kJ} / \mathrm{mol}$, or ${ }^{\circledR} 16.736 \mathrm{~kJ} / \mathrm{mol}$ energetic window above the lowest energy structure 


\section{Cartesian coordinates for $\mathbf{4 a}$}

C

C

1.26910

0.62450

1.20900

1.39180

0.45550

1.97220

0.57000

$-0.42300$

0.39310

1.88030

0.26410

0.04110

0.65070

$-0.68810$

1.74250

2.15400

0.66730

2.14390

0.15140

0.46000

$-0.02800$

$-0.80940$

4.92090

5.23840

4.78270

4.26080

4.33980

5.03680

2. 52910

2. 47570

2. 49660

3.07600

3. 65280

3.66580

2. 97180

2.48540

4.47840

4.31100

3.74340

5.99880

5.71590

6.07340

7.30330

8.99280

9.34390

10.27610

5.83340

8.00050

7.60160

8.21620

8.25770

8.19720

7.67140

6.99590

6.86660

6.83440

6.19980

5.46620

5.75020

6.27400
$-2.45070$

$-3.63400$

$-5.00820$

$-4.78030$

$-3.65690$

$-2.34990$

$-3.30760$

$-3.77740$

$-5.71810$

$-4.53200$

$-1.94390$

$-1.57760$

$-5.83350$

$-6.79730$

$-6.03430$

$-5.31670$

$-1.14600$

$-0.27060$

$-0.98730$

$-1.15380$

$-3.36280$

$-2.54850$

$-4.23720$

$-3.07420$

$-6.25920$

$-6.11060$

$-7.33310$

$-5.63020$

$-5.25820$

$-5.13180$

$-5.90920$

$-6.93510$

$-4.00970$

$-3.11540$

$-5.56710$

$-4.69490$

$-5.25860$

$-5.95690$

$-7.15190$

$-7.53600$

$-7.65110$

$-5.72820$

$-5.93320$

$-4.64240$

$-6.33510$

$-7.77810$

$-6.70450$

$-6.56090$

$-7.49910$

$-7.99940$

$-7.90870$

$-8.05230$

$-5.76190$

$-4.65390$

$-7.55500$

$-8.31430$

$-7.73290$

$-7.71330$

$-7.58200$

$-7.40770$

$-2.57460$

$-2.02270$
1.71300

2.51540

1. 79600

0.39220

$-0.52130$

0.31320

2. 82580

3. 46220

$-0.16310$

0.56710

0.47870

$-0.28310$

2. 63680

2.17560

3. 63740

2. 76020

2. 47760

1.97240

2. 56640

3.49240

$-1.61370$

$-2.27150$

$-2.24160$

$-1.18620$

2. 15930

1.12650

2. 30030

$-1.87240$

$-2.89600$

$-1.29030$

1.70230

1.02090

$-1.31240$

$-1.93240$

2. 33940

2. 85920

$-1.30670$

$-0.75290$

$-1.83810$

$-0.83070$

$-2.47210$

3.11840

4.15250

3.04100

2.83100

2.73610

2.02700

1.50390

$-2.28070$

$-2.29650$

$-3.56580$

$-4.44060$

2.07600

1.53640

3.26110

3.96260

$-1.45160$

$-0.21830$

$-3.57200$

$-4.54980$

0.47940

1. 26240 


\section{S29}

$\begin{array}{rrrr}\mathrm{H} & 5.93660 & -3.63460 & 0.65600 \\ \mathrm{C} & 6.28410 & -2.17430 & -0.90580 \\ \mathrm{H} & 5.70080 & -2.65780 & -1.69100 \\ \mathrm{H} & 6.15890 & -1.10120 & -1.06110 \\ \mathrm{~N} & 7.69910 & -2.53480 & -1.04790 \\ \mathrm{C} & 9.91680 & -2.51350 & -0.88290 \\ \mathrm{C} & 9.57500 & -3.62800 & -1.53050 \\ \mathrm{H} & 10.24930 & -4.38120 & -1.90640 \\ \mathrm{C} & 3.62680 & -2.51910 & 1.72050 \\ \mathrm{O} & 4.20580 & -2.74400 & 2.78550 \\ \mathrm{~N} & 4.31570 & -2.35050 & 0.58800 \\ \mathrm{H} & 3.79870 & -2.20470 & -0.27360 \\ \mathrm{C} & 8.14070 & -3.65810 & -1.64210 \\ \mathrm{O} & 7.44410 & -4.53100 & -2.16680 \\ \mathrm{C} & 8.71150 & -1.79570 & -0.56070 \\ \mathrm{O} & 8.60590 & -0.72200 & 0.03990 \\ \mathrm{H} & 9.58880 & -8.66340 & 2.89250 \\ \mathrm{H} & 8.99720 & -8.22980 & -1.95470 \\ \mathrm{H} & 10.91900 & -2.19850 & -0.63820\end{array}$

\section{Cartesian coordinates for $\mathbf{4 b}$.}

\begin{tabular}{|c|c|}
\hline $\mathrm{C}$ & 4.08990 \\
\hline $\mathrm{C}$ & 3.24500 \\
\hline $\mathrm{C}$ & 1.69100 \\
\hline $\mathrm{C}$ & 1.22520 \\
\hline $\mathrm{C}$ & 1.96640 \\
\hline $\mathrm{C}$ & 3.50910 \\
\hline $\mathrm{H}$ & 3.49760 \\
\hline $\mathrm{H}$ & 3.59650 \\
\hline $\mathrm{H}$ & 0.14720 \\
\hline $\mathrm{H}$ & 1.34680 \\
\hline $\mathrm{H}$ & 4.02970 \\
\hline $\mathrm{H}$ & 3.78580 \\
\hline C & 1.10850 \\
\hline $\mathrm{H}$ & 1.44060 \\
\hline H & 1.40220 \\
\hline $\mathrm{H}$ & 0.01740 \\
\hline $\mathrm{C}$ & 5.51240 \\
\hline H & 5.50700 \\
\hline $\mathrm{H}$ & 6.17760 \\
\hline $\mathrm{H}$ & 5.97220 \\
\hline $\mathrm{C}$ & 1.64420 \\
\hline $\mathrm{H}$ & 1.95690 \\
\hline $\mathrm{H}$ & 0.57800 \\
\hline $\mathrm{H}$ & 2.15020 \\
\hline C & -0.44490 \\
\hline $\mathrm{H}$ & 0.31100 \\
\hline H & 1.32650 \\
\hline $\mathrm{H}$ & 1.05540 \\
\hline $\mathrm{C}$ & 1.16190 \\
\hline & 1.62810 \\
\hline C & 1.39760 \\
\hline & 0.17550 \\
\hline & 0.18820 \\
\hline $\mathrm{H}$ & -0.15430 \\
\hline & 2.22750 \\
\hline & 3.22910 \\
\hline & 4.23570 \\
\hline & 5.31110 \\
\hline & 4.31350 \\
\hline & 4.82950 \\
\hline & 3.98570 \\
\hline & 3.53320 \\
\hline & 1.8010 \\
\hline
\end{tabular}

$\begin{array}{ll}-4.29190 & -2.58310 \\ -3.59010 & -3.69210 \\ -3.62860 & -3.57540 \\ -5.08110 & -3.24420 \\ -5.85720 & -2.11360 \\ -5.71070 & -2.30070 \\ -4.07370 & -4.63660 \\ -2.56760 & -3.83410 \\ -5.09600 & -3.08030 \\ -5.66830 & -4.15530 \\ -6.18220 & -1.46640 \\ -6.33460 & -3.15150 \\ -3.28840 & -4.96920 \\ -3.98940 & -5.73550 \\ -2.29500 & -5.30510 \\ -3.31630 & -4.96250 \\ -4.47610 & -3.16630 \\ -5.06460 & -4.08430 \\ -4.98640 & -2.47130 \\ -3.51570 & -3.40550 \\ -7.36140 & -2.28980 \\ -7.73710 & -3.26450 \\ -7.56530 & -2.20300 \\ -7.96800 & -1.53660 \\ -1.75550 & -0.93000 \\ -1.16430 & -0.41180 \\ -5.96510 & 2.07870 \\ -4.27050 & 1.71230 \\ -2.49960 & -2.65240 \\ -1.36440 & -2.76910 \\ -5.50420 & -0.71420 \\ -5.44440 & -0.56350 \\ -2.75680 & -1.77780 \\ -3.70680 & -1.68040 \\ -5.31060 & 0.31190 \\ -5.33410 & 0.15230 \\ -1.34360 & -0.06230 \\ -1.20140 & 0.05390 \\ -3.49720 & -1.26980 \\ -4.06760 & -0.30640 \\ -2.20560 & -1.20970 \\ -1.77010 & -2.00640 \\ -5.06590 & 1.68310\end{array}$


S30

\begin{tabular}{|c|c|c|c|}
\hline C & 3.00130 & -4.67770 & 2.54970 \\
\hline $\mathrm{H}$ & 3.41040 & -3.72280 & 2.21710 \\
\hline $\mathrm{H}$ & 2.68730 & -4.55430 & 3.58740 \\
\hline O & 3.98430 & -5.69690 & 2.44700 \\
\hline $\mathrm{C}$ & 5.14340 & -5.41400 & 3.21350 \\
\hline $\mathrm{H}$ & 4.88580 & -5.37170 & 4.27310 \\
\hline $\mathrm{H}$ & 5.55850 & -4.44160 & 2.94160 \\
\hline $\mathrm{C}$ & 6.17310 & -6.52550 & 2.97040 \\
\hline $\mathrm{H}$ & 7.03640 & -6.37140 & 3.62020 \\
\hline $\mathrm{H}$ & 5.75490 & -7.49050 & 3.26140 \\
\hline $\mathrm{N}$ & 6.61220 & -6.54960 & 1.57010 \\
\hline $\mathrm{C}$ & 6.81180 & -7.05630 & -0.58410 \\
\hline C & 7.67690 & -6.07550 & -0.32200 \\
\hline $\mathrm{H}$ & 8.35110 & -5.61290 & -1.02540 \\
\hline $\mathrm{C}$ & 6.10890 & -7.37100 & 0.63180 \\
\hline O & 5.23310 & -8.22540 & 0.79600 \\
\hline $\mathrm{C}$ & 7.55330 & -5.73060 & 1.06940 \\
\hline O & 8.18090 & -4.87190 & 1.69690 \\
\hline C & 3.54240 & 0.00740 & -0.25400 \\
\hline $\mathrm{H}$ & 3.78190 & 0.67060 & 0.57880 \\
\hline $\mathrm{H}$ & 2.45980 & -0.12520 & -0.26440 \\
\hline O & 3.98080 & 0.57310 & -1.48020 \\
\hline $\mathrm{C}$ & 3.37760 & 1.82850 & -1.74650 \\
\hline $\mathrm{H}$ & 3.66760 & 2.54910 & -0.98000 \\
\hline $\mathrm{H}$ & 2.28920 & 1.74760 & -1.72040 \\
\hline $\mathrm{C}$ & 3.84790 & 2.31400 & -3.12420 \\
\hline $\mathrm{H}$ & 3.46410 & 3.31920 & -3.30730 \\
\hline $\mathrm{H}$ & 4.93510 & 2.40830 & -3.13450 \\
\hline $\mathrm{N}$ & 3.39350 & 1.41440 & -4.19110 \\
\hline C & 3.31470 & -0.19360 & -5.72270 \\
\hline C & 2.12860 & 0.41650 & -5.72170 \\
\hline $\mathrm{H}$ & 1.28530 & 0.17540 & -6.34950 \\
\hline C & -1.36040 & -2.43100 & 0.09380 \\
\hline $\mathrm{H}$ & -1.87820 & -1.67500 & 0.68640 \\
\hline $\mathrm{H}$ & -0.76990 & -3.03690 & 0.78220 \\
\hline O & -2.29550 & -3.24370 & -0.59940 \\
\hline C & -3.18220 & -3.92060 & 0.27620 \\
\hline $\mathrm{H}$ & -2.62690 & -4.50470 & 1.01260 \\
\hline $\mathrm{H}$ & -3.78580 & -3.19600 & 0.82520 \\
\hline C & -4.09170 & -4.83220 & -0.55850 \\
\hline $\mathrm{H}$ & -4.65490 & -4.23710 & -1.27930 \\
\hline $\mathrm{H}$ & -4.83920 & -5.29360 & 0.08930 \\
\hline $\mathrm{N}$ & -3.31960 & -5.87910 & -1.23820 \\
\hline C & -2.18670 & -7.02880 & -2.76540 \\
\hline C & -2.19820 & -7.75390 & -1.64590 \\
\hline $\mathrm{H}$ & -1.74260 & -8.72150 & -1.50630 \\
\hline C & 4.14450 & 0.43810 & -4.73080 \\
\hline O & 5.31080 & 0.16000 & -4.43640 \\
\hline C & 2.16170 & 1.45630 & -4.72780 \\
\hline O & 1.26060 & 2.24400 & -4.42350 \\
\hline C & -2.90480 & -5.80680 & -2.51540 \\
\hline O & -3.11550 & -4.88190 & -3.30550 \\
\hline $\mathrm{C}$ & -2.92220 & -7.01750 & -0.64400 \\
\hline O & -3.15350 & -7.34970 & 0.52270 \\
\hline $\mathrm{H}$ & -1.01920 & -1.06770 & -1.55230 \\
\hline $\mathrm{H}$ & 3.87380 & -1.81460 & 0.85230 \\
\hline $\mathrm{H}$ & 6.65770 & -7.53350 & -1.53910 \\
\hline $\mathrm{H}$ & 3.60760 & -1.01920 & -6.35200 \\
\hline $\mathrm{H}$ & -1.72010 & -7.30250 & -3.69860 \\
\hline
\end{tabular}




\section{S31}

\section{Cartesian coordinates for $\mathbf{4 c}$.}

\begin{tabular}{|c|c|c|c|}
\hline $\mathrm{C}$ & 2.35710 & -1.41460 & -10.16380 \\
\hline $\mathrm{C}$ & 3.60620 & -2.28880 & -9.83080 \\
\hline $\mathrm{C}$ & 3.82410 & -2.74160 & -8.35230 \\
\hline $\mathrm{C}$ & 3.63530 & -1.52080 & -7.40170 \\
\hline $\mathrm{C}$ & 2.36710 & -0.63430 & -7.59520 \\
\hline $\mathrm{C}$ & 2.21930 & -0.27250 & -9.10630 \\
\hline $\mathrm{H}$ & 4.48110 & -1.70130 & -10.11180 \\
\hline $\mathrm{H}$ & 3.66110 & -3.15090 & -10.49760 \\
\hline $\mathrm{H}$ & 3.72500 & -1.83310 & -6.36010 \\
\hline $\mathrm{H}$ & 4.49970 & -0.87100 & -7.54450 \\
\hline $\mathrm{H}$ & 3.00080 & 0.45560 & -9.32670 \\
\hline $\mathrm{H}$ & 1.30230 & 0.29140 & -9.27840 \\
\hline $\mathrm{C}$ & 5.30180 & -3.19040 & -8.23080 \\
\hline $\mathrm{H}$ & 5.55840 & -3.47810 & -7.20980 \\
\hline $\mathrm{H}$ & 5.51310 & -4.04770 & -8.87220 \\
\hline $\mathrm{H}$ & 5.99530 & -2.39900 & -8.51700 \\
\hline $\mathrm{C}$ & 2.62310 & -0.73440 & -11.52980 \\
\hline $\mathrm{H}$ & 3.50970 & -0.10000 & -11.50680 \\
\hline $\mathrm{H}$ & 2.78010 & -1.46540 & -12.32500 \\
\hline $\mathrm{H}$ & 1.78610 & -0.10210 & -11.83140 \\
\hline $\mathrm{C}$ & 2.59950 & 0.68910 & -6.82720 \\
\hline $\mathrm{H}$ & 2.72380 & 0.51450 & -5.75730 \\
\hline $\mathrm{H}$ & 3.49350 & 1.21010 & -7.17100 \\
\hline $\mathrm{H}$ & 1.76380 & 1.37970 & -6.94820 \\
\hline $\mathrm{C}$ & 1.52310 & -5.87520 & -8.51060 \\
\hline $\mathrm{H}$ & 1.23380 & -6.30900 & -9.46820 \\
\hline $\mathrm{H}$ & -0.76110 & -3.04350 & -6.05090 \\
\hline $\mathrm{H}$ & 0.30190 & -3.55810 & -4.76280 \\
\hline $\mathrm{C}$ & 3.00290 & -3.97520 & -7.88810 \\
\hline O & 3.06500 & -4.33100 & -6.70960 \\
\hline $\mathrm{C}$ & 1.05710 & -1.19680 & -6.98930 \\
\hline 0 & -0.02230 & -0.73170 & -7.35350 \\
\hline $\mathrm{N}$ & 2.27400 & -4.65450 & -8.77850 \\
\hline $\mathrm{H}$ & 2.22280 & -4.29460 & -9.72680 \\
\hline $\mathrm{N}$ & 1.12290 & -2.13390 & -6.04040 \\
\hline $\mathrm{H}$ & 2.03390 & -2.49410 & -5.78670 \\
\hline $\mathrm{C}$ & -1.37730 & -2.48330 & -10.18220 \\
\hline $\mathrm{H}$ & -2.04750 & -2.21980 & -9.36670 \\
\hline $\mathrm{C}$ & 1.08860 & -2.27340 & -10.41530 \\
\hline O & 1.17500 & -3.29480 & -11.10010 \\
\hline $\mathrm{N}$ & -0.08100 & -1.87290 & -9.91690 \\
\hline $\mathrm{H}$ & -0.08090 & -1.08080 & -9.28330 \\
\hline $\mathrm{C}$ & -0.02570 & -2.68670 & -5.33020 \\
\hline $\mathrm{C}$ & -0.66240 & -1.65500 & -4.38600 \\
\hline $\mathrm{H}$ & -1.07620 & -0.82320 & -4.95600 \\
\hline $\mathrm{H}$ & -1.49320 & -2.10890 & -3.84400 \\
\hline 0 & 0.32590 & -1.21460 & -3.46440 \\
\hline $\mathrm{C}$ & -0.08890 & -0.11500 & -2.66390 \\
\hline $\mathrm{H}$ & -1.14010 & -0.17860 & -2.37690 \\
\hline $\mathrm{H}$ & 0.48290 & -0.17840 & -1.73760 \\
\hline $\mathrm{C}$ & 0.24440 & 1.22730 & -3.33630 \\
\hline $\mathrm{H}$ & 1.23550 & 1.19000 & -3.79120 \\
\hline $\mathrm{C}$ & -1.96190 & -1.98150 & -11.50930 \\
\hline $\mathrm{H}$ & -2.97980 & -2.35780 & -11.62490 \\
\hline $\mathrm{H}$ & -1.37650 & -2.36320 & -12.34750 \\
\hline 0 & -1.95390 & -0.55900 & -11.51730 \\
\hline $\mathrm{C}$ & -2.56990 & -0.01830 & -12.67800 \\
\hline $\mathrm{H}$ & -2.11020 & -0.44740 & -13.57000 \\
\hline $\mathrm{H}$ & -3.63120 & -0.27020 & -12.70470 \\
\hline $\mathrm{C}$ & -2.37560 & 1.50260 & -12.70080 \\
\hline $\mathrm{H}$ & -2.68930 & 1.88960 & -13.67170 \\
\hline $\mathrm{C}$ & 0.26910 & -5.62680 & -7.65670 \\
\hline $\mathrm{H}$ & 0.55190 & -5.23220 & -6.68050 \\
\hline 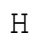 & -0.38370 & -4.89520 & -8.13470 \\
\hline
\end{tabular}


S32

$\begin{array}{ll}-0.40300 & -6.86840\end{array}$

$-6.87090$

$-7.50280$

$-6.51160$

$-5.55560$

$-6.33900$

$-7.91820$

$-6.96650$

$-6.14660$

$-8.02030$

$-8.02310$

$-6.60420$

$-3.57220$

1. 57980

2.85590

2.86140

3.60420

3.24060

3.18430

$-4.75110$

$-4.05960$

$-4.16830$

$-4.48810$

$-2.57910$

$-2.50000$

$-2.12780$

$-1.82870$

$-0.75580$

$-0.76220$

$-0.32370$

2.10190

3.51690

$-10.18160$

$-4.29720$

$-4.86930$

$-5.40430$

$-4.07840$

$-5.79700$

$-5.25220$

$-6.71400$

$-7.01790$

$-8.07930$

$-6.45510$

$-6.64710$

$-5.60450$

$-7.22550$

$-6.86480$

$-7.84260$

$-6.53040$

$-6.03340$

$-7.64340$

$-3.04230$

$-3.91240$

$-3.84970$

4.08140

5.17100

3.76360

3.67060

3.50790

2.58600

1.88950

2.41870

1. 65090

0.93850

0.14740

$-11.67750$

$-11.66270$

$-10.52930$

$-10.52690$

$-9.56470$

$-10.67090$

$-11.38730$

$-10.43880$

$-10.08360$

$-7.01210$

$-9.05890$

$-8.55090$

$-9.05490$

$-8.07470$

$-9.15310$

$-5.88270$

$-4.67800$

$-7.21630$

$-6.42010$

$-8.07040$

$-8.16290$

$-0.00730$

$-6.17120$

$-5.92200$

$-5.88510$

$-5.34550$

0.27610

$-1.31990$

$-3.54460$

$-1.55520$

$-7.17550$

$-8.31880$

$-0.87950$

$-3.35020$

$-2.00190$

1.00500

2.61080

3.45940

4.21890

$-11.54960$

5.14270

$-12.33030$

$-9.96390$

$-9.08410$

2.00370

$-2.56480$

1.99860

$-12.57830$

$-6.39760$

$-6.08660$

$-8.60290$

$-11.94100$

$-10.04250$

$-12.62370$

$-11.50520$ 


\section{S33}

\section{Cartesian coordinates for $\mathbf{5 a}$.}

\begin{tabular}{|c|c|c|c|}
\hline $\mathrm{C}$ & 2.41860 & 1.04740 & 0.05190 \\
\hline $\mathrm{C}$ & 1.28810 & 2.08010 & -0.14790 \\
\hline $\mathrm{C}$ & -0.04670 & 1.40010 & -0.52280 \\
\hline $\mathrm{C}$ & 0.13540 & 0.53010 & -1.78550 \\
\hline $\mathrm{C}$ & 1.25530 & -0.51690 & -1.60090 \\
\hline $\mathrm{C}$ & 2.57800 & 0.18070 & -1.21610 \\
\hline $\mathrm{H}$ & 2.13760 & 0.39170 & 0.87810 \\
\hline $\mathrm{H}$ & 1.57150 & 2.79140 & -0.92620 \\
\hline $\mathrm{H}$ & 1.15910 & 2.66510 & 0.76460 \\
\hline $\mathrm{H}$ & -0.33760 & 0.74600 & 0.30120 \\
\hline $\mathrm{H}$ & -0.80330 & 0.02650 & -2.02340 \\
\hline $\mathrm{H}$ & 0.36310 & 1.16710 & -2.64260 \\
\hline $\mathrm{H}$ & 0.96940 & -1.17880 & -0.78140 \\
\hline $\mathrm{H}$ & 2.92320 & 0.80050 & -2.04600 \\
\hline $\mathrm{H}$ & 3.35510 & -0.56850 & -1.05410 \\
\hline $\mathrm{C}$ & 3.73980 & 1.74230 & 0.41470 \\
\hline 0 & 4.26720 & 2.53170 & -0.37120 \\
\hline $\mathrm{N}$ & 4.27190 & 1.44890 & 1.60670 \\
\hline $\mathrm{H}$ & 3.77810 & 0.78280 & 2.18740 \\
\hline $\mathrm{C}$ & 5.51370 & 1.99730 & 2.13820 \\
\hline $\mathrm{H}$ & 5.90540 & 1.29110 & 2.87180 \\
\hline $\mathrm{H}$ & 6.26350 & 2.06750 & 1.34740 \\
\hline $\mathrm{C}$ & 5.28210 & 3.37150 & 2.79470 \\
\hline $\mathrm{H}$ & 4.90250 & 4.08140 & 2.05720 \\
\hline $\mathrm{H}$ & 4.50360 & 3.29560 & 3.55620 \\
\hline $\mathrm{N}$ & 6.51350 & 3.89360 & 3.39690 \\
\hline $\mathrm{C}$ & 8.45590 & 4.93580 & 3.68790 \\
\hline $\mathrm{C}$ & 8.14820 & 4.33470 & 4.83820 \\
\hline $\mathrm{H}$ & 8.73260 & 4.35230 & 5.74470 \\
\hline $\mathrm{C}$ & 7.40730 & 4.65640 & 2.74230 \\
\hline O & 7.32810 & 5.02680 & 1.56680 \\
\hline $\mathrm{C}$ & 6.89320 & 3.65190 & 4.66450 \\
\hline O & 6.27750 & 2.97350 & 5.49300 \\
\hline $\mathrm{H}$ & 9.33510 & 5.52920 & 3.49230 \\
\hline $\mathrm{C}$ & -1.15750 & 2.44120 & -0.72820 \\
\hline O & -1.05300 & 3.30220 & -1.60400 \\
\hline $\mathrm{N}$ & -2.22140 & 2.36010 & 0.07890 \\
\hline $\mathrm{H}$ & -2.22280 & 1.62780 & 0.77770 \\
\hline $\mathrm{C}$ & -3.37760 & 3.24750 & 0.04810 \\
\hline $\mathrm{H}$ & -3.83910 & 3.23170 & 1.03660 \\
\hline $\mathrm{H}$ & -3.05790 & 4.27710 & -0.12610 \\
\hline $\mathrm{C}$ & -4.39490 & 2.80350 & -1.02020 \\
\hline $\mathrm{H}$ & -3.93660 & 2.83190 & -2.01070 \\
\hline $\mathrm{H}$ & -4.67690 & 1.76150 & -0.85760 \\
\hline $\mathrm{N}$ & -5.59390 & 3.64840 & -1.00440 \\
\hline $\mathrm{C}$ & -7.03720 & 5.29010 & -1.41090 \\
\hline $\mathrm{C}$ & -7.60550 & 4.46730 & -0.52820 \\
\hline $\mathrm{H}$ & -8.58620 & 4.57620 & -0.09260 \\
\hline $\mathrm{C}$ & -5.73110 & 4.77460 & -1.72710 \\
\hline 0 & -4.89250 & 5.25440 & -2.49640 \\
\hline $\mathrm{C}$ & -6.68070 & 3.39960 & -0.25190 \\
\hline 0 & -6.83130 & 2.44560 & 0.51800 \\
\hline $\mathrm{H}$ & -7.47350 & 6.18730 & -1.82100 \\
\hline $\mathrm{C}$ & 1.43060 & -1.36640 & -2.86890 \\
\hline 0 & 1.74660 & -0.83780 & -3.93660 \\
\hline $\mathrm{N}$ & 1.22680 & -2.68320 & -2.74960 \\
\hline $\mathrm{H}$ & 0.96090 & -3.03400 & -1.83800 \\
\hline $\mathrm{C}$ & 1.34130 & -3.65920 & -3.82650 \\
\hline $\mathrm{H}$ & 0.92790 & -3.25050 & -4.75090 \\
\hline $\mathrm{H}$ & 0.72280 & -4.51950 & -3.56670 \\
\hline $\mathrm{C}$ & 2.80380 & -4.09840 & -4.02970 \\
\hline $\mathrm{H}$ & 3.42000 & -3.23940 & -4.30230 \\
\hline $\mathrm{H}$ & 3.21700 & -4.46870 & -3.08950 \\
\hline - & 2.91510 & -5.13540 & -5.06120 \\
\hline
\end{tabular}


S34

$\begin{array}{llll}\mathrm{C} & 3.13670 & -6.16300 & -7.02040 \\ \mathrm{C} & 2.98320 & -7.10580 & -6.08930 \\ \mathrm{H} & 2.97100 & -8.17150 & -6.25490 \\ \mathrm{C} & 3.09140 & -4.87980 & -6.36990 \\ \mathrm{O} & 3.18750 & -3.76310 & -6.88870 \\ \mathrm{C} & 2.83490 & -6.45510 & -4.81400 \\ \mathrm{O} & 2.66250 & -6.98070 & -3.70960 \\ \mathrm{H} & 3.27150 & -6.32570 & -8.07810\end{array}$

\section{Cartesian coordinates for $\mathbf{5 b}$.}

\begin{tabular}{|c|c|c|c|}
\hline $\mathrm{C}$ & -2.99100 & -3.03240 & 1.81640 \\
\hline $\mathrm{C}$ & -1.91030 & -2.45160 & 0.87280 \\
\hline $\mathrm{C}$ & -1.56210 & -0.98840 & 1.2105 \\
\hline $\mathrm{C}$ & -2.83490 & -0.12250 & 1.1899 \\
\hline $\mathrm{C}$ & -3.90630 & -0.64960 & 2.1602 \\
\hline $\mathrm{C}$ & -4.24300 & -2.12070 & 1.8295 \\
\hline $\mathrm{H}$ & -2.58750 & -3.06800 & 2.8297 \\
\hline $\mathrm{H}$ & -2.25150 & -2.52390 & -0.1595 \\
\hline $\mathrm{H}$ & -1.00100 & -3.05220 & 0.9272 \\
\hline $\mathrm{H}$ & -1.14430 & -0.95600 & 2.2184 \\
\hline $\mathrm{H}$ & -2.57650 & 0.90490 & 1.4489 \\
\hline $\mathrm{H}$ & -3.24720 & -0.09290 & 0.1801 \\
\hline $\mathrm{H}$ & -3.50890 & -0.61360 & 3.1762 \\
\hline $\mathrm{H}$ & -4.73470 & -2.17110 & 0.8567 \\
\hline $\mathrm{H}$ & -4.96660 & -2.49930 & 2.5535 \\
\hline $\mathrm{C}$ & -3.33750 & -4.47730 & 1.4117 \\
\hline O & -4.47770 & -4.78040 & 1.0560 \\
\hline N & -2.33990 & -5.36690 & 1.4573 \\
\hline $\mathrm{H}$ & -1.44170 & -5.04530 & 1.7920 \\
\hline $\mathrm{C}$ & -2.39450 & -6.72430 & 0.9219 \\
\hline $\mathrm{H}$ & -1.77610 & -7.36530 & 1.5512 \\
\hline $\mathrm{H}$ & -3.40520 & -7.13350 & 0.9691 \\
\hline $\mathrm{H}$ & -0.84560 & -6.37290 & -0.5528 \\
\hline $\mathrm{H}$ & -1.83180 & -7.79050 & -0.8757 \\
\hline $\mathrm{C}$ & -0.52050 & -0.43160 & 0.2304 \\
\hline O & -0.83100 & -0.20840 & -0.9399 \\
\hline $\mathrm{N}$ & 0.70180 & -0.19530 & 0.7153 \\
\hline $\mathrm{H}$ & 0.88100 & -0.43480 & 1.6810 \\
\hline $\mathrm{C}$ & 1.80570 & 0.40330 & -0.0289 \\
\hline $\mathrm{H}$ & 1.70690 & 0.23410 & -1.1021 \\
\hline $\mathrm{H}$ & 2.72380 & -0.10620 & 0.2655 \\
\hline $\mathrm{C}$ & 1.93860 & 1.90320 & 0.2915 \\
\hline $\mathrm{H}$ & 2.09540 & 2.02290 & 1.3638 \\
\hline C & -5.14740 & 0.25470 & 2.1035 \\
\hline O & -6.07200 & 0.01590 & 1.3247 \\
\hline $\mathrm{N}$ & -5.15360 & 1.31020 & 2.9195 \\
\hline $\mathrm{H}$ & -4.32960 & 1.47370 & 3.4950 \\
\hline $\mathrm{C}$ & -6.20810 & 2.31320 & 2.9844 \\
\hline $\mathrm{H}$ & -6.52860 & 2.59680 & 1.9805 \\
\hline $\mathrm{C}$ & -1.86610 & -6.75910 & -0.5211 \\
\hline O & -2.71530 & -5.98990 & -1.3643 \\
\hline $\mathrm{C}$ & -2.22690 & -5.89380 & -2.6931 \\
\hline $\mathrm{H}$ & -1.26560 & -5.37740 & -2.7053 \\
\hline $\mathrm{H}$ & -2.06100 & -6.89090 & -3.1046 \\
\hline $\mathrm{C}$ & -3.25700 & -5.15150 & -3.5590 \\
\hline $\mathrm{H}$ & -2.89370 & -5.09370 & -4.5865 \\
\hline $\mathrm{H}$ & -4.17870 & -5.73370 & -3.6080 \\
\hline $\mathrm{N}$ & -3.52300 & -3.79430 & -3.0632 \\
\hline C & -4.48920 & -2.03090 & -2.1150 \\
\hline C & -3.32340 & -1.62550 & -2.6207 \\
\hline $\mathrm{H}$ & -2.91750 & -0.62730 & -2.5754 \\
\hline O & 0.77890 & 2.65250 & -0.0658 \\
\hline $\mathrm{C}$ & 0.66130 & 2.89700 & -1.4623 \\
\hline $\mathrm{H}$ & 0.47330 & 1.97200 & -2.0079 \\
\hline
\end{tabular}


S35

$$
\begin{array}{r}
1.59380 \\
-0.46530 \\
-0.24480
\end{array}
$$

3.31140

3.91070

4.84160

4.17300

3.38820

2.31160

3. 00010

3.03180

3.54840

4.28620

3.27200

4.10100

5.21230

5.82170

5.85460

4.75370

5.62390

4.10470

4.08330

3.71160

2.53610

1.58100

$-2.75840$

$-2.79420$

$-3.43460$

$-4.16200$

2.55430

2.11220

3.70030

4.44630

2.76150

1.92410

4.72580

5.93910

2.31900

1.89030

$-1.41950$

1.68300

3.88350

$-1.84910$

$-1.71020$

$-1.18500$

$-2.76940$

$-1.31570$

$-1.33340$

$-0.19310$

0.58010

3. 74010

3.80990

4. 75890

3.03820

3.69600

2.94540

4.14190

4.75720

5.31950

5.49830

4.14320

3.17130

3.28360

2.95930

$-3.23570$

$-3.81560$

$-2.40120$

$-2.13230$

$-2.07330$

$-3.18300$

$-0.16540$

0.71820

3.91060

4.18450

3.72070

3.80400

$-0.18610$

3.48980

$-1.58520$

$-1.65230$

2.73690

\section{Cartesian coordinates for $\mathbf{5 c}$.}

$\begin{array}{ll}\mathrm{C} & -5.82860 \\ \mathrm{C} & -4.80430 \\ \mathrm{C} & -3.47390 \\ \mathrm{C} & -3.70510 \\ \mathrm{C} & -4.73160 \\ \mathrm{C} & -6.06520 \\ \mathrm{H} & -5.39270 \\ \mathrm{H} & -5.19500 \\ \mathrm{H} & -4.64140 \\ \mathrm{H} & -3.14630 \\ \mathrm{H} & -2.76340 \\ \mathrm{H} & -4.05920 \\ \mathrm{H} & -4.32590 \\ \mathrm{H} & -6.49470 \\ \mathrm{H} & -6.79240 \\ \mathrm{C} & -7.12700 \\ \mathrm{O} & -7.89540 \\ \mathrm{~N} & -7.36420 \\ \mathrm{H} & -6.67160 \\ \mathrm{C} & -8.53160 \\ \mathrm{H} & -9.41190 \\ \mathrm{H} & -8.35000\end{array}$

$-6.37210$

$-2.68420$

$-7.37800$

$-2.11720$

$-2.88990$

$-7.25980$

$-4.39140$

$-6.52730$

$-4.95310$

$-6.64350$

$-4.18470$

$-2.61450$

-5.37810
-8.39440

$-2.19130$

$-7.18940$

$-1.05510$

$-2.78600$

$-4.93650$

$-4.53720$

$-4.76770$

$-4.32560$

$-4.58570$

$-1.87000$

$-1.88730$

$-1.15900$

$-1.17470$

$-0.31910$

$-0.72180$

$-5.51030$

0.67160 
S36

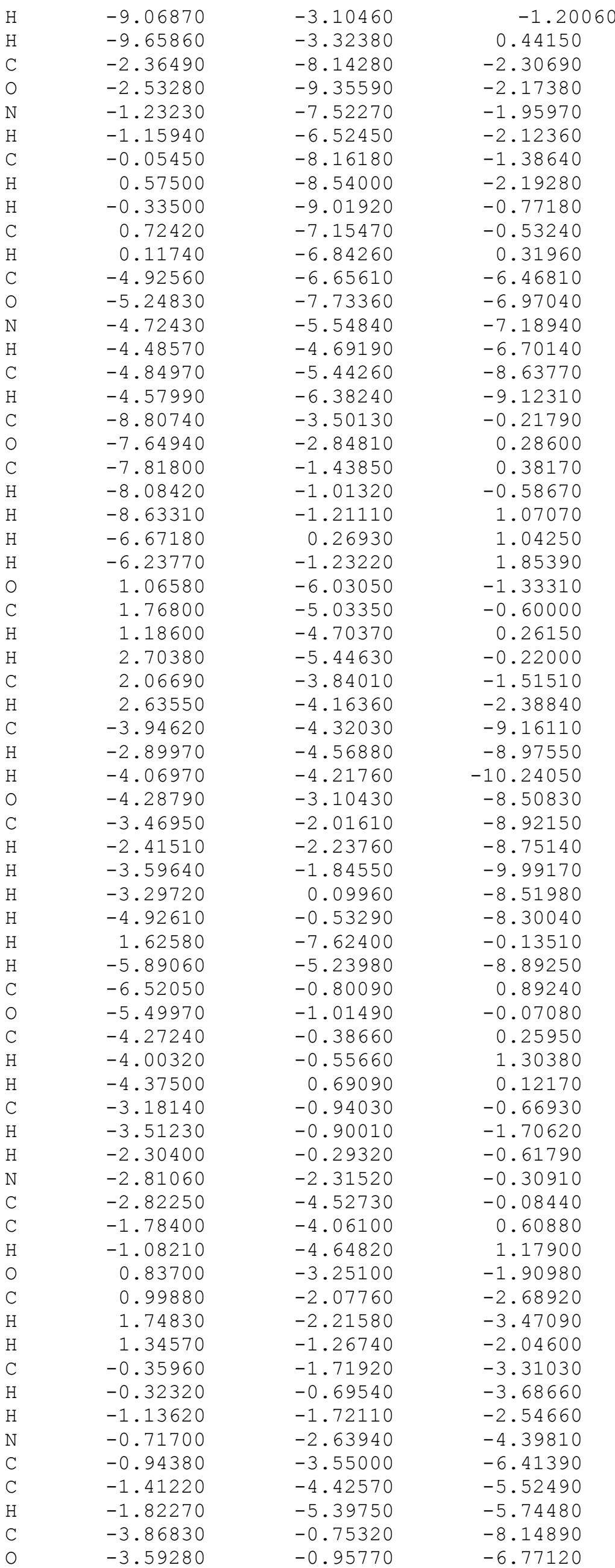


S37

$\begin{array}{ll}\mathrm{C} & -3.84170 \\ \mathrm{H} & -4.80140 \\ \mathrm{H} & -3.06710 \\ \mathrm{C} & -3.81610 \\ \mathrm{H} & -3.73830 \\ \mathrm{H} & -2.92040 \\ \mathrm{~N} & -5.01550 \\ \mathrm{C} & -7.05080 \\ \mathrm{C} & -6.49510 \\ \mathrm{H} & -6.95140 \\ \mathrm{C} & -1.76320 \\ \mathrm{O} & -0.96650 \\ \mathrm{C} & -5.17440 \\ \mathrm{O} & -4.34960 \\ \mathrm{C} & -6.10430 \\ \mathrm{O} & -6.24970 \\ \mathrm{C} & -0.48890 \\ \mathrm{O} & 0.02380 \\ \mathrm{C} & -1.27420 \\ \mathrm{O} & -1.59100 \\ \mathrm{C} & -3.49700 \\ \mathrm{O} & -4.50160 \\ \mathrm{H} & 2.67410 \\ \mathrm{H} & -3.11150 \\ \mathrm{H} & -0.90380 \\ \mathrm{H} & -8.03890\end{array}$

0.18590

0.64530

0.93020

$-0.24230$

0.64560

$-0.82600$

$-1.00890$

$-1.54440$

$-2.65290$

$-3.62830$

$-2.62860$

$-1.83270$

$-2.32270$

$-3.07680$

$-0.46990$

0.71320

$-2.38710$

$-1.36560$

$-3.84920$

$-4.35120$

$-3.40790$

$-3.42990$

$-3.10930$

$-5.56120$

$-3.68540$

$-1.45920$
$-5.97090$

$-6.21550$

$-6.16190$

$-4.49590$

$-3.86600$

$-4.28780$

$-4.13390$

$-3.41630$

$-3.90510$

$-3.94650$

0.47360

0.97820

$-4.37230$

$-4.89180$

$-3.55800$

$-3.23860$

$-5.69890$

$-6.16420$

$-4.21340$

$-3.13340$

$-0.68740$

$-1.40090$

$-0.97840$

$-0.17910$

$-7.48290$

$-2.99310$

\section{Cartesian coordinates for $\mathbf{6 a}$.}

$\begin{array}{ll}\mathrm{C} & 2.22610 \\ \mathrm{C} & 3.28010 \\ \mathrm{C} & 4.61590 \\ \mathrm{C} & 4.90360 \\ \mathrm{C} & 3.83400 \\ \mathrm{C} & 2.49270 \\ \mathrm{H} & 1.20500 \\ \mathrm{H} & 5.43450 \\ \mathrm{C} & 2.97060 \\ \mathrm{O} & 1.95490 \\ \mathrm{~N} & 3.82910 \\ \mathrm{H} & 4.60340 \\ \mathrm{C} & 3.71840 \\ \mathrm{H} & 4.25400 \\ \mathrm{H} & 2.67530 \\ \mathrm{C} & 4.30660 \\ \mathrm{H} & 3.76760 \\ \mathrm{H} & 5.33940 \\ \mathrm{C} & 3.56470 \\ \mathrm{C} & 4.77270 \\ \mathrm{H} & 5.31770 \\ \mathrm{~N} & 4.25310 \\ \mathrm{C} & 5.23100 \\ \mathrm{O} & 6.29570 \\ \mathrm{C} & 3.21230 \\ \mathrm{O} & 2.17230 \\ \mathrm{C} & 6.33120 \\ \mathrm{O} & 7.19850 \\ \mathrm{~N} & 6.59380 \\ \mathrm{H} & 5.81970 \\ \mathrm{C} & 7.90350 \\ \mathrm{H} & 8.67380 \\ \mathrm{H} & 7.90330 \\ \mathrm{C} & 8.21810 \\ \mathrm{H} & 8.22230 \\ \mathrm{H} & 7.42610\end{array}$

$\begin{array}{rr}8.98840 & -1.17470 \\ 8.31260 & -0.52080 \\ 8.67130 & -0.81620 \\ 9.66380 & -1.77920 \\ 10.32780 & -2.42390 \\ 10.00530 & -2.12090 \\ 8.71200 & -0.94810 \\ 8.18440 & -0.30310 \\ 7.25740 & 0.50390 \\ 7.32500 & 1.19930 \\ 6.23270 & 0.55940 \\ 6.24890 & -0.09000 \\ 5.08040 & 1.44480 \\ 4.24950 & 0.98350 \\ 4.76760 & 1.52600 \\ 5.38550 & 2.83540 \\ 6.21430 & 3.29850 \\ 5.72570 & 2.73800 \\ 2.68820 & 5.17880 \\ 2.31570 & 4.75320 \\ 1.43860 & 5.06490 \\ 4.20990 & 3.71110 \\ 3.29040 & 3.79850 \\ 3.29720 & 3.17250 \\ 3.91290 & 4.50980 \\ 4.56870 & 4.62640 \\ 10.03200 & -2.07200 \\ 9.96050 & -1.19880 \\ 10.37600 & -3.33800 \\ 10.35390 & -3.98770 \\ 10.73770 & -3.86470 \\ 10.09830 & -3.42850 \\ 10.53230 & -4.93610 \\ 12.22370 & -3.60840 \\ 12.42810 & -2.53600 \\ 12.85040 & -4.02290\end{array}$


S38

$\begin{array}{rc}12.60770 & -4.19010 \\ 12.98380 & -4.46640 \\ 13.31340 & -5.60540 \\ 13.04930 & -4.26660 \\ 13.69460 & -6.49690 \\ 12.52120 & -3.53980 \\ 12.11870 & -2.38400 \\ 13.07190 & -5.44320 \\ 13.24280 & -6.27230 \\ 10.71510 & -2.83000 \\ 11.11650 & -3.98780 \\ 10.92670 & -2.10530 \\ 10.60100 & -1.14840 \\ 11.62080 & -2.56470 \\ 12.02200 & -1.68950 \\ 12.47940 & -3.18020 \\ 10.67210 & -3.33970 \\ 10.27400 & -4.21780 \\ 9.80390 & -2.72470 \\ 11.35060 & -3.75190 \\ 12.16240 & -3.77050 \\ 12.52750 & -4.91320 \\ 13.10820 & -5.70310 \\ 12.39320 & -3.46560 \\ 11.40160 & -3.00520 \\ 10.89600 & -1.88880 \\ 12.01160 & -4.91470 \\ 12.14200 & -5.79000 \\ 11.10750 & -3.14630 \\ 2.16790 & 5.89830 \\ & \end{array}$

$$
12.60770
$$$$
11.68280
$$$$
11.07190
$$$$
12.74070
$$$$
11.54450
$$$$
10.68310
$$$$
10.84870
$$$$
9.66230
$$$$
8.76280
$$$$
1.37360
$$$$
\text { 1. } 50800
$$$$
0.26910
$$$$
0.28060
$$$$
-0.92710
$$$$
-1.44000
$$$$
-0.65030
$$$$
-1.86100
$$$$
-1.34880
$$$$
-2.10590
$$$$
-3.09440
$$$$
-5.16440
$$$$
-4.58110
$$$$
-5.03100
$$$$
-6.17300
$$$$
-4.21200
$$$$
-4.36620
$$$$
-3.23740
$$$$
-2.37600
$$$$
4.03570
$$$$
\mathrm{H}
$$$$
\text { 2. } 95230
$$

$$
\begin{aligned}
& -4.46640 \\
& -5.60540 \\
& -4.26660 \\
& -6.49690 \\
& -3.53980 \\
& -2.38400 \\
& -5.44320 \\
& -6.27230 \\
& -2.83000 \\
& -3.98780 \\
& -2.10530 \\
& -1.14840 \\
& -2.56470 \\
& -1.68950 \\
& -3.18020 \\
& -3.33970 \\
& -4.21780 \\
& -2.72470 \\
& -3.75190 \\
& -3.77050 \\
& -4.91320 \\
& -5.70310 \\
& -3.46560 \\
& -3.00520 \\
& -1.88880 \\
& -4.91470 \\
& -5.79000 \\
& -3.14630 \\
& 5.89830
\end{aligned}
$$

\section{Cartesian coordinates for $\mathbf{6 b}$.}

$\begin{array}{rr}\mathrm{C} & 3.24160 \\ \mathrm{C} & 3.93690 \\ \mathrm{C} & 3.20270 \\ \mathrm{C} & 1.78960 \\ \mathrm{C} & 1.11360 \\ \mathrm{C} & 1.82830 \\ \mathrm{H} & 3.80480 \\ \mathrm{H} & 3.72680 \\ \mathrm{C} & 5.43920 \\ \mathrm{O} & 6.08330 \\ \mathrm{~N} & 5.99990 \\ \mathrm{H} & 5.37780 \\ \mathrm{H} & 8.03600 \\ \mathrm{H} & 7.74570 \\ \mathrm{O} & -0.09980 \\ \mathrm{C} & 1.07940 \\ \mathrm{O} & -0.02890 \\ \mathrm{~N} & 1.64480 \\ \mathrm{H} & 2.53640 \\ \mathrm{H} & 1.73600 \\ \mathrm{H} & 0.46180 \\ \mathrm{H} & 0.03170 \\ \mathrm{C} & 1.00120 \\ \mathrm{C} & 0.85740 \\ \mathrm{H} & 0.13620 \\ \mathrm{H} & 1.59700 \\ \mathrm{C} & 0.15830 \\ \mathrm{H} & -0.58340 \\ \mathrm{H} & -0.36940 \\ \mathrm{O} & 1.14370 \\ \mathrm{C} & 0.58880\end{array}$

$\begin{array}{rr}2.14760 & 1.75670 \\ 3.11230 & 2.51840 \\ 4.05940 & 3.27020 \\ 4.06940 & 3.23710 \\ 3.09440 & 2.47260 \\ 2.14170 & 1.71500 \\ 1.41380 & 1.19580 \\ 4.78430 & 3.87790 \\ 3.11450 & 2.53190 \\ 2.06840 & 2.43360 \\ 4.32490 & 2.56990 \\ 5.12360 & 2.57440 \\ 3.74690 & 2.31820 \\ 5.13790 & 3.34110 \\ 4.81390 & 4.48620 \\ 1.14250 & 0.88120 \\ 0.72540 & 1.22310 \\ 0.84220 & -0.29120 \\ 1.26970 & -0.49450 \\ -0.16400 & -2.10800 \\ -0.73230 & -1.05080 \\ 3.09290 & 2.44740 \\ 5.09060 & 4.00630 \\ 7.50900 & 4.55410 \\ 7.25100 & 5.33170 \\ 8.15600 & 5.02570 \\ 8.25590 & 3.41070 \\ 7.61010 & 2.93680 \\ 9.12810 & 3.79990 \\ 8.66470 & 2.47140 \\ 9.25800 & 1.30980\end{array}$




\begin{tabular}{|c|c|c|c|}
\hline $\mathrm{H}$ & -0.05060 & 10.10020 & 1.58010 \\
\hline $\mathrm{H}$ & -0.02730 & 8.52920 & 0.78050 \\
\hline $\mathrm{C}$ & 1.75130 & 9.72560 & 0.42280 \\
\hline $\mathrm{H}$ & 2.31610 & 10.50810 & 0.93210 \\
\hline $\mathrm{H}$ & 2.44800 & 8.89940 & 0.28280 \\
\hline $\mathrm{N}$ & 1.29040 & 10.21680 & -0.87940 \\
\hline $\mathrm{C}$ & 0.80220 & 10.35330 & -3.04380 \\
\hline $\mathrm{C}$ & 0.43080 & 11.50200 & -2.47630 \\
\hline $\mathrm{H}$ & -0.02430 & 12.34330 & -2.97500 \\
\hline $\mathrm{N}$ & 1.52780 & 6.31870 & 4.04200 \\
\hline $\mathrm{H}$ & 2.40780 & 6.46670 & 3.55660 \\
\hline $\mathrm{C}$ & 0.74210 & 11.42910 & -1.07300 \\
\hline O & 0.55230 & 12.28780 & -0.20640 \\
\hline $\mathrm{C}$ & 1.36350 & 9.51060 & -2.02140 \\
\hline O & 1.82570 & 8.37270 & -2.14490 \\
\hline $\mathrm{C}$ & 7.41730 & 4.63910 & 2.42870 \\
\hline $\mathrm{C}$ & 7.60880 & 5.57450 & 1.22810 \\
\hline $\mathrm{H}$ & 8.60720 & 6.01470 & 1.23800 \\
\hline $\mathrm{H}$ & 7.49890 & 5.01770 & 0.29590 \\
\hline 0 & 6.60680 & 6.57700 & 1.31360 \\
\hline $\mathrm{C}$ & 6.66090 & 7.55080 & 0.28650 \\
\hline $\mathrm{H}$ & 7.51450 & 8.21250 & 0.44130 \\
\hline $\mathrm{H}$ & 6.78300 & 7.07840 & -0.68980 \\
\hline $\mathrm{C}$ & 5.33680 & 8.33000 & 0.31390 \\
\hline $\mathrm{H}$ & 5.35190 & 9.10820 & -0.45130 \\
\hline $\mathrm{H}$ & 4.51760 & 7.66760 & 0.03550 \\
\hline $\mathrm{N}$ & 5.07440 & 8.91830 & 1.63380 \\
\hline $\mathrm{C}$ & 5.04600 & 10.31840 & 3.35870 \\
\hline $\mathrm{C}$ & 4.40730 & 9.20960 & 3.73390 \\
\hline $\mathrm{H}$ & 3.95660 & 9.02710 & 4.69640 \\
\hline $\mathrm{C}$ & 5.49080 & 10.14160 & 2.00150 \\
\hline 0 & 6.12430 & 10.93660 & 1.30110 \\
\hline $\mathrm{C}$ & 4.42220 & 8.28850 & 2.62860 \\
\hline 0 & 3.93990 & 7.15150 & 2.58800 \\
\hline $\mathrm{C}$ & 0.97690 & 0.16510 & -1.39790 \\
\hline $\mathrm{C}$ & -0.00600 & 1.12130 & -2.09210 \\
\hline $\mathrm{H}$ & -0.39400 & 0.67630 & -3.00960 \\
\hline $\mathrm{H}$ & -0.85940 & 1.31170 & -1.43870 \\
\hline $\mathrm{O}$ & 0.68170 & 2.33280 & -2.37900 \\
\hline $\mathrm{C}$ & -0.17700 & 3.37230 & -2.81880 \\
\hline $\mathrm{H}$ & -0.65660 & 3.09060 & -3.75760 \\
\hline $\mathrm{H}$ & -0.96970 & 3.54890 & -2.08960 \\
\hline $\mathrm{C}$ & 0.66030 & 4.64530 & -3.01180 \\
\hline $\mathrm{H}$ & 1.37990 & 4.49310 & -3.81800 \\
\hline $\mathrm{H}$ & 0.01650 & 5.46180 & -3.34370 \\
\hline $\mathrm{N}$ & 1.34660 & 5.03540 & -1.77280 \\
\hline $\mathrm{C}$ & 1.76310 & 5.86620 & 0.24480 \\
\hline $\mathrm{C}$ & 2.89710 & 5.33230 & -0.20840 \\
\hline $\mathrm{H}$ & 3.84320 & 5.31170 & 0.30980 \\
\hline $\mathrm{C}$ & 0.74620 & 5.67350 & -0.75380 \\
\hline $\mathrm{O}$ & -0.43750 & 6.01860 & -0.70480 \\
\hline $\mathrm{C}$ & 2.64410 & 4.78980 & -1.51670 \\
\hline O & 3.44460 & 4.21920 & -2.26290 \\
\hline $\mathrm{H}$ & 0.70350 & 10.09380 & -4.08610 \\
\hline $\mathrm{H}$ & 5.20680 & 11.19810 & 3.96200 \\
\hline $\mathrm{H}$ & 1.62630 & 6.35820 & 1.19370 \\
\hline
\end{tabular}

\section{Cartesian coordinates for $\mathbf{6 c}$.}

$\begin{array}{ll}\text { C } & -5.08810 \\ \text { C } & -5.19920 \\ \text { C } & -4.63490 \\ \text { C } & -3.89720 \\ \text { C } & -3.78380 \\ \text { C } & -4.36970\end{array}$

$\begin{array}{ll}4.53170 & 3.91550 \\ 5.66840 & 3.08620 \\ 5.63400 & 1.79220 \\ 4.51210 & 1.35450 \\ 3.38900 & 2.20270 \\ 3.39250 & 3.48990\end{array}$




\section{$\mathrm{S} 40$}

\begin{tabular}{|c|c|c|c|}
\hline $\mathrm{H}$ & -5.53950 & 4.54480 & 4.8985 \\
\hline $\mathrm{H}$ & -4.75860 & 6.48240 & 1.13370 \\
\hline $\mathrm{C}$ & -5.91340 & 6.89360 & 3.57770 \\
\hline 0 & -6.88710 & 6.80700 & 4.32840 \\
\hline $\mathrm{N}$ & -5.36880 & 8.05490 & 3.19910 \\
\hline $\mathrm{H}$ & -4.52790 & 8.00320 & 2.63470 \\
\hline $\mathrm{C}$ & -5.77390 & 9.37870 & 3.66090 \\
\hline $\mathrm{H}$ & -6.19990 & 9.93240 & 2.82360 \\
\hline $\mathrm{H}$ & -6.56220 & 9.31910 & 4.41260 \\
\hline $\mathrm{H}$ & -4.86880 & 11.07430 & 4.69350 \\
\hline $\mathrm{H}$ & -3.90410 & 10.41500 & 3.40090 \\
\hline C & -3.25040 & 4.50170 & -0.00060 \\
\hline O & -3.14940 & 3.45950 & -0.65100 \\
\hline $\mathrm{N}$ & -2.74630 & 5.67370 & -0.40120 \\
\hline $\mathrm{H}$ & -2.80950 & 6.44910 & 0.24800 \\
\hline C & -1.97280 & 5.89270 & -1.61800 \\
\hline $\mathrm{H}$ & -2.15900 & 5.10650 & -2.35170 \\
\hline $\mathrm{H}$ & -2.29550 & 6.82530 & -2.08140 \\
\hline $\mathrm{H}$ & 0.10100 & 6.04250 & -2.21060 \\
\hline $\mathrm{H}$ & -0.15920 & 5.04220 & -0.78690 \\
\hline $\mathrm{C}$ & -4.25710 & 2.19680 & 4.39160 \\
\hline O & -5.16480 & 1.89160 & 5.16660 \\
\hline $\mathrm{N}$ & -3.09310 & 1.54530 & 4.34150 \\
\hline $\mathrm{H}$ & -2.37480 & 1.89920 & 3.72500 \\
\hline C & -2.75870 & 0.37140 & 5.13460 \\
\hline $\mathrm{H}$ & -2.80370 & 0.61540 & 6.19620 \\
\hline $\mathrm{H}$ & -3.49210 & -0.41710 & 4.95720 \\
\hline C & -1.35860 & -0.12480 & 4.76310 \\
\hline $\mathrm{H}$ & -1.34200 & -0.44290 & 3.71900 \\
\hline $\mathrm{H}$ & -3.23750 & 2.52240 & 1.85520 \\
\hline C & -0.47750 & 5.95770 & -1.28920 \\
\hline O & -0.24500 & 7.09270 & -0.46720 \\
\hline C & 1.10770 & 7.18830 & -0.04160 \\
\hline $\mathrm{H}$ & 1.77040 & 7.19130 & -0.90860 \\
\hline $\mathrm{H}$ & 1.38130 & 6.33280 & 0.57610 \\
\hline C & 1.28530 & 8.49550 & 0.73580 \\
\hline $\mathrm{H}$ & 2.33930 & 8.65800 & 0.96570 \\
\hline $\mathrm{H}$ & 0.95280 & 9.33110 & 0.11790 \\
\hline O & 0.54470 & 8.42330 & 1.94800 \\
\hline C & 0.44700 & 9.66740 & 2.63000 \\
\hline $\mathrm{H}$ & 0.85830 & 9.54660 & 3.63260 \\
\hline $\mathrm{H}$ & 1.03390 & 10.45700 & 2.15610 \\
\hline C & -1.03470 & 10.06670 & 2.72580 \\
\hline $\mathrm{H}$ & -1.56690 & 9.35150 & 3.35430 \\
\hline $\mathrm{H}$ & -1.12480 & 11.02740 & 3.23550 \\
\hline $\mathrm{N}$ & -1.66120 & 10.15230 & 1.40100 \\
\hline C & -2.81780 & 9.66690 & -0.43360 \\
\hline $\mathrm{C}$ & -2.21410 & 10.83790 & -0.63930 \\
\hline $\mathrm{H}$ & -2.27330 & 11.43900 & -1.53320 \\
\hline O & -0.42720 & 0.92750 & 4.97770 \\
\hline C & 0.88780 & 0.57350 & 4.56770 \\
\hline $\mathrm{H}$ & 0.91140 & 0.33310 & 3.50350 \\
\hline $\mathrm{H}$ & 1.20920 & -0.31780 & 5.10920 \\
\hline C & 1.85540 & 1.72320 & 4.87260 \\
\hline $\mathrm{H}$ & 2.87960 & 1.37980 & 4.71820 \\
\hline $\mathrm{H}$ & 1.76960 & 2.02580 & 5.91700 \\
\hline O & 1.58650 & 2.81520 & 4.00370 \\
\hline $\mathrm{C}$ & 2.47690 & 3.90130 & 4.20840 \\
\hline $\mathrm{H}$ & 3.49970 & 3.58120 & 4.00220 \\
\hline $\mathrm{H}$ & 2.44730 & 4.23600 & 5.24700 \\
\hline C & 2.09740 & 5.04590 & 3.25870 \\
\hline $\mathrm{H}$ & 2.10530 & 4.68890 & 2.22780 \\
\hline $\mathrm{H}$ & 2.85870 & 5.82650 & 3.30520 \\
\hline $\mathrm{N}$ & 0.79260 & 5.62200 & 3.60280 \\
\hline $\mathrm{C}$ & -1.38050 & 6.07600 & 3.65120 \\
\hline $\mathrm{C}$ & -0.79720 & 6.82470 & 4.58650 \\
\hline
\end{tabular}


S41

-1.29110
-4.56090
-3.80310
-4.40620
-4.48650
-5.41360
-3.53550
-2.49960
-3.88880
-3.63590
-2.88180
-1.82980
-3.22740
-3.05740
-4.11150
-2.78140
-2.24670
-0.53450
-1.59000
-1.61100
0.61280
1.49310
-0.36450
-0.50660
-2.70680
-3.85500
-0.94350
-0.25770
-2.47230
-2.85310
-1.46130
-0.76200
-1.09620
-3.45590
-2.43030
0.45470

7.52820

10.13800

9.33750

9.17970

10.15430

8.76930

8.25160

8.59450

8.27080

6.92960

5.99120

6.28180

5.96160

4.61930

4.33780

4.68130

3.58750

2.29440

1.90520

1.10430

6.53870

7.02980

5.28580

4.47080

2.74070

2.70470

3.39150

4.04700

9.21160

8.18110

11.16670

12.16370

$-0.99150$

9.14580

6.06750

1.86620

5.23780

4.22510

5.12810

6.40780

6.89160

6.33310

7. 26160

7.26360

8.29390

6.74950

7.49640

7.50520

8.53130

6.83390

6.84830

5.78090

7.48460

8.06070

8.77760

9.50000

4.56930

5. 28120

3.00730

2. 09280

8. 42140

8.87210

7.22440

6.43760

0.88660

1. 45040

0.54220

0.74370

5.37210

$-1.12990$

3. 41260

8.09590 
Computational Methods. The conformational ensembles generated in this study were calculated using the MacroModel V9.1 suite of software programs running on 3.2 GHz Athlons under the Red Hat 9 operating system. The Low Mode (LM) search method was used in a 1:1 combination with the Monte Carlo (MC) search method to explore the potential energy surfaces of $4-6$. Each MC conformational search step varied a random number of torsional degrees of freedom between a minimum of two and a maximum of $\mathrm{N}$, where $\mathrm{N}$ is the total number of variable torsion angles in each molecule as shown below. Conformational searches were run with amides constrained to the $E$ rotamer as preliminary results with and without this constraint were indistinguishable. LM frequencies corresponding to the 10 lowest eigenvectors were explored. The total traveling distance for each step was selected randomly between $3 \AA$ and $6 \AA$. Interconversion of ring structures was enabled using the ring-opening method of Still using the wavy bonds as indicated below. Starting structure chirality was preserved throughout the conformational searching. Searches were run in multiple blocks of 5000 LM:MC steps until they had reached convergence. Convergence was judged by monitoring the 1) energy of the most stable structure, 2) number of times this structure was visited and 3) number of unique conformations found within a user-controlled energetic window above the lowest energy structure. Unique conformations were determined by superimposition of all heavy (non-hydrogen) atoms as well as reflection and/or rotation of the atom-numbering scheme. Structures were considered to be duplicates and rejected if the maximum interatomic distance was $0.25 \AA$ or less following optimal RMS superposition. Structures found in previous searches were used to seed subsequent searches. Searches utilized the usage-directed structure selection method that identifies the least used structure from among all known conformations and then uses this structure as the starting point for each new search. This insures that a variety of different starting structures from different regions of the potential energy surface are used to begin each new block of steps. During the conformational search all structures were subjected to 500 steps of the Truncated Newton Conjugate Gradient (TNCG) minimization method to within a derivative convergence criterion of $0.01 \mathrm{~kJ}^{-1} \mathrm{~mol}^{-1}$. All final ensembles were subjected to further TNCG minimization after the search was completed in order to obtain fully minimized structures. 


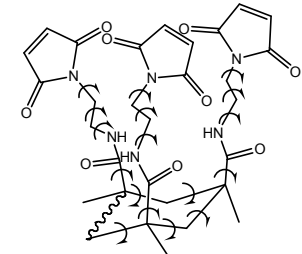

$4 a$
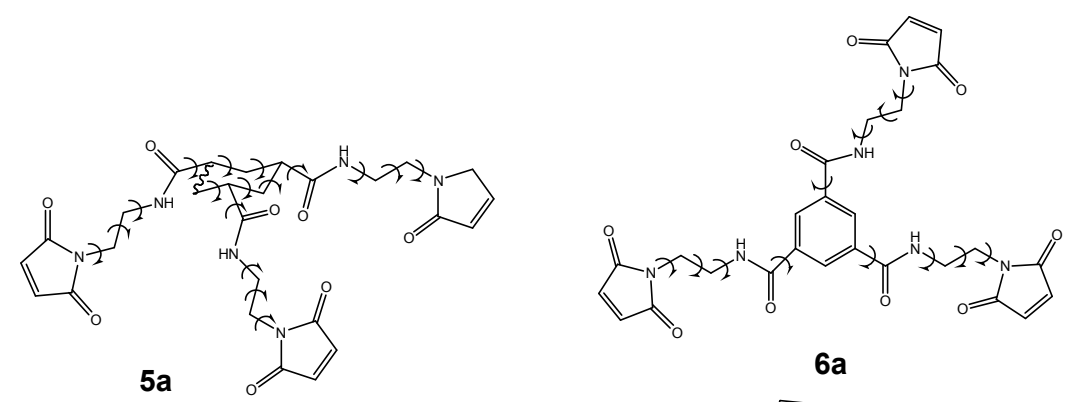

$6 a$
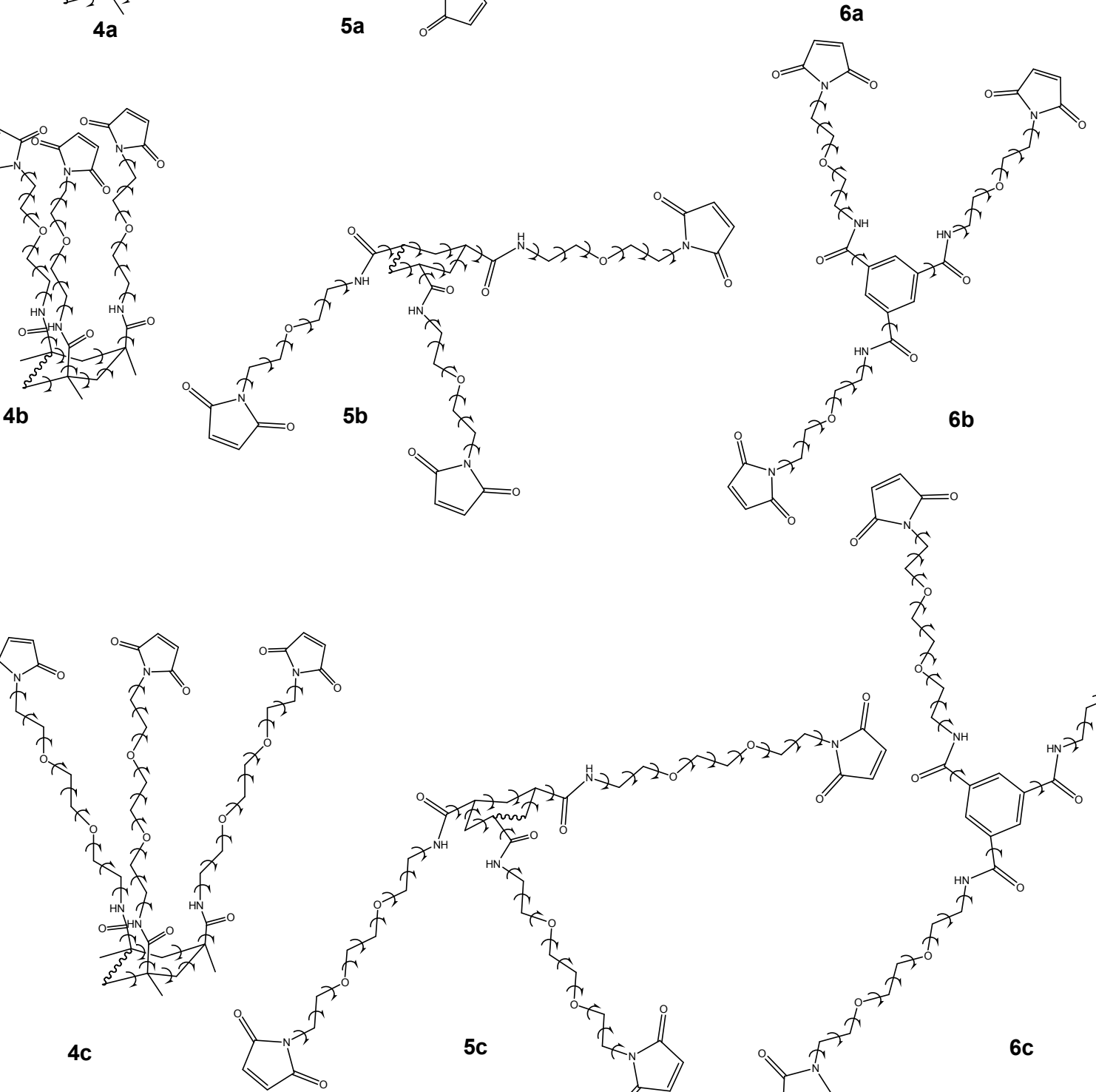

$5 b$
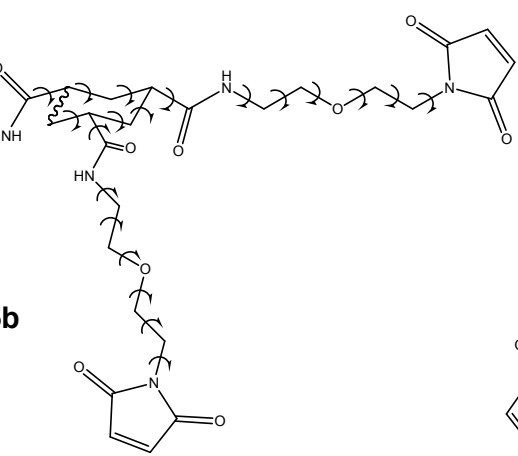

$$
\text { 4b }
$$
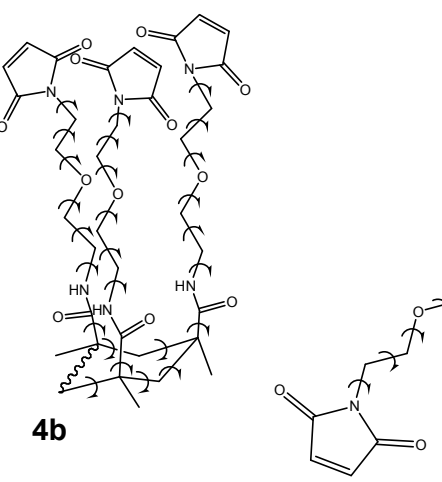

त
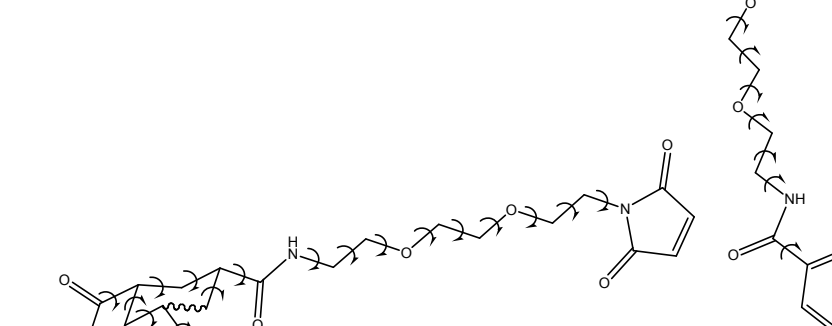

$5 c$
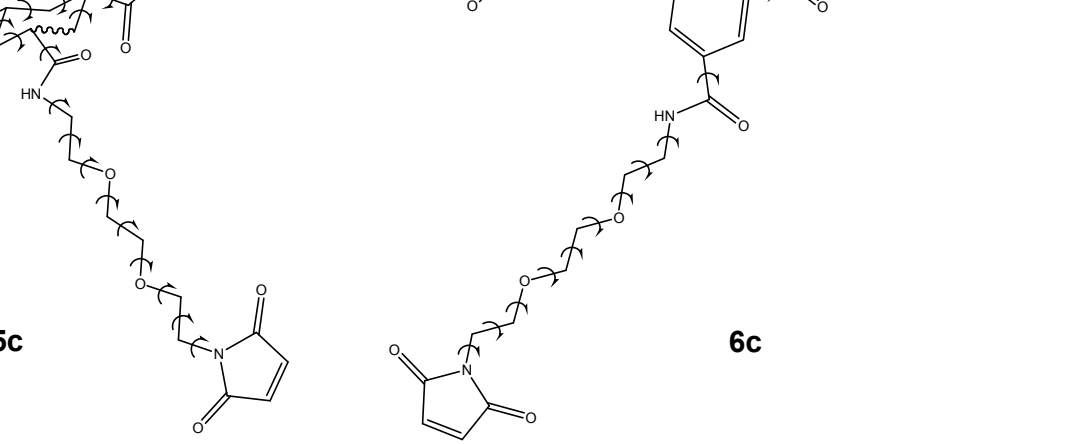

Degrees of freedom varied during the conformational searches of $\mathbf{4}-\mathbf{6}$. Arrows represent torsions that were allowed to vary and the wavy lines indicate the bonds used to open rings for conformational interconversions.

Clustering Ensembles. XCluster calculates the pairwise distance between each structure, in either torsional or Cartesian space, and partitions the conformations into geometrically similar subsets in an agglomerative, hierarchical fashion. The process begins with every structure as the only member of its own cluster. Individual structures are then grouped into clusters using the shortest distance between points as the threshold distance. At each clustering level the next shortest distance is used to form new, agglomerative clusters, with later clusters formed from groupings of earlier clusters. This 
process continues until all structures are a member of the same final cluster. The goal is to find the clustering level at which the distance between the members of each cluster is much smaller than the distance between clusters; i.e., the minimum separation ratio. Separation ratios greater than 2 that occur at high clustering level indicate significant clustering.

XYZ coordinates from lowest energy structure (relative energy $=0.00 \mathrm{kcal} / \mathrm{mol}$; frequency analysis was not performed) found on the B3LYP/6-31G**-SCRF(water) surface obtained using Jaguar V6.5 (input structure below)

\begin{tabular}{|c|c|c|c|}
\hline $\mathrm{C}$ & 7.70730 & 1.64780 & -3.87730 \\
\hline $\mathrm{C}$ & 7.77420 & 0.47010 & -2.87490 \\
\hline $\mathrm{C}$ & 8.20600 & -0.91670 & -3.46970 \\
\hline $\mathrm{C}$ & 8.79440 & -0.75690 & -4.90630 \\
\hline $\mathrm{C}$ & 7.93110 & 0.02910 & -5.92990 \\
\hline $\mathrm{C}$ & 7.10690 & 1.13320 & -5.21030 \\
\hline $\mathrm{H}$ & 6.77040 & 0.35090 & -2.45450 \\
\hline $\mathrm{H}$ & 8.41630 & 0.75800 & -2.04120 \\
\hline $\mathrm{H}$ & 9.74950 & -0.24100 & -4.79480 \\
\hline $\mathrm{H}$ & 9.02570 & -1.75590 & -5.29400 \\
\hline $\mathrm{H}$ & 6.11780 & 0.72940 & -4.96490 \\
\hline $\mathrm{H}$ & 6.92850 & 1.94950 & -5.91500 \\
\hline C & 7.00160 & -1.87790 & -3.44170 \\
\hline $\mathrm{H}$ & 7.22810 & -2.82450 & -3.93800 \\
\hline $\mathrm{H}$ & 6.72180 & -2.10110 & -2.40720 \\
\hline $\mathrm{H}$ & 6.13340 & -1.42600 & -3.92660 \\
\hline $\mathrm{C}$ & 6.77820 & 2.73570 & -3.28310 \\
\hline $\mathrm{H}$ & 6.70310 & 3.61110 & -3.93370 \\
\hline $\mathrm{H}$ & 5.77150 & 2.32710 & -3.14520 \\
\hline $\mathrm{H}$ & 7.14340 & 3.07200 & -2.30690 \\
\hline C & 6.96460 & -0.89730 & -6.71390 \\
\hline $\mathrm{H}$ & 6.27140 & -1.40670 & -6.04150 \\
\hline $\mathrm{H}$ & 6.37600 & -0.30990 & -7.42540 \\
\hline $\mathrm{H}$ & 7.51720 & -1.65790 & -7.27490 \\
\hline C & 11.54740 & -1.42260 & -1.63560 \\
\hline $\mathrm{H}$ & 11.19430 & -2.15610 & -0.90700 \\
\hline $\mathrm{H}$ & 12.09040 & -0.64730 & -1.09150 \\
\hline C & 10.90930 & 0.60860 & -8.33980 \\
\hline $\mathrm{H}$ & 10.66890 & 1.65560 & -8.52590 \\
\hline $\mathrm{H}$ & 11.93830 & 0.55590 & -7.97340 \\
\hline C & 9.34270 & -1.58740 & -2.65650 \\
\hline O & 9.31960 & -2.79800 & -2.39460 \\
\hline $\mathrm{C}$ & 8.80830 & 0.67880 & -7.04310 \\
\hline O & 8.37780 & 1.62270 & -7.71680 \\
\hline $\mathrm{N}$ & 10.39570 & -0.81740 & -2.29070 \\
\hline $\mathrm{H}$ & 10.41800 & 0.16810 & -2.55730 \\
\hline $\mathrm{N}$ & 10.01540 & 0.11340 & -7.30180 \\
\hline $\mathrm{H}$ & 10.35930 & -0.64650 & -6.72790 \\
\hline $\mathrm{C}$ & 10.83310 & -0.18170 & -9.65490 \\
\hline $\mathrm{H}$ & 11.57030 & 0.21560 & -10.35920 \\
\hline $\mathrm{H}$ & 11.05250 & -1.23890 & -9.48940 \\
\hline $\mathrm{C}$ & 12.49420 & -2.14680 & -2.60720 \\
\hline $\mathrm{H}$ & 11.93680 & -2.85520 & -3.22170 \\
\hline $\mathrm{H}$ & 13.24640 & -2.70090 & -2.03900 \\
\hline $\mathrm{N}$ & 13.19990 & -1.24000 & -3.50960 \\
\hline C & 13.92530 & -0.01280 & -5.34210 \\
\hline $\mathrm{C}$ & 14.74760 & 0.28780 & -4.33440 \\
\hline $\mathrm{H}$ & 15.59500 & 0.96110 & -4.31210 \\
\hline $\mathrm{N}$ & 9.51550 & -0.11640 & -10.27420 \\
\hline $\mathrm{C}$ & 7.61290 & 0.64550 & -11.36660 \\
\hline $\mathrm{C}$ & 7.35660 & -0.60500 & -10.97160 \\
\hline $\mathrm{H}$ & 6.46220 & -1.19970 & -11.10480 \\
\hline $\mathrm{C}$ & 14.31510 & -0.49310 & -3.13070 \\
\hline
\end{tabular}




$\begin{array}{rrrr}\mathrm{O} & 14.81210 & -0.50420 & -2.01950 \\ \mathrm{C} & 12.90800 & -1.00280 & -4.84850 \\ \mathrm{O} & 11.99620 & -1.51590 & -5.47850 \\ \mathrm{C} & 9.00530 & 0.99890 & -10.93500 \\ \mathrm{O} & 9.61220 & 2.03800 & -11.12430 \\ \mathrm{C} & 8.56710 & -1.13470 & -10.25780 \\ \mathrm{O} & 8.72180 & -2.23520 & -9.75640 \\ \mathrm{C} & 10.38940 & 4.00210 & -5.33550 \\ \mathrm{H} & 11.24320 & 3.41300 & -4.99770 \\ \mathrm{H} & 10.45020 & 4.11130 & -6.42260 \\ \mathrm{C} & 10.44330 & 5.40440 & -4.70810 \\ \mathrm{H} & 11.33510 & 5.92590 & -5.07120 \\ \mathrm{H} & 9.56580 & 5.99230 & -4.98840 \\ \mathrm{~N} & 10.48710 & 5.37990 & -3.25160 \\ \mathrm{C} & 11.21510 & 5.12240 & -1.05980 \\ \mathrm{C} & 9.92710 & 5.47340 & -0.99990 \\ \mathrm{H} & 9.29330 & 5.62660 & -0.13530 \\ \mathrm{C} & 9.10680 & 2.29470 & -4.06410 \\ \mathrm{O} & 10.09390 & 1.96940 & -3.38390 \\ \mathrm{~N} & 9.17740 & 3.26510 & -5.00600 \\ \mathrm{H} & 8.35810 & 3.46020 & -5.56200 \\ \mathrm{C} & 9.41750 & 5.65020 & -2.40170 \\ \mathrm{O} & 8.29670 & 5.97760 & -2.75410 \\ \mathrm{C} & 11.61820 & 5.05090 & -2.50420 \\ \mathrm{O} & 12.71050 & 4.78380 & -2.97290 \\ \mathrm{H} & 13.93770 & 0.34850 & -6.36230 \\ \mathrm{H} & 6.98370 & 1.34180 & -11.90690 \\ \mathrm{H} & 11.91120 & 4.91240 & -0.25720 \\ & & & \end{array}$

Input file corresponding to the lowest energy structure found on the B3LYP/6$31 \mathrm{G} * \star-\mathrm{SCRF}$ (water) surface obtained using Jaguar V6.5

$\begin{array}{llll}\text { MAEFILE }: \text { mm2_second.mae } & & \\ \text { \&gen } & & & \\ \text { isolv=2 } & & & \\ \text { basis=6-31G** } & & & \\ \text { igeopt=1 } & & & \\ \text { dftname=B3LYP } & & & \\ \text { \& } & & & \\ \text { entry_name: inhib1_ax_mm2_25-out.2 } & \\ \text { \&zmat } & & & \\ \text { C1 } & 7.6041870000000 & 1.4702180000000 & -4.0970610000000 \\ \text { C2 } & 7.6811600000000 & 0.4420320000000 & -2.9418540000000 \\ \text { C3 } & 8.2141880000000 & -0.9572080000000 & -3.3437770000000 \\ \text { C4 } & 8.8198460000000 & -0.9722490000000 & -4.7672010000000 \\ \text { C5 } & 7.9852420000000 & -0.3463390000000 & -5.9075690000000 \\ \text { C6 } & 7.0489350000000 & 0.7466760000000 & -5.3410990000000 \\ \text { H7 } & 6.6547350000000 & 0.3123600000000 & -2.5286770000000 \\ \text { H8 } & 8.2362400000000 & 0.8749680000000 & -2.0783240000000 \\ \text { H9 } & 9.7758330000000 & -0.4076960000000 & -4.7205060000000 \\ \text { H10 } & 9.1122080000000 & -2.0152330000000 & -5.0304410000000 \\ \text { H11 } & 6.0883290000000 & 0.2730330000000 & -5.0353770000000 \\ \text { H12 } & 6.7403930000000 & 1.4724420000000 & -6.1273040000000 \\ \text { C13 } & 7.0728790000000 & -1.9964620000000 & -3.2381010000000 \\ \text { H14 } & 7.3859400000000 & -3.0013170000000 & -3.6040010000000 \\ \text { H15 } & 6.7215790000000 & -2.1144580000000 & -2.1868850000000 \\ \text { H16 } & 6.1781610000000 & -1.6983900000000 & -3.8263620000000 \\ \text { C17 } & 6.6556200000000 & 2.6158510000000 & -3.6813410000000 \\ \text { H18 } & 6.5690980000000 & 3.3982870000000 & -4.4691350000000 \\ \text { H19 } & 5.6239370000000 & 2.2434210000000 & -3.4865370000000 \\ \text { H20 } & 7.0116330000000 & 3.1128680000000 & -2.7499040000000 \\ \text { C21 } & 7.1590670000000 & -1.4096890000000 & -6.6632860000000 \\ \text { H22 } & 6.4441450000000 & -1.9441960000000 & -6.0012890000000 \\ \text { H23 } & 6.5589880000000 & -0.9441020000000 & -7.4788400000000 \\ \text { H24 } & 7.8079020000000 & -2.1883030000000 & -7.1259340000000\end{array}$




\section{S46}

\begin{tabular}{|c|c|c|c|}
\hline $\mathrm{C} 25$ & 11.5515570000000 & -0.9222430000000 & -1.3411520000000 \\
\hline H2 6 & 11.2404570000000 & -1.6148430000000 & -0.5242220000000 \\
\hline 27 & 11.9136130000000 & 0.0041760000000 & -0.8373010000000 \\
\hline 28 & 10.9949610000000 & 0.0718710000000 & -8.3448580000000 \\
\hline 29 & 11.1092860000000 & 1.1738850000000 & -8.2156730000000 \\
\hline & 11.9919370000000 & -0.3667100000000 & -8.1051780000000 \\
\hline & 9.3353890000000 & -1.3981260000000 & -2.3770290000000 \\
\hline & 9.3126630000000 & -2.4798960000000 & -1.8136690000000 \\
\hline & 8.9150720000000 & 0.2411320000000 & -6.9893510000000 \\
\hline & 8.6577370000000 & 1.2897320000000 & -7.555697000000 \\
\hline & 10.4131550000000 & -0.5422930000000 & -2.176228000000 \\
\hline & 10.4040010000000 & 0.3437900000000 & -2.68675100000 \\
\hline 37 & 10.0520550000000 & -0.4651890000000 & -7.363162000000 \\
\hline & 10.2059510000000 & -1.3811740000000 & -6.936582000000 \\
\hline 3 & 10.6351650000000 & -0.2789430000000 & -9.798528000000 \\
\hline 40 & 11.4469820000000 & 0.0617970000000 & -10.483959000000 \\
\hline & 10.5751580000000 & -1.3870100000000 & -9.909703000000 \\
\hline 42 & 12.6913780000000 & -1.5446500000000 & -2.165832000000 \\
\hline 43 & 12.3369860000000 & -2.4963140000000 & -2.626790000000 \\
\hline 4 & 13.5417200000000 & -1.8183400000000 & -1.4973370000000 \\
\hline 4 & 13.1618880000000 & -0.6431530000000 & -3.18972200 \\
\hline 46 & 13.5241990000000 & 0.4034960000000 & -5.191745000000 \\
\hline C4 & 14.2340680000000 & 1.0658740000000 & -4.268 \\
\hline $4 \xi$ & 14.8617660000000 & 1.9494720000000 & -4.46 \\
\hline 4 & 9.3941640000000 & 0.3334330000000 & -10.203203000000 \\
\hline C5 & 1660000000 & 1.8629380000000 & -10.874 \\
\hline . & 7.1915410000000 & 0.6980420000000 & -10.70 \\
\hline 52 & 6.1116100000000 & 0.5405030000000 & -10.8559360000000 \\
\hline 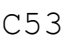 & 14.0178610000000 & 0.4073810000000 & -2.9654720000000 \\
\hline 54 & 14.5059540000000 & 0.7327050000000 & -1.8962370000000 \\
\hline 555 & 12.8313880000000 & -0.7136370000000 & -4.5213520000000 \\
\hline 056 & 12.1092270000000 & -1.5349920000000 & -5.0602240000000 \\
\hline C5 5 & 9.2585560000000 & 1.6515550000000 & -10.5653770000000 \\
\hline 058 & 10.1441180000000 & 2.4885100000000 & -10.6256620000000 \\
\hline C59 & 8.1821950000000 & -0.3090990000000 & -10.27 \\
\hline 060 & 7.9573080000000 & -1.4838040000000 & -10.0360870000000 \\
\hline C61 & 10.4223960000000 & 3.5755730000000 & -5.7504430000000 \\
\hline $\mathrm{H} 62$ & 11.2452350000 & 2.845432000 & -5.56 \\
\hline H6 & 10.4283840000000 & 3.7652660000000 & -6.84 \\
\hline C6 & 10.6803100000000 & 4.8976490000000 & -5.007 \\
\hline H6 & 11.6444200000 & 5.34 & -5.346 \\
\hline H6 & 9.8822390000000 & 5.63246 & -5.266 \\
\hline N67 & 10.7302690000000 & 4.6942270000000 & -3.5808710000000 \\
\hline C68 & 11.4211410000000 & 4.0794500000000 & -1.4877580000000 \\
\hline C69 & 10.1732110000000 & 4.5531600000000 & 90000000 \\
\hline H7O & 9.6093800000000 & 4.6144550000000 & -0.4217790000000 \\
\hline C71 & 8.9751230000000 & 2.1085190000000 & -4.3572160000000 \\
\hline 072 & 9.9319240000000 & 1.8808820000000 & -3.6356380000000 \\
\hline N73 & 9.1255780000000 & 2.9819610000000 & -5.4245090000000 \\
\hline H7 4 & 8.3051610000000 & 3.1610370000000 & -6.0068140000000 \\
\hline C75 & 9.7067080000000 & 4.9641080000000 & -2.7055990000000 \\
\hline 076 & 8.6194600000000 & 5.4475380000000 & -2.9723400000000 \\
\hline C7 7 & 11.8060920000000 & 4.1673760000000 & -2.909334000000 \\
\hline 078 & 12.8741890000000 & 3.8341480000000 & -3.3935010000000 \\
\hline H79 & 13.4827910000000 & 0.6636500000000 & -6.2619120000000 \\
\hline H8O & 7.3544650000000 & 2.8042990000000 & -11.193726000000 \\
\hline 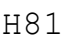 & 12.0351720000000 & 3.6933390000000 & -0.6577480000000 \\
\hline
\end{tabular}




\section{S47}

XYZ coordinates from second lowest energy structure (relative energy $=2.22$ $\mathrm{kcal} / \mathrm{mol}$; frequency analysis was not performed)found on the B3LYP/6-31G**SCRF (water) surface obtained using Jaguar V6.5 (input structure below)

\begin{tabular}{|c|c|c|c|}
\hline $\mathrm{C}$ & 0.34410 & -3.65610 & 1.87330 \\
\hline $\mathrm{C}$ & 1.89210 & -3.62820 & 1.78580 \\
\hline $\mathrm{C}$ & 2.53690 & -3.21340 & 0.43630 \\
\hline $\mathrm{C}$ & 1.85990 & -3.99410 & -0.72040 \\
\hline $\mathrm{C}$ & 0.30870 & -4.04250 & -0.75660 \\
\hline $\mathrm{C}$ & -0.22800 & -4.41920 & 0.64850 \\
\hline $\mathrm{C}$ & 4.03470 & -3.62910 & 0.47350 \\
\hline $\mathrm{C}$ & -0.04240 & -4.44740 & 3.15440 \\
\hline $\mathrm{C}$ & -0.11200 & -5.16950 & -1.74170 \\
\hline $\mathrm{C}$ & 2.41160 & 0.35780 & -1.03540 \\
\hline $\mathrm{C}$ & -1.94590 & -0.99670 & -1.51630 \\
\hline $\mathrm{C}$ & 2.59650 & -1.67130 & 0.31260 \\
\hline 0 & 2.92060 & -0.98730 & 1.30570 \\
\hline $\mathrm{C}$ & -0.26140 & -2.76160 & -1.41310 \\
\hline 0 & 0.29860 & -2.28820 & -2.42330 \\
\hline $\mathrm{N}$ & 2.38970 & -1.09650 & -0.88280 \\
\hline $\mathrm{H}$ & 1.97150 & -1.64020 & -1.63380 \\
\hline $\mathrm{N}$ & -1.40250 & -2.22960 & -0.94510 \\
\hline $\mathrm{H}$ & -1.75120 & -2.51720 & -0.03390 \\
\hline $\mathrm{C}$ & -3.14330 & -0.51970 & -0.68210 \\
\hline $\mathrm{C}$ & 2.01300 & 0.73370 & -2.46940 \\
\hline $\mathrm{N}$ & 1.72600 & 2.15580 & -2.58390 \\
\hline $\mathrm{C}$ & 1.93570 & 4.45630 & -2.79730 \\
\hline $\mathrm{C}$ & 0.63670 & 4.20640 & -2.61220 \\
\hline $\mathrm{N}$ & -3.51890 & 0.84450 & -1.02500 \\
\hline $\mathrm{C}$ & -4.41640 & 2.70420 & -2.08400 \\
\hline $\mathrm{C}$ & -3.68770 & 3.16030 & -1.06160 \\
\hline $\mathrm{C}$ & 0.45310 & 2.72360 & -2.47100 \\
\hline O & -0.58200 & 2.10340 & -2.31810 \\
\hline $\mathrm{C}$ & 2.67600 & 3.15160 & -2.77420 \\
\hline O & 3.87470 & 2.96270 & -2.89890 \\
\hline $\mathrm{C}$ & -4.32250 & 1.20690 & -2.09980 \\
\hline O & -4.84000 & 0.42800 & -2.88290 \\
\hline $\mathrm{C}$ & -3.08670 & 1.98710 & -0.34430 \\
\hline O & -2.38550 & 1.99190 & 0.65000 \\
\hline $\mathrm{C}$ & -0.32580 & 0.07640 & 2.62160 \\
\hline $\mathrm{C}$ & 0.73240 & 1.13790 & 2.95450 \\
\hline $\mathrm{N}$ & 0.19500 & 2.48370 & 2.82040 \\
\hline $\mathrm{C}$ & -0.42100 & 4.55600 & 1.97370 \\
\hline $\mathrm{C}$ & -0.91400 & 4.48320 & 3.21310 \\
\hline $\mathrm{C}$ & -0.38090 & -2.30040 & 2.05640 \\
\hline O & -1.61870 & -2.24070 & 1.90520 \\
\hline $\mathrm{N}$ & 0.31660 & -1.22830 & 2.46490 \\
\hline $\mathrm{H}$ & 1.33290 & -1.24850 & 2.42800 \\
\hline $\mathrm{C}$ & -0.54480 & 3.14900 & 3.79050 \\
\hline O & -0.81820 & 2.70320 & 4.89250 \\
\hline $\mathrm{C}$ & 0.30870 & 3.27890 & 1.67590 \\
\hline 0 & 0.91380 & 2.97600 & 0.66480 \\
\hline $\mathrm{H}$ & 2.32260 & -3.03310 & 2.59690 \\
\hline $\mathrm{H}$ & 2.21900 & -4.65810 & 1.97560 \\
\hline $\mathrm{H}$ & 2.24000 & -3.66690 & -1.69300 \\
\hline $\mathrm{H}$ & 2.19390 & -5.03340 & -0.61250 \\
\hline $\mathrm{H}$ & 0.03700 & -5.47360 & 0.79540 \\
\hline $\mathrm{H}$ & -1.32150 & -4.38820 & 0.67470 \\
\hline $\mathrm{H}$ & 4.53700 & -3.36420 & -0.46320 \\
\hline $\mathrm{H}$ & 4.55400 & -3.12760 & 1.29460 \\
\hline $\mathrm{H}$ & 4.12230 & -4.71130 & 0.61490 \\
\hline $\mathrm{H}$ & -1.12910 & -4.52030 & 3.24990 \\
\hline $\mathrm{H}$ & 0.37250 & -5.45980 & 3.11210 \\
\hline 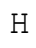 & 0.34880 & -3.95390 & 4.05070 \\
\hline
\end{tabular}




$\begin{array}{lr}\mathrm{H} & -1.20280 \\ \mathrm{H} & 0.26850 \\ \mathrm{H} & 0.28810 \\ \mathrm{H} & 1.71630 \\ \mathrm{H} & 3.41010 \\ \mathrm{H} & -1.17770 \\ \mathrm{H} & -2.24860 \\ \mathrm{H} & -4.01320 \\ \mathrm{H} & -2.88830 \\ \mathrm{H} & 2.80480 \\ \mathrm{H} & 1.10720 \\ \mathrm{H} & 2.44250 \\ \mathrm{H} & -0.19610 \\ \mathrm{H} & -4.99620 \\ \mathrm{H} & -3.52350 \\ \mathrm{H} & -1.08040 \\ \mathrm{H} & -0.84560 \\ \mathrm{H} & 1.10800 \\ \mathrm{H} & 1.57660 \\ \mathrm{H} & -0.48370 \\ \mathrm{H} & -1.48960\end{array}$

$$
\begin{array}{r}
-5.25230 \\
-4.96540 \\
-6.13200 \\
0.82290 \\
0.74280 \\
-0.21740 \\
-1.16710 \\
-1.16550 \\
-0.52620 \\
0.48210 \\
0.19710 \\
5.40220 \\
4.89650 \\
3.25190 \\
4.17940 \\
0.02840 \\
0.35570 \\
1.01610 \\
1.05660 \\
5.36390 \\
5.21120
\end{array}
$$

S48

$-1.79750$

$-2.74620$

$-1.40640$

$-0.33000$

$-0.80280$

$-1.53060$

$-2.55480$

$-0.83010$

0.37990

$-3.18030$

$-2.76000$

$-2.94110$

$-2.57280$

$-2.81620$

$-0.73660$

3.41380

1.70020

3.97420

2. 26620

1.25630

3.77030

Input file corresponding to the second lowest energy structure found on the B3LYP/6-31G**-SCRF (water) surface obtained using Jaguar V6.5

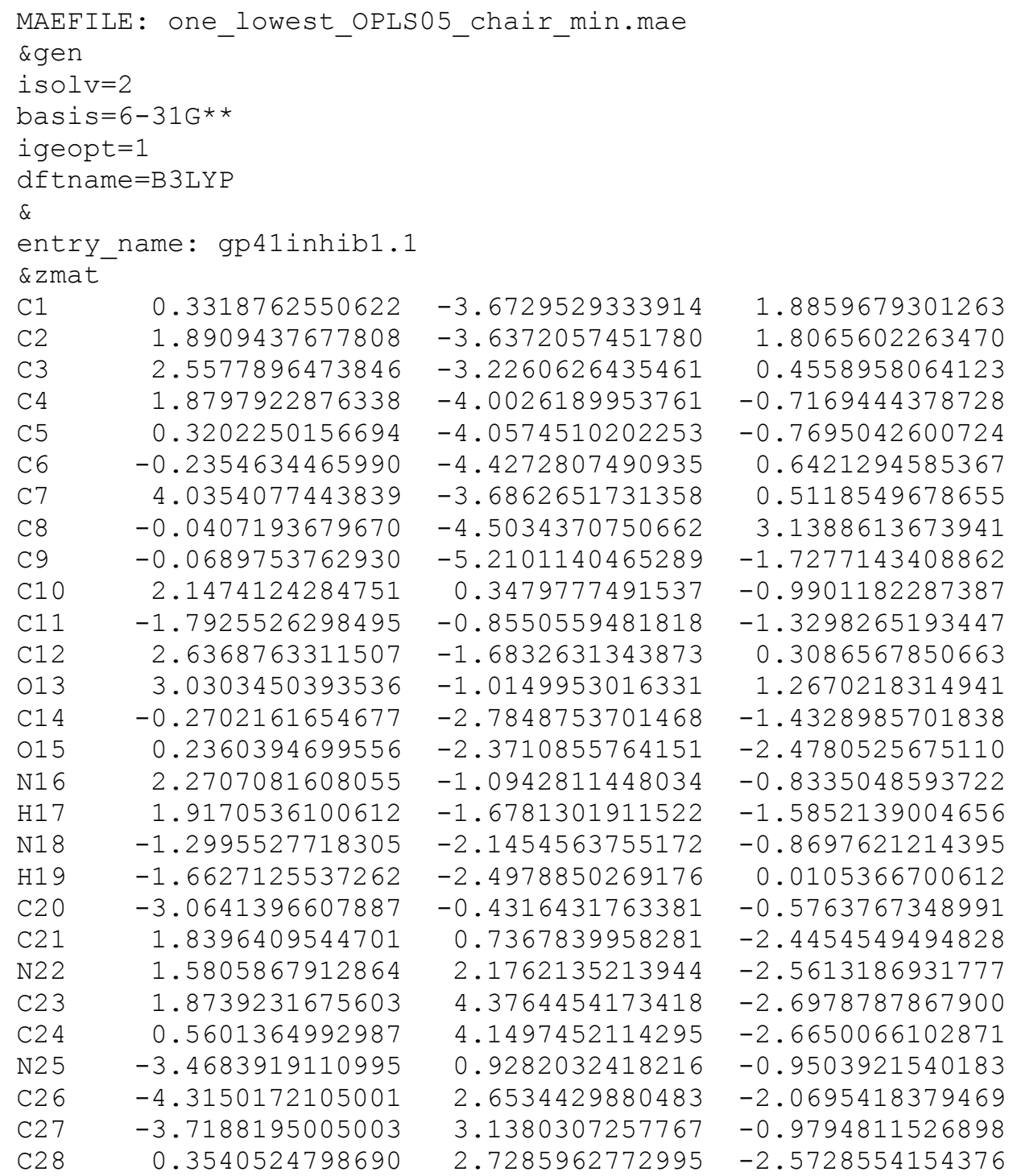

$-3.6729529333914$ $-3.6372057451780$ $-3.2260626435461$ $-4.0026189953761$ $-4.0574510202253$ $-4.4272807490935$ $-3.6862651731358$ $-4.5034370750662$ $-5.2101140465289$ 0.3479777491537 $-0.8550559481818$ $-1.6832631343873$ $-1.0149953016331$ $-2.7848753701468$ $-2.3710855764151$ $-1.0942811448034$ $-1.6781301911522$ $-2.1454563755172$ $-2.4978850269176$ $-0.4316431763381$

0.7367839958281 2.1762135213944 4. 3764454173418 4.1497452114295 0.9282032418216 2. 6534429880483 3.1380307257767 2. 7285962772995

1.8859679301263

1.8065602263470

0.4558958064123

$-0.7169444378728$

$-0.7695042600724$

0.6421294585367

0.5118549678655

3.1388613673941

$-1.7277143408862$

$-0.9901182287387$

$-1.3298265193447$

0.3086567850663

1. 2670218314941

$-1.4328985701838$

$-2.4780525675110$

$-0.8335048593722$

$-1.5852139004656$

$-0.8697621214395$

0.0105366700612

$-0.5763767348991$

$-2.4454549494828$

$-2.5613186931777$

$-2.6978787867900$

$-2.6650066102871$

$-0.9503921540183$

$-2.0695418379469$

$-0.9794811526898$

$-2.5728554154376$ 


\begin{tabular}{|c|c|c|c|}
\hline 029 & -0.7208144760510 & 2.1258661389810 & -2.5104020703407 \\
\hline C30 & 2.5488854088774 & 3.1070906647550 & -2.6284156385686 \\
\hline 31 & 3.7651794850554 & 2.8940517563253 & -2.6275381349825 \\
\hline & -4.1586412673032 & 1.2226388225798 & -2.0664139575259 \\
\hline & -4.5650272771824 & 0.4135768156096 & -2.9060728766568 \\
\hline & -3.1621223989860 & 2.0324316822539 & -0.2457487053539 \\
\hline & -2.5266153695779 & 2.0734983146169 & 0.8111174529564 \\
\hline & -0.2444003774570 & 0.1300448568474 & 2.37406328348 \\
\hline & 0.7885664514294 & 1.1828100203988 & 2.80830644289 \\
\hline & 0.2349630841674 & 2.5373334907929 & 2.70208997 \\
\hline & -0.3111916763909 & 4.5560128298233 & 1.94476349 \\
\hline & -0.9068196860231 & 4.3977474793388 & 3.1274241283216 \\
\hline & -0.3806907009776 & -2.3106577243108 & 2.0970668554872 \\
\hline & -1.6104396135106 & -2.2662186088778 & 2.0221951729294 \\
\hline & 0.3315281034040 & -1.2067727828894 & 2.3413880385261 \\
\hline & 1.3441328900339 & -1.2801365553067 & 2.3576901 \\
\hline & -0.5648370046611 & 3.0929356245280 & 3.6295951326821 \\
\hline & -0.9223490212963 & 2.5708592752805 & 4.6899632673149 \\
\hline & 0.4296643009822 & 3.3572809790635 & 1.6535575819629 \\
\hline & 1.1047800926891 & 3.1142274191422 & 0.64973 \\
\hline & 2.2761417262276 & -2.9242395821825 & 2.53553 \\
\hline & 2.2891214437736 & -4.6284855003886 & 2.0231 \\
\hline & 2.1748094875105 & -3.5570211163145 & -1.66694 \\
\hline & 2.1943137373548 & -5.0459656869516 & -0.69236 \\
\hline & -0.0438017660259 & -5.4952918083485 & 0.74565 \\
\hline & -1.3097192986089 & -4.2565884814885 & 0.57185 \\
\hline & 4.5362310103049 & -3.4156473716265 & -0.4176836606049 \\
\hline & 936453334 & -3.199 & 1.34884 \\
\hline & 4.0749904331743 & -4.7675202440222 & 0.6438424 \\
\hline & -1.1255131285980 & -4.5539059450894 & 3.2325384193054 \\
\hline & 0.3622213040579 & -5.511415 & 3.04028 \\
\hline & 0.3785115424201 & -4.0303519495038 & 4.02685 \\
\hline & -1.1549127030093 & -5.2799952552793 & -1.7906169569512 \\
\hline & 0.3399310246354 & -5.0131906685426 & -2.7187323722470 \\
\hline & 0.3337296991515 & -6.1496046906053 & -1.3491 \\
\hline & 1.3382216809939 & 0.7146870075055 & $-0.3585 \varepsilon$ \\
\hline & 3.0817256278347 & 0.8269543904365 & -0.69730 \\
\hline & -1.0284901935165 & -0.0947887986971 & -1.167 \\
\hline & -2.0268577622646 & -0.9130073669798 & -2.392767 \\
\hline & -3.8762663750500 & -1.1168090219972 & -0.8194774628400 \\
\hline & -2.8766560433688 & -0.4575417174057 & 0.4970 \\
\hline & 2.6905750677261 & 0.4830027964032 & -3.077599 \\
\hline & 0.9584449291789 & 0.1945534597175 & -2.788353 \\
\hline & 2.2457945005198 & 5.3880980124963 & -2.766221 \\
\hline & -0.1319469424359 & 4.9777803585444 & -2.7071765619009 \\
\hline & -4.8001021015074 & 3.3243038840373 & -2.7631110 \\
\hline & -3.7214686394853 & 4.2012852748694 & -0.7900537367848 \\
\hline & -1.0782592874414 & 0.1272919175583 & 3.0760403055882 \\
\hline & -0.6110874820908 & 0.3701109922371 & 1.3760608 \\
\hline & 1.0773101534212 & 1.0036790343796 & 3.8439893887864 \\
\hline & 1.6688367432342 & 1.1141067641117 & 2.16916320703 \\
\hline & -0.4401283926700 & 5.4760469303683 & 1.39402 \\
\hline & -1.5184427454886 & 5.1894560549177 & 3.534259 \\
\hline
\end{tabular}


